\title{
Electrical and mechanical characteristics of surface AC dielectric barrier discharge plasma actuators applied to airflow control
}

\author{
Nicolas Benard • Eric Moreau
}

Received: 21 July 2014 / Revised: 6 October 2014 / Accepted: 18 October 2014 / Published online: 5 November 2014

(C) The Author(s) 2014. This article is published with open access at Springerlink.com

\begin{abstract}
The present paper is a wide review on AC surface dielectric barrier discharge (DBD) actuators applied to airflow control. Both electrical and mechanical characteristics of surface DBD are presented and discussed. The first half of the present paper gives the last results concerning typical single plate-to-plate surface DBDs supplied by a sine high voltage. The discharge current, the plasma extension and its morphology are firstly analyzed. Then, time-averaged and time-resolved measurements of the produced electrohydrodynamic force and of the resulting electric wind are commented. The second half of the paper concerns a partial list of approaches having demonstrated a significant modification in the discharge behavior and an increasing of its mechanical performances. Typically, single DBDs can produce mean force and electric wind velocity up to $1 \mathrm{mN} / \mathrm{W}$ and $7 \mathrm{~m} / \mathrm{s}$, respectively. With multi-DBD designs, velocity up to $11 \mathrm{~m} / \mathrm{s}$ has been measured and force up to $350 \mathrm{mN} / \mathrm{m}$.
\end{abstract}

\section{Introduction}

Initially devoted to surface treatment, ozone production or decontamination, weakly ionized gas formed at the surface of a dielectric material emerged as a flow actuator at the end of the 1990s. This new application arose mostly thanks to the pioneer work done at University of Tennessee where the potential of non-thermal surface plasma for producing a thin wall jet was demonstrated (Roth and

N. Benard $\cdot$ E. Moreau $(\bowtie)$

Institut PPRIME, Université de Poitiers (CNRS UPR 3346,

ISAE-ENSMA), Boulevard Marie et Pierre Curie,

BP 30179, 86962 Futuroscope, France

e-mail: eric.moreau@univ-poitiers.fr
Sherman 1998). Then, different groups already having background experiences on corona discharges and their interactions with quiescent or moving flows formed a new highly motivated community that contributes to the dissemination of the advantages and relevancy of non-thermal plasma discharges as an alternative to conventional flow actuators (Moreau 2007; Corke et al. 2009, 2010). Rapidly, the number of publications in journals and conference exponentially grows to finally become a full interdisciplinary research field. The sudden interest for surface dielectric barrier discharge (DBD) energized by AC high voltage for manipulating airflows was initially motivated by the easy implementation of these actuators and a possible retrofitting on existing airfoils. They have the capability to be mounted at the surface of linear or curved objects with a minimal protrusion in the flow. Beside, their location can be changed faster than other active actuators that require a new model for each new position of actuation. The amplitude and frequency of the electrohydrodynamic (EHD) force produced by the surface plasma are directly connected to the driven electrical signal, this being a clear advantage for parametric studies on the sensibility of one flow to well-defined perturbations. Indeed, the EHD force (also referred as EFD force for electro-fluid dynamic) and the resulting produced flow called electric wind or ionic wind are due to electric field that acts on charged species. These charged species are produced by physical phenomena such as ionization, recombination, attachment, detachment and photoionization, which occur at timescale of a few picoseconds (Boeuf et al. 2009a). Subsequently, the produced body force, despite being low-pass filtered by fluid mechanical laws (viscosity, energy exchanges, dissipation) to produce electric wind, has a high bandwidth. Plasma actuators, and more specifically dielectric barrier discharge actuators, have demonstrated their authority to 
manipulate the dynamics of different flows, such as separated flows (Corke and Post 2005; Little and Samimy 2010; McLaughlin et al. 2006; Benard and Moreau 2011; Kelley et al. 2012; Jukes and Choi 2009), developing shear layers (Sosa et al. 2009a; Benard et al. 2008; Thomas et al. 2008) or boundary layer laminar-to-turbulent transitions (Joussot et al. 2010; Grundmann and Tropea 2007; Hanson et al. 2010). In most of these papers, the actuator is used in context of open-loop control, but plasma discharges find a new route in the construction of closed-loop strategies by using DBD (Lombardi et al. 2012; Benard et al. 2010, 2011; Rethmel et al. 2011; Grundmann and Tropea 2008; Kriegseis et al. 2011a). Instead of manipulating a flow dynamic, the actuator can be used as a generator of predefined perturbations easily tuned by the applied electrical signal (Widmann et al. 2012). Again, this original aspect takes its essence when used in closed-loop approach. Recently, new directions emerge such as the use of plasma discharge as flow sensor (Matlis et al. 2008; Hollick et al. 2011) or adaptive optical lens by plasma discharge (Neiswander et al. 2010). A complete list of studies related to plasmaflow interactions for aeronautical applications is no longer possible because the large number of groups in the world working on or using plasma actuators. A part of them focus on the use of plasma discharge for new flow conditions or new aerodynamic configurations, when another part looks at a better insight into the physical aspects of the electromechanical conversion with objective of improving the performances of dielectric barrier discharge plasma actuators. Several new articles are published per month. Most of the time, the presented results are original and compose a real improvement of the physical knowledge and performances of plasma actuators. Sometimes papers simply consist of reproducing experiments that have already been conducted and for which no relevant new results are reported. As mentioned previously, dielectric barrier discharge for flow control is a quickly evolving research field. However, there are few articles that can serve as reference review paper for groups starting experiments with plasma actuators. In the review of Moreau published in (2007), one can find the basic of plasma actuators and a review of experimental flow control investigations. In Corke et al. (2009, 2010), a survey of work preferentially done in the USA is presented. The present paper is an attempt to complete references (Moreau 2007; Corke et al. 2009, 2010) by establishing an up-to-date description of the current knowledge on the EHD force and the resulting electric wind. Only results for actuators operating in quiescent air and producing a tangential electric wind wall jet (i.e., in streamwise forcing) are presented. Moreover, surface DBD supplied by a nanosecond pulse high voltage will not be discussed in the present paper. A review on this topic has been recently published (Adamovich et al. 2012).
The first part of the paper summarizes the main electrical and mechanical characteristics of a typical plate-to-plate linear surface DBD supplied by a sine high voltage. First, the surface discharge is characterized by current measurements, iCCd visualizations and electrical power consumption computations. Secondly, experimental measurements of the time-averaged EHD force and the resulting electric wind are presented. Finally, time-resolved measurements of the actuator mechanical properties are discussed.

The second part of the paper concerns a partial list of approaches having potentiality to improve the performances of surface DBD used in context of in-lab flow control applications. This list is still quite large because flow control improvement largely depends on the baseline flow. First, in the case of low-velocity flow for instance, the key parameter for achieving an effective flow control authority is the maximum mean electric wind velocity. Then, several optimizations that increase the electric wind velocity are presented. Secondly, when the control mechanism relates to dump or amplify natural flow instabilities, optimization of the actuator consists mainly of maximizing the amplitude of the fluctuations caused by the plasma discharge at a specific frequency, the maximum electric wind velocity being not necessarily a key parameter. This can be achieved by modulation of the applied high voltage at an appropriate frequency and duty-cycle. Finally, one needs sometimes to act on a large surface; it is the case of separated flows for which the point of flow detachment moves in a periodic or unsteady manner over a large distance, as along an inclined airfoil for instance. For these types of flow, large-scale actuators are developed. Then, in the last part of this paper, actuators whose geometry consists in DBD assembled in series and DBD based on three electrodes are presented.

\section{Typical single DBD}

The first paper dealing with surface dielectric barrier discharge in air at atmospheric pressure is the one of Masuda and Washizu (1979). Roth used it for the first time for airflow applications in Roth and Sherman (1998). Then, a few research groups in the world started to study DBD plasma actuators and their ability to manipulate airflow (Corke and He 2004; Enloe et al. 2004a, b; Pons et al. 2004, 2005). In this part, the electrical and mechanical characteristics of a typical single plate-to-plate surface dielectric barrier discharge actuator energized by a periodic sine waveform high voltage are summarized. Only experimental results are presented.

\subsection{Actuator design}

Single DBD plasma actuator is usually composed of two flat electrodes flush mounted on both sides of a dielectric, 

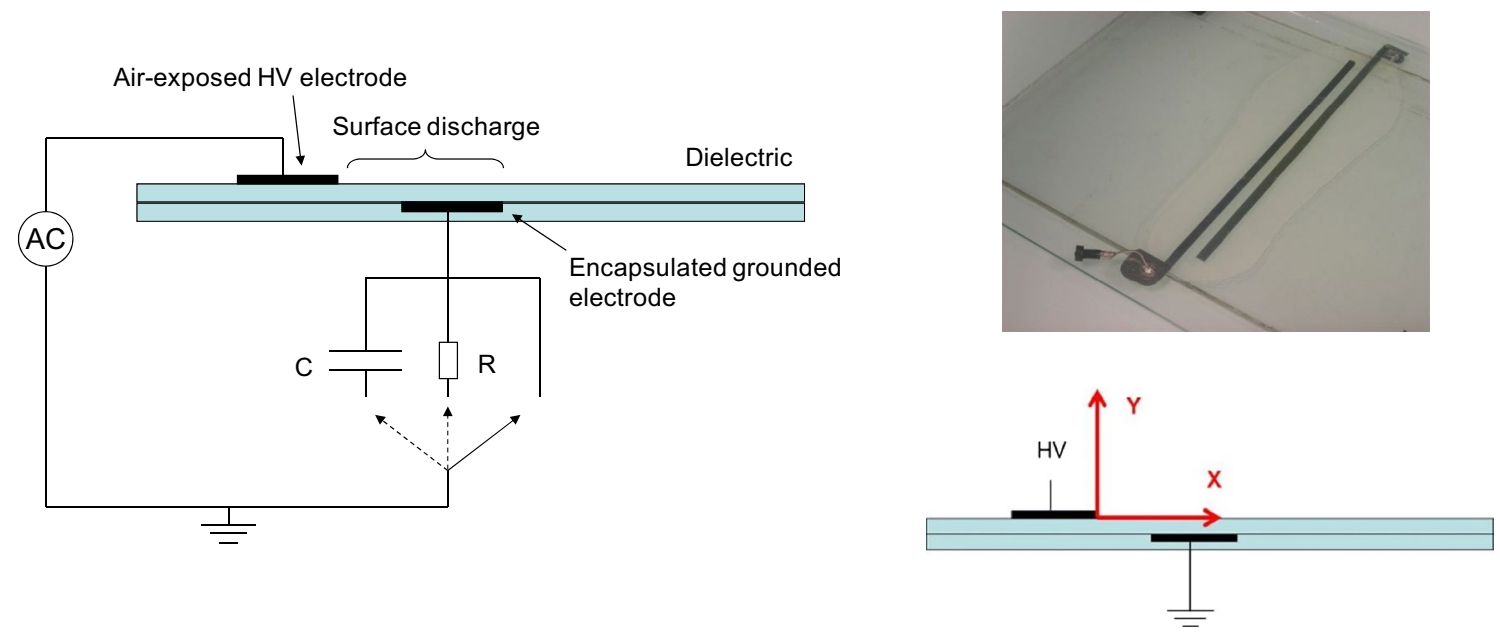

Fig. 1 Typical single dielectric barrier discharge plasma actuator design

as shown in Fig. 1 (in the whole paper, the coordinate system $(O, x, y)$ remains similar to Fig. 1). The air-exposed active electrode is connected to an AC high-voltage power supply, and the other one is encapsulated and grounded. Typically, geometrical parameters are as follows: electrode width of a few $\mathrm{mm}$, electrode gap equal to zero or a few $\mathrm{mm}$, and a dielectric in Plexiglas, Kapton, Teflon, glass or ceramics for instance. Its thickness is usually between $0.1 \mathrm{~mm}$ and a few $\mathrm{mm}$. The electrode length (in span wise) depends on the profile on which the actuator is mounted. The active electrode is supplied by a HV transformer (in this case, the frequency and the HV waveform cannot be modified) or a HV amplifier such as a TREK amplifier (Pons et al. 2004). Typical HV magnitudes range from a few $\mathrm{kV}$ to $50 \mathrm{kV}$, depending on the dielectric thickness, for frequencies between $100 \mathrm{~Hz}$ to a few tens of $\mathrm{kHz}$. In these conditions and above the ignition voltage $\mathrm{V}_{0}$, a plasma sheet appears on the air-exposed electrode side of the dielectric, as illustrated in Fig. 2 that presents a top-view photograph with the exposure time of a few seconds (Forte et al. 2006). The surface discharge looks like a quasi-uniform glow, but in fact it is constituted of lots of microdischarges. This point will be clarified in the next section.

\subsection{Discharge current}

In Fig. 3a, one can see the discharge current versus time in the case of a single DBD mounted on a $3 \mathrm{~mm}$-thick PMMA plate energized by a $22 \mathrm{kV}$ ( $44 \mathrm{kV}$ peak-to-peak) high voltage at $1.5 \mathrm{kHz}$. The red curve represents the sine high voltage, the black one is the current during one AC cycle, and the blue one is an envelope of $60 \mathrm{AC}$ cycles. The non-dimensional time $t^{*}=t \times f_{\mathrm{AC}}$ where $f_{\mathrm{AC}}$ is the sine high-voltage frequency. Usually, this current is measured with the help of a non-inductive shunt resistor $R$ (typically

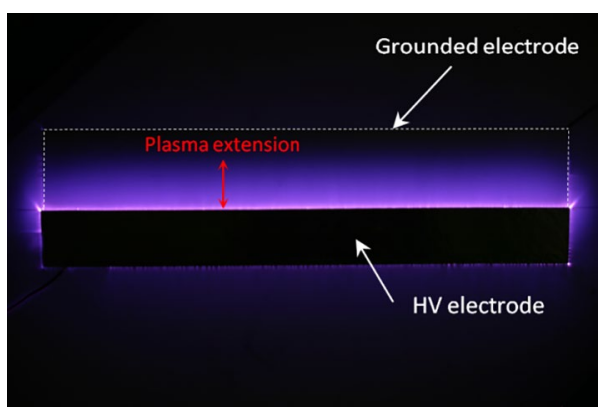

Fig. 2 Photograph of the DBD actuator (top view with exposure time of a few seconds). From Forte et al. (2006)

equal to about $100 \Omega$ ) as shown in Fig. 1. It may also be measured with a high bandwidth coil (current transformer). This current is composed of three components: a capacitive current due to the capacitance of the actuator (even in the absence of discharge), the quasi-synchronous current and lots of current peaks. To plot the discharge current, the capacitive current has been removed in Fig. 3 .

For a plate-to-plate DBD, the discharge current is characterized by two distinct periods of pulses, corresponding to the both discharges occurring during both going cycles (Fig. 3, from Debien et al. 2012a). The positive-going cycle is characterized by high current pulses, up to $250 \mathrm{~mA}$ in Fig. 3a, that are due to streamer propagation. The intensity of these current peaks depends strongly on the power supply characteristics. The glow-like regime occurring during the negative-going cycle results in small pulses up to $10 \mathrm{~mA}$ in magnitude (see the blue curve). Figure $3 \mathrm{~b}$ corresponds to a zoomed view of the discharge current (between -3.5 and $+2 \mathrm{~mA}$ ) in order to visualize more accurately the synchronous current. During the positivegoing cycle, the current is mainly composed of streamers, 


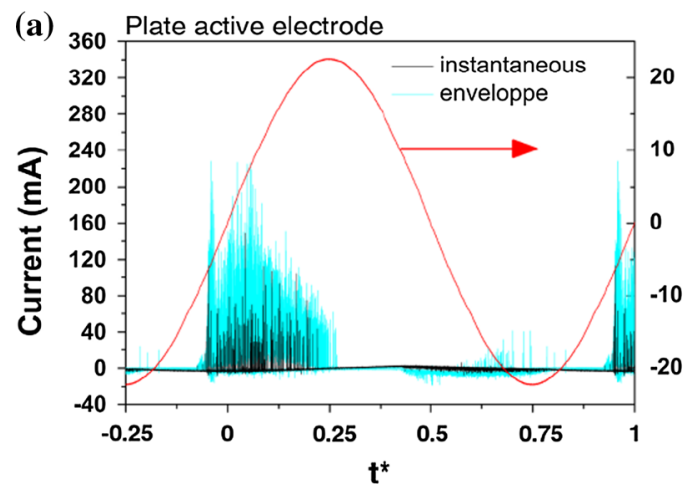

Fig. 3 Typical current versus time for $22 \mathrm{kV}$ and $1.5 \mathrm{kHz}$ : a wide view in the range between -40 and $+360 \mathrm{~mA}$ (instantaneous snapshot of one AC cycle in black and envelope of $60 \mathrm{AC}$ cycles in blue);

and the synchronous current is very small. During the negative-going cycle, one can observe more intense synchronous current, plus small current peaks (up to a few mA). This discharge current analysis conducted for the first time by Pons et al. (2004) highlights that a streamer discharge occurs during the positive-going cycle when a glow-like discharge ignites during the negative-going cycle.

In such conditions, a deeper analysis on the discharge current performed by Laurentie et al. (2009) showed that the number of streamers increases with the applied voltage, the mean streamer duration is equal to $30 \mathrm{~ns}$ and the charge transferred by one streamer is equal to about $35 \mathrm{pC}$, whatever the applied high voltage.

Of course, the time history of the discharge current depends strongly on the geometry of the actuator (electrode shape and size, electrode gap, dielectric thickness for instance), electrical characteristics of the power supply, the environmental conditions (such as humidity, pressure, temperature, ambient gas) and the physicochemical properties of the dielectric material. The geometrical and electrical influence on the produced body force and resulting electric wind will be discussed further in the second part of the paper.

Some authors present current plots with high negative current peaks during the positive-going cycle. These phenomena do not correspond to the plasma physics of surface DBD. They are usually due to parasitic capacitances (bad electrical connections that promote sparks and cable capacitance of at least $50 \mathrm{pF} / \mathrm{m}$ ) and inductive effects (power supply outlet and cable inductance of $1 \mu \mathrm{H} / \mathrm{m}$ ).

\subsection{Plasma extension}

To observe the plasma formation, fast imaging of the ionization process can be conducted. It is the case in Fig. 4 with a 3-mm-thick dielectric. Here, images of the plasma are collected by a fast gateable iCCD camera. The camera

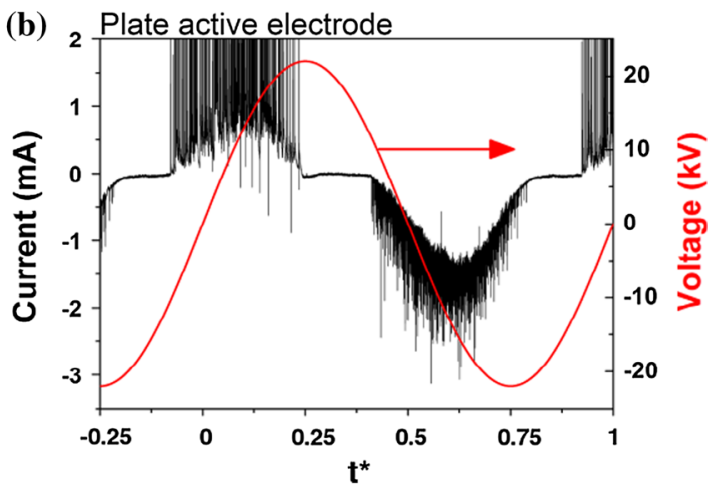

b zoomed view of the same current between $-3.5 \mathrm{~mA}$ and $+2 \mathrm{~mA}$. Note that the non-dimensional time $t^{*}=t / T_{\mathrm{AC}}$. From Debien et al. (2012a)

is placed in front (top view) of the plasma discharge. The field of view $\left(26 \times 26 \mathrm{~mm}^{2}\right.$, with a resolution of about $25 \mu \mathrm{m} / \mathrm{pixel}$ ) is obtained by using a $105-\mathrm{mm}$ macrolens that captures visible and UV wavelengths (Nikkor).

The positive-going cycle is characterized by propagation of streamers that extend from the active electrode up to about $20 \mathrm{~mm}$ (time windows A and B). These filaments are 'stochastically' spread over the surface, with a bright spot zone in contact with the active electrode (corona zone) and an extension from this spot toward the dielectric surface (streamer propagation). These extensions are branched over several filaments with erratic propagation paths. As indicated by the current (see Figs. 3, 4a), the filamentary regime conduces to current peaks of high amplitude with a sparse temporal distribution. During the negativegoing cycle (time windows $\mathrm{C}$ and $\mathrm{D}$ ), diffuse microdischarges occur, as observed in 2008 by Hoskinson et al. (2008a). The surface discharge is then composed of corona regions that enlarge from the spot to the dielectric surface and extend with a plume shape. They extend up to about $10 \mathrm{~mm}$. Moreover, the discharge does not ignite homogeneously along the high-voltage electrode, but it starts from individual spots.

From light emission analysis (time-averaged emission, PMT or iCCD camera), several authors studied the surface discharge morphology. The first one was Enloe et al. (2004a). Orlov (2006) analyzed the plasma extension versus applied high voltage (Fig. 5a) and compared his results with those of Enloe et al. They demonstrate that the maximum extent increases linearly with the voltage amplitude. Another analysis is presented in Forte (2007). More recently, Kriegseis et al. (2011b) confirms this result (Fig. 5b). One can see that in both cases, the plasma extension length is limited to a few mm (typically between 3 and $8 \mathrm{~mm}$ ) because the dielectric thickness was smaller than $1 \mathrm{~mm}$ in both cases. With thicker dielectrics, the plasma can extend up to $20 \mathrm{~mm}$. 


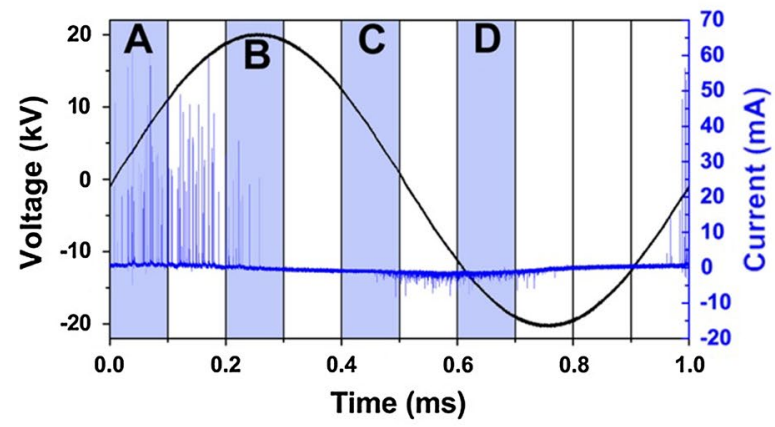

(a)
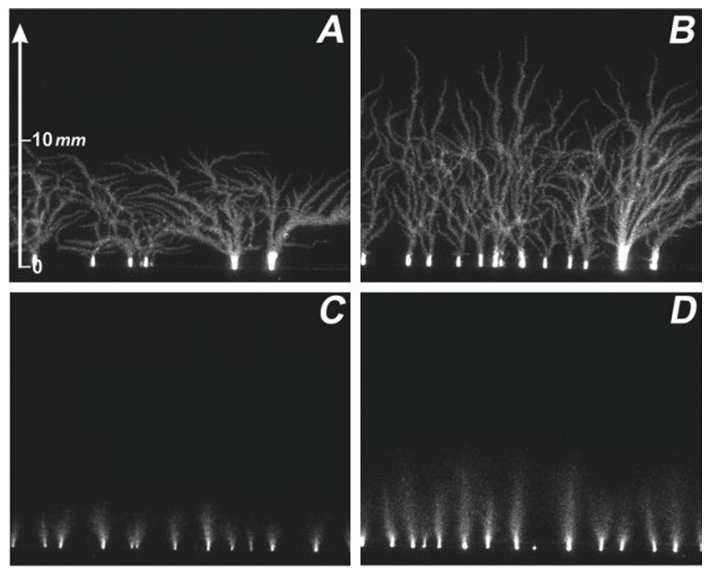

(b)

Fig. 4 Typical current versus time (a) and fast imaging of the developing plasma layer, from top to bottom (images are for a top view and opening gate width of $100 \mu \mathrm{s})$. From Benard and Moreau (2012)

Fig. 5 Plasma extension length versus applied voltage in the case of thin dielectrics. a From Enloe et al. (2004a), b from Kriegseis et al. (2011b)

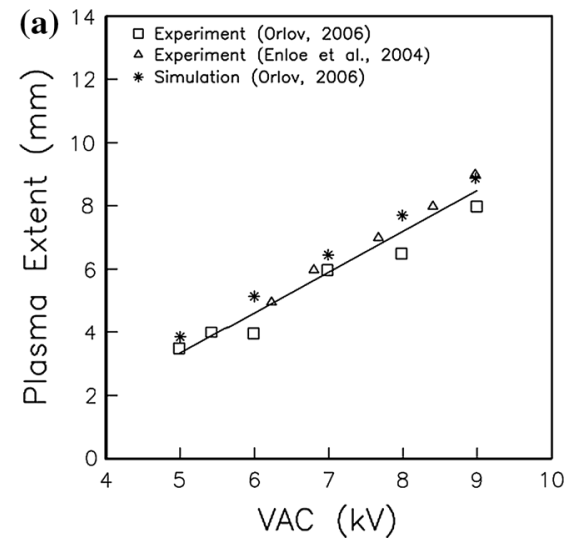

\subsection{Electrical power consumption}

From both voltage and current curves, the time-averaged electrical power consumed by the plasma actuator can be computed with:

$P_{\text {elec }}=\frac{1}{T_{\mathrm{AC}}} \int_{t=0}^{T_{\mathrm{AC}}} v(t) \times i(t) \mathrm{d} t=f_{\mathrm{AC}} \int_{t=0}^{T_{\mathrm{AC}}} v(t) \times i(t) \mathrm{d} t$

where $v(t)$ and $i(t)$ are, respectively, the voltage and the current versus time, $T_{A C}$ is the waveform period, and $f_{\mathrm{AC}}$ is the sine high-voltage frequency. Usually, with this method, the electrical power consumption varies from one to another cycle. Then, several cycles (from 10 to 100) are needed for reaching a converged mean value. Furthermore, data have to be recorded with a high bandwidth oscilloscope (at least $200 \mathrm{MHz}$ up to $1 \mathrm{GHz}$ ) and a high sampling rate (typically a few ns) to be sure that the current peaks are well visualized and sampled. This method is very simple and accurate, but a problem remains: in the presence of high current peaks, such as in Fig. 3, it is difficult to have a good resolution on the synchronous current because its amplitude is at least one order of magnitude smaller than the current peaks.

Another simple method is the Manley's one (Manley 1943; Wagner et al. 2003) used for the first time for surface DBD plasma actuator in 2004 by Pons et al. $(2004,2005)$. This method consists in placing a capacitor $\mathrm{C}$ between the grounded electrode and earth (see Fig. 1), and in plotting the $V-Q$ curve, as illustrated in Fig. 6. The area of this curve corresponds to the energy dissipated by the discharge per period. The curve is covered anticlockwise, and each half part shows two branches corresponding to the presence (A) or not (B) of discharge. Typical energy values ranges from 1 to $20 \mathrm{~mJ}$, and the electrical charge transferred per period is up to a few $\mu \mathrm{C}$. The electrical power is obtained by multiplying this value with the waveform frequency $f_{\mathrm{AC}}$ :

$P_{\text {elec }}=f_{\text {AC }} \int_{\text {cycle }} v(t) \mathrm{d} Q$ 


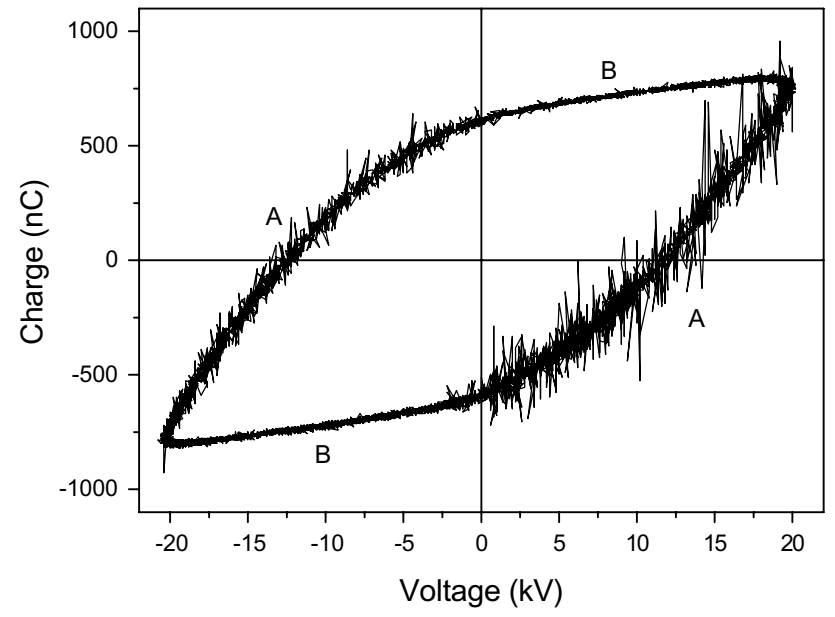

Fig. 6 Charge-voltage curve, usually called «Lissajous loop» $\left(V=20 \mathrm{kV}, f_{\mathrm{AC}}=300 \mathrm{~Hz}\right)$. From Pons et al. (2005)

The main advantage of this method is that the computation is not biased by the presence of high current peaks, and it is more reproducible from one cycle to another one. For instance, Benard et al. (2011) used it in a closed-loop airflow control application. Indeed, by such a method, only one AC period is sufficient to compute an accurate value of the consumed electrical power. This takes sense for closed-loop experiments where the loop time has to be small. In Benard et al. (2011), the instantaneous consumed power was continuously recorded with a capacitor $(47 \mathrm{nF})$ and the non-filtered Lissajous loops were integrated instead of approximating the charge diagram by straight lines such as in Kriegseis et al. (2011b) for instance. The estimation of the consumed power was computed in $1.8 \mathrm{~ms}$ by using a RJ45 communication between a digital oscilloscope and a National Instrument PXI card. This power estimation procedure differs by less than
$2 \%$ compared to time-averaged integration of the product of voltage and current over 20 AC cycles. A nominal uncertainty (standard deviation) of $4 \mathrm{~mW}$ was estimated. Recently, Ashpis et al. (2012) provide an extensive study that discusses the different methodologies for estimating the electrical power consumption.

Figure 7 presents the electrical power consumed by a 20-cm-long (in span wise) plasma actuator (Forte et al. 2007), as a function of the applied high voltage and the sine waveform frequency. As in Pons et al. (2005) and (Dong et al. (2008), these both curves demonstrate that in the case of a thick dielectric:

$P_{\text {elec }}=K \times f_{\text {AC }} \times\left(V-V_{0}\right)^{2}$

where $K$ is a constant coefficient depending on the actuator geometry, the dielectric material and the environmental conditions. Moreover, this expression is true if the discharge does not behave as a filamentary discharge. In the case of thin dielectrics, other authors such as Roth et al. (2005) and Enloe et al. (2004a) found that:

$P_{\text {elec }} \propto V^{n}$

with $n$ ranging from 2 up to 3.5. For instance, Fig. 8 shows the electrical power consumption versus applied voltage for two different frequencies with a thin dielectric (Enloe et al. 2004a). One can remark that the behavior of the curve is very different compared to Fig. 7 because the discharge becomes filamentary when the applied voltage is increased, resulting in a power consumption growth. Indeed, the power consumed by the plasma actuator is composed of the power dissipated by the surface discharge and the dielectric losses (Roth et al. 2005). As the power losses in the dielectric increase linearly with the voltage frequency and with the square of the voltage (dielectric losses $\propto f_{\mathrm{AC}} \times V^{2}$ ), the increase in the consumption with a power law higher than two comes from the discharge (see Fig. 9) because it
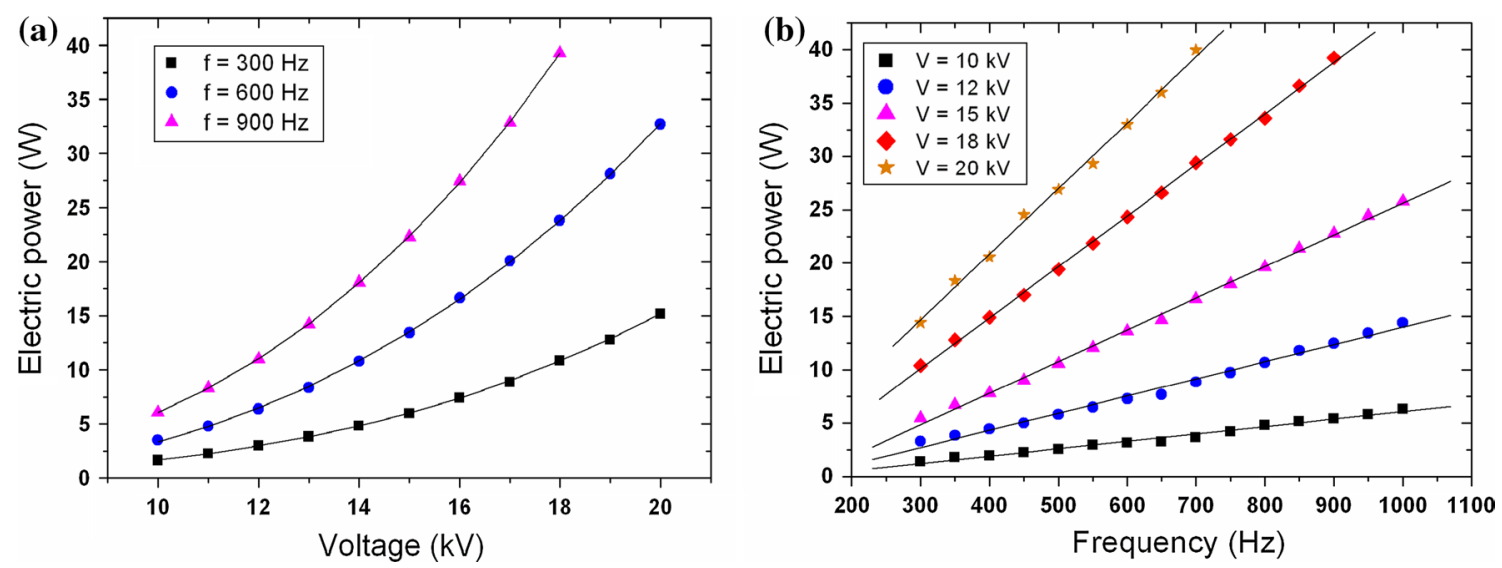

Fig. 7 Electrical power consumption versus applied voltage (a) and frequency (b) with a 2-mm-thick dielectric and 20-cm-long electrodes. From Forte et al. (2007) 


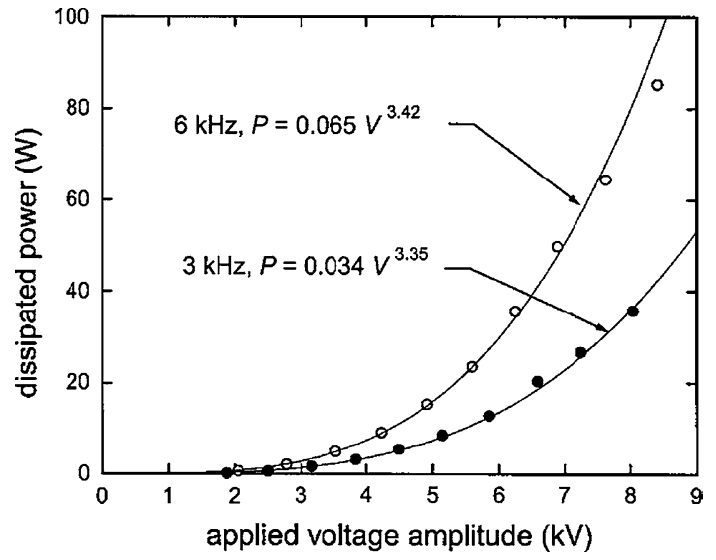

Fig. 8 Electrical power consumption versus applied voltage for two different frequencies with a thin dielectric. From Enloe et al. (2004a)

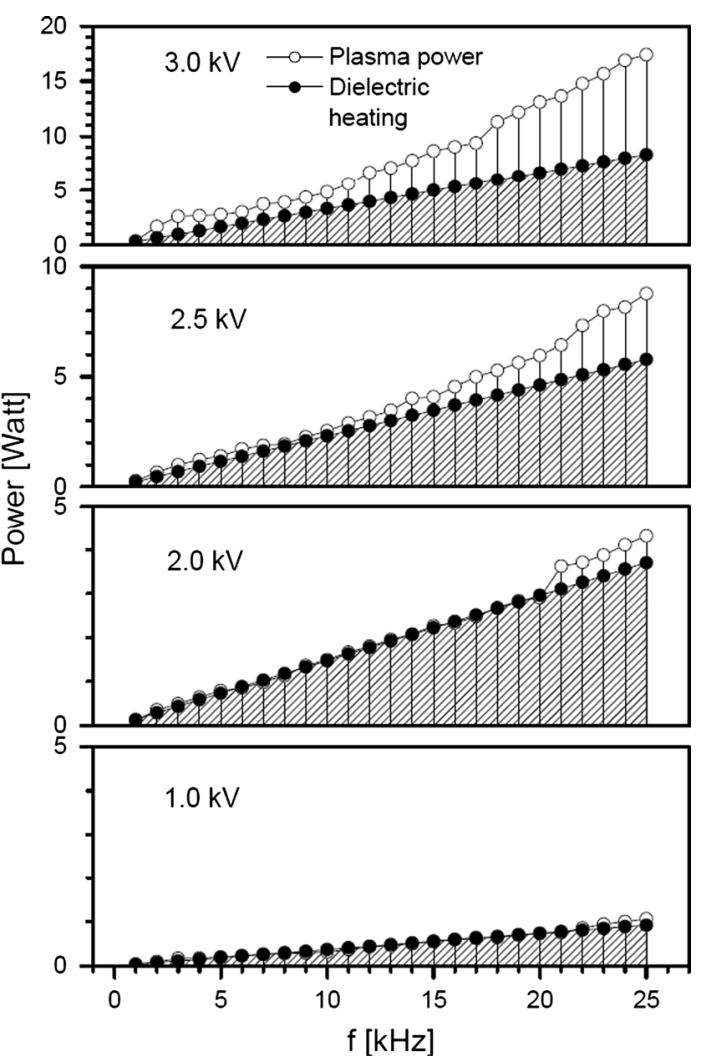

Fig. 9 Electrical power dissipation versus frequency (solid points correspond to the dielectric heating losses $P_{\mathrm{d}} \propto f_{\mathrm{AC}} \times V^{2}$ ). From Roth et al. (2005)

becomes filamentary when the voltage and the frequency increase above a threshold.

Usually, the electrical power consumption is expressed in $\mathrm{W}$ per electrode unit length $(\mathrm{W} / \mathrm{cm})$. The typical power consumed by plasma actuator ranges from 0.1 to a few W/ $\mathrm{cm}$, and in most cases of airflow control applications, its value is around $1 \mathrm{~W} / \mathrm{cm}$, whatever the dielectric thickness.

\subsection{Time-averaged discharge thrust and EHD force}

To characterize the mechanical effects of a plasma actuator, two methods are used. The first one consists in measuring the time-averaged thrust induced by the discharge. For such measurement, the measured thrust is not the EHD force but it corresponds to the EHD force produced by the discharge minus the total drag incurred by the plate supporting the actuator. This total drag comprises the viscous drag caused mainly by friction at the dielectric wall and form drag due to the pressure distribution around the plate. This type of measurement is simple and fast (a precision balance is sufficient), but it is a global one: It is not resolved in space, and usually, it is not resolved in time. The second method is based on the measurement of the electric wind velocity by systems used in fluid mechanics and aerodynamics, such as hot wire, LDV or PIV. In these latter methods, the flow measurement can be resolved in time and in space, and the force is derived from the velocity field by using Navier-Stokes equations with a few assumptions. In the present paper, both methods will be presented because they are complementary. In this section, the thrust produced by a single-surface DBD plasma actuator is characterized.

The time-averaged thrust produced by a surface discharge is usually measured with the help of laboratory balance. Its precision must be smaller than $0.1 \mathrm{mN}$ because the typical measured values range from a few $0.1 \mathrm{mN}$ to a few $\mathrm{mN}$ in the case of a 10-cm-long actuator. The DBD actuator is fixed vertically on the balance to measure the longitudinal force. Sometimes the balance is placed in a Faraday box to prevent electromagnetic interferences. A very large number of authors used this method to determine the force produced by surface discharge, such as (Enloe et al. 2004b; Debien et al. 2012a; Kriegseis et al. 2011b; Hoskinson and Hershkowitz 2010; Durscher and Roy 2011). Only three examples are presented in this section.

In 2004, Enloe's group published several papers in which the authors characterized the mechanical effect of surface DBD by force measurements (Enloe et al. 2004b; Van Dyken et al. 2004). The actuator was based on a 0.3-mmthick Kapton dielectric with different electrode shapes (plate and circular electrodes). First, they showed that the produced thrust was proportional to the dissipated power and that the geometrical parameters (such as the electrode shape) and electrical parameters (such as the voltage waveform) play a key role in the value of the produced force (this will be discussed further in the second part of this paper). By optimization, they reach a value of $0.9 \mathrm{~g}$ for an electrical consumption of $55 \mathrm{~W}$, i.e., an effectiveness equal to about $16 \mathrm{mg} / \mathrm{W}(0.15 \mathrm{mN} / \mathrm{W})$. For instance, Fig. 10a shows that the effectiveness in $\mathrm{g} / \mathrm{W}$ increases when the radius of the high-voltage electrode decreases. In similar 

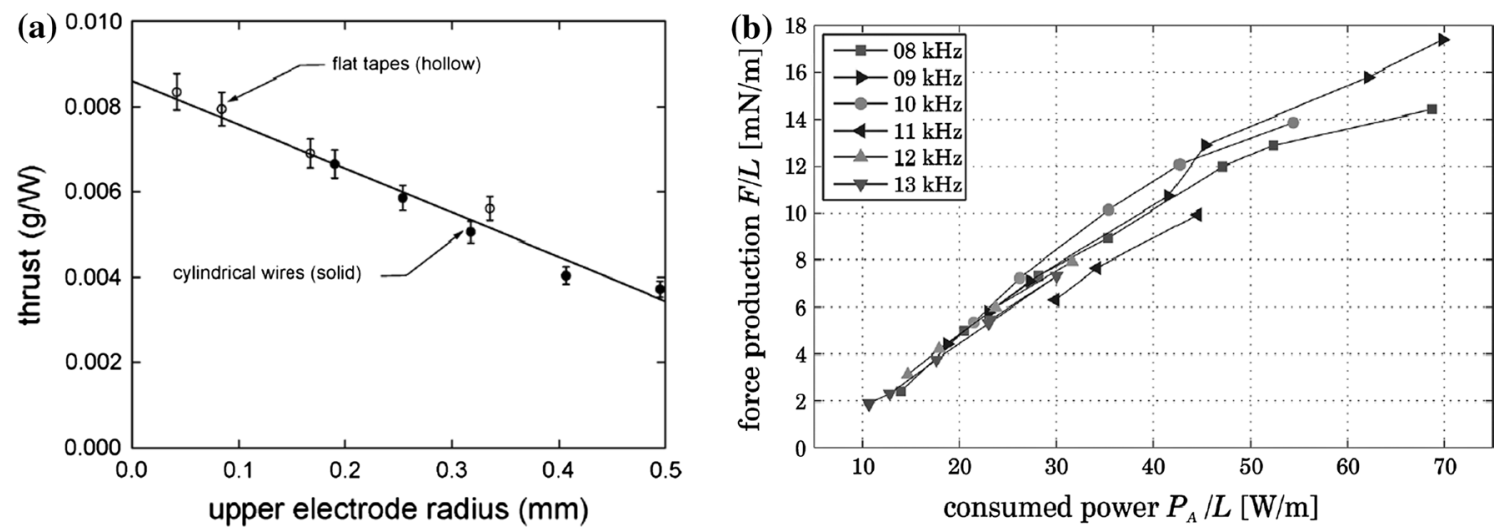

Fig. 10 Thrust produced by a thin dielectric-based DBD a by Enloe et al. (2004b) and b by Kriegseis et al. (2011b)
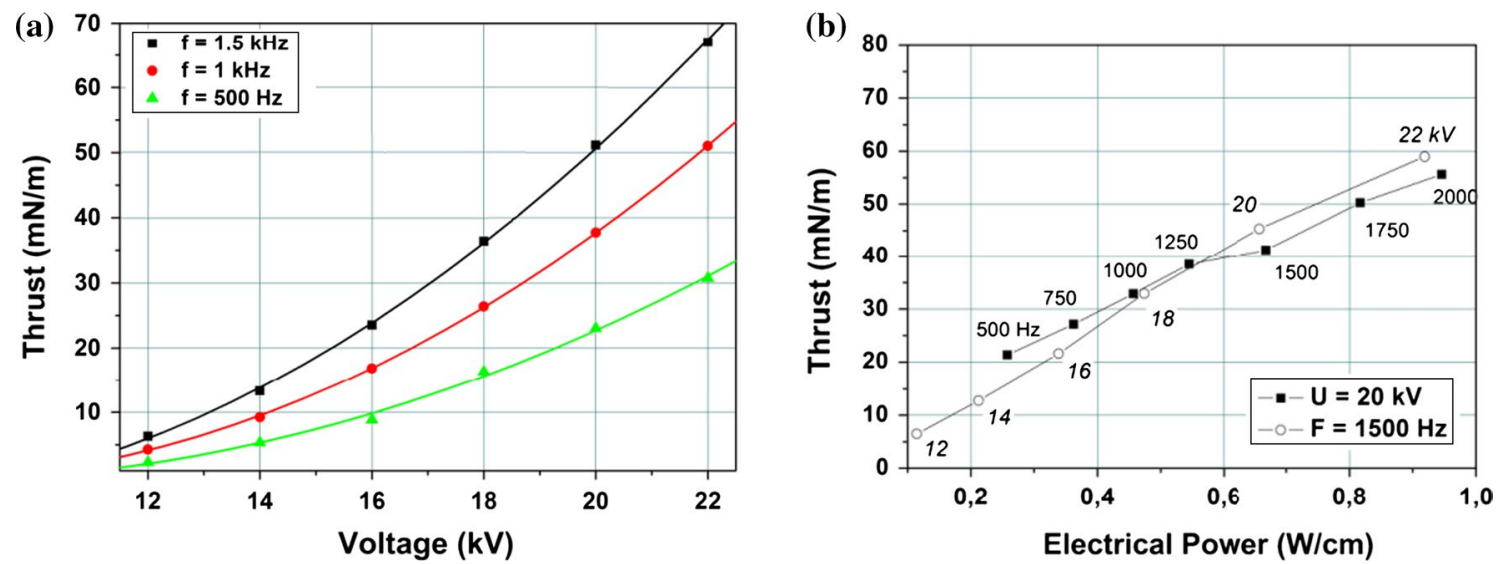

Fig. 11 Thrust versus applied voltage (a) and versus electrical power (b). Electrical power consumption is modified by an amplitude voltage variation at a fixed frequency set to $1,500 \mathrm{~Hz}$ and by a frequency driving signal variation with a voltage set to $20 \mathrm{kV}$. From Debien et al. (2012a)

conditions, Hoskinson and Hershkowitz (2010), Hoskinson et al. (2008b) obtained a value of about $0.2 \mathrm{mN} / \mathrm{W}$.

A more recent study (Kriegseis et al. 2011b) confirms that the thrust increases nearly linearly with the consumed power (Fig. 10b) with an effectiveness of about $0.25 \mathrm{mN} / \mathrm{W}$. However, one can remark a slight decrease in the curve when the power increases above about $50 \mathrm{~W} / \mathrm{m}$ $(0.5 \mathrm{~W} / \mathrm{cm})$ because the discharge becomes filamentary. This saturation effect had been previously observed by Thomas et al. (2009) with a 6.35-mm-thick dielectric when the electrical power consumption reached about $3 \mathrm{~W} / \mathrm{cm}$.

More recently, Debien et al. (2012a) measured the thrust $\mathrm{T}$ produced by a plate-to-plate single DBD using a 3 -mm-thick dielectric. Figure 11a presents the measured thrust versus applied high voltage for three $f_{\mathrm{AC}}$ frequency values. It highlights that measurements can be well fitted by the law $T \propto\left(V-V_{0}\right)^{2}$, such as the electrical power consumption. Figure $11 \mathrm{~b}$ shows force production versus electrical power consumption for a fixed frequency (set to $1,500 \mathrm{~Hz}$, voltage is increased from 12 to $22 \mathrm{kV}$ ) and a fixed amplitude voltage (set to $20 \mathrm{kV}$, frequency is adjusted from 500 to $2,000 \mathrm{~Hz}$ ). On the one hand, we can see that force is a quasi-linear function of electrical power consumption, as previously shown by Fig. 10. This is due to the fact that both electrical power and thrust increase with $\left(V-V_{0}\right)^{2}$. On the other hand, one can remark that the curve slope is higher when the voltage is increased at a constant frequency. This shows that it is more relevant to increase the voltage magnitude instead of the voltage frequency. Finally, in Fig. 11b, a thrust of about $60 \mathrm{mN} / \mathrm{m}$ is obtained when the consumed electric power is equal to $1 \mathrm{~W} / \mathrm{cm}$, resulting in an effectiveness of $0.6 \mathrm{mN} / \mathrm{W}$. This highlights that the force effectiveness increases with the thickness of the dielectric. Up to now, the strongest measured force is the thrust produced with a 6.35 -mm-thick quartz dielectric by Thomas et al. (2009). At $1 \mathrm{kHz}$, for voltages up to $40 \mathrm{kV}$, the authors measured force equal to 50 and $125 \mathrm{mN} / \mathrm{m}$ for power consumption of 1 and $4 \mathrm{~W} /$ $\mathrm{cm}$, respectively. This results in an effectiveness of 0.5 and $0.31 \mathrm{mN} / \mathrm{W}$, respectively. This shows that the force 
effectiveness (in $\mathrm{mN} / \mathrm{W}$ ) decreases when the electrical power consumption increases.

To conclude this section, note that one has to be careful with force measurements because the measured force is not the EHD force, but the total thrust (EHD force minus friction force). Because wall friction increases with the dielectric plate size, the size of the dielectric plate influences the measured value (Durscher and Roy 2011).

\subsection{Time-averaged electric wind velocity}

The EHD force created by a surface plasma actuator results in the production of a flow usually called electric wind or ionic wind. The main advantage of characterizing the electric wind velocity instead of the total thrust is that its measure can be resolved in space. The simplest method to conduct this type of experiments is to use a pressure probe as a Pitot tube connected to a manometer. However, a typical Pitot tube is made of stainless steel, and then, it can interact electrically with the discharge. Consequently, a glass home-made total pressure probe can be used, such as Léger et al. (2001) for the first time in the case of a surface DC corona actuator and Pons et al. (2004) in the case of a surface DBD actuator. This method is now widely used to characterize plasma actuators.

A typical experimental setup consists of a horizontal glass capillary (internal diameter ranging typically from 0.5 to $1 \mathrm{~mm}$ ) that can be displaced along both $x$ and $y$ axes (see Fig. 1 for $x$ and $y$ axes) with the help of a step-by-step motor with a spatial accuracy smaller than $0.1 \mathrm{~mm}$. For instance, in Debien et al. (2012a), a 0.6-mm-diameter glass tube is used to measure the pressure resulting from the produced electric wind. Pressure is measured by an analogical manometer, and the time-averaged horizontal component of the electric wind velocity is determined with Bernoulli equation. In such conditions, standard deviation of the electric wind measurements is usually smaller than $0.05 \mathrm{~m} / \mathrm{s}$ if sampling is high enough and convergence is ensured.

Figure 12a shows a typical example of horizontal velocity profile along $y$ axis for $x$ several locations. One can see that the surface DBD produces a jet that seems parallel to the wall (note that this pressure measurement gives only the horizontal component of the velocity). At $x=15 \mathrm{~mm}$ (15 $\mathrm{mm}$ downstream the edge of the $\mathrm{HV}$ active electrode), the maximum velocity is here equal to $5 \mathrm{~m} / \mathrm{s}$, and it is measured at about $0.8 \mathrm{~mm}$ above the wall. The jet thickness is equal to a few mm. Further downstream, the maximum velocity decreases as the jet thickness increases due to diffusion and viscous effect. Figure $12 \mathrm{~b}$ presents a vertical profile (along $x$ axis) in the case of an optimized actuator (4-mm-thick dielectric, $V=30 \mathrm{kV}, f_{\mathrm{AC}}=1.5 \mathrm{kHz}$ ) at $0.9 \mathrm{~mm}$ above the wall. It highlights that the horizontal component of the electric wind velocity increases from $x=0$ to about $10-15 \mathrm{~mm}$. The maximum velocity is equal to $7 \mathrm{~m} / \mathrm{s}$. To our knowledge, it is the highest value that has been measured with a single DBD up to now. Then, the velocity reaches a plateau (at $7 \mathrm{~m} / \mathrm{s}$ ), and it decreases slowly downstream the discharge region because there is no EHD force.

Indeed, it has been demonstrated previously that the discharge extension along $x$ increases with the applied voltage (see Fig. 5) and that the location at which the maximum electric wind velocity occurs corresponds nearly to the discharge extension limit (Kriegseis et al. 2011b; Forte et al. 2007). To confirm this assumption, the location $X_{U \max }$ at which the maximum electric wind velocity occurs has been determined by Jolibois and Moreau (2009) by pressure probe measurements. On the one hand, Fig. 13 shows that this location increases linearly with the applied voltage amplitude, whatever the dielectric thickness in a range from 0.5 to $5 \mathrm{~mm}$. For operating high voltage of $25 \mathrm{kV}$, the electric wind velocity accelerates up to $x \approx 14 \mathrm{~mm}$. On the other hand, Fig. 5 has shown that the plasma extension was limited to a few $\mathrm{mm}$ in the case of a thin dielectric-based DBD actuator. Then, the value of $X_{U \max }$ is smaller with a very thin dielectric (in the range 50-300 $\mu \mathrm{m}$ ). To summarize, the use of a thick dielectric results in a more effective and more robust actuator because the maximum velocity, the jet thickness and the flow-plasma interaction surface are higher.
Fig. 12 Electric wind velocity profile, along $y$ at different $x$ values (3-mm-thick dielectric, $\left.V=24 \mathrm{kV}, f_{\mathrm{AC}}=1 \mathrm{kHz}\right)(\mathbf{a})$ and along $x$ at $y=0.9 \mathrm{~mm}$ (4-mm-thick dielectric, $\left.V=30 \mathrm{kV}, f_{\mathrm{AC}}=1.5 \mathrm{kHz}\right)(\mathbf{b})$
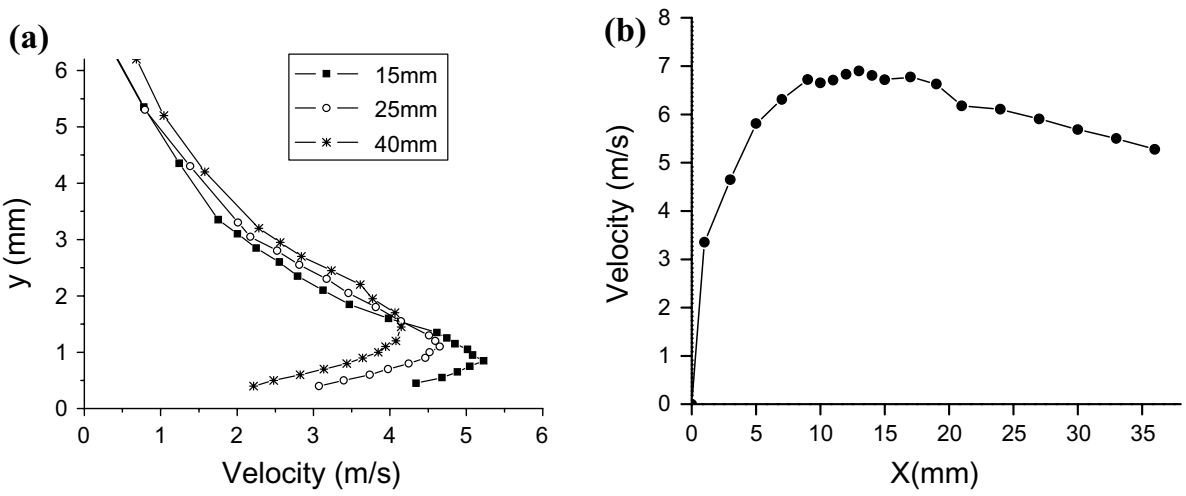


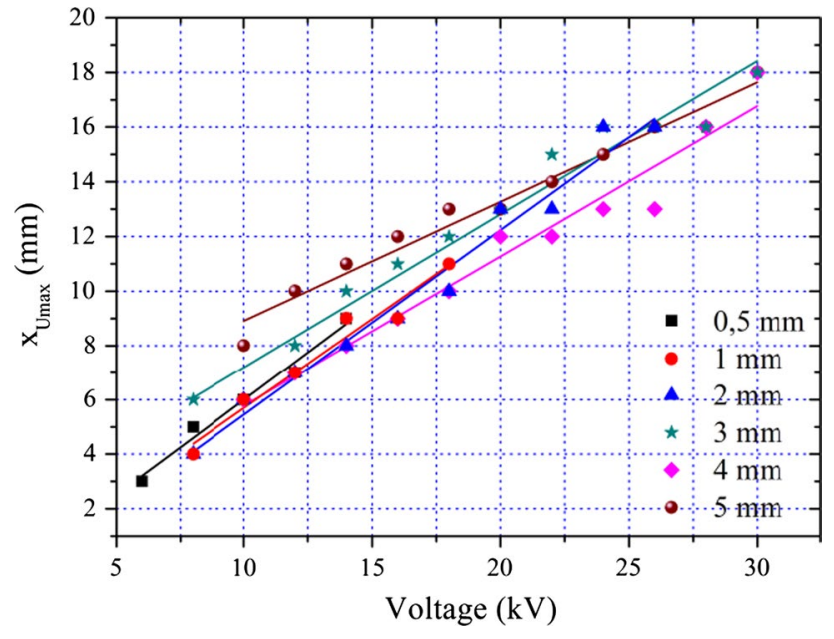

Fig. 13 Location along $X$ where the maximum electric wind velocity is measured versus applied high voltage, for several dielectric thickness values. From Jolibois and Moreau (2009)

Figure 14 shows the evolution of the maximum velocity $U_{\max }$ above the actuator with the voltage amplitude $V$ and the frequency of the sine waveform $f_{\mathrm{AC}}$. If one focuses on the frequency curve (Fig. 14a), we can see that the velocity increases with frequency. This is due to the fact that the charge transferred per period $T_{\mathrm{AC}}$ remaining nearly constant, the mean transferred charge increases with the frequency, inducing more collisions between ions and gas. Indeed, the EHD force can be simply expressed by:

$\vec{F}_{\text {EHD }}=\rho \times \vec{E}$

where $\rho$ is the space charge in $\mathrm{C} / \mathrm{m}^{3}$ and $E$ the electric field in $\mathrm{V} / \mathrm{m}$. An increase of $f_{\mathrm{AC}}$ results in an increase of $\rho$. However, for frequency higher than about $1.2 \mathrm{kHz}$ in this case, the maximum velocity reaches a plateau because electrical charges deposited and accumulated at the dielectric wall do not have time enough between two successive discharges to fully relax. Then, the surface discharge becomes filamentary and the resulting energetic filaments do not contribute to electric wind production. For the voltage curve (Fig. 14b), the evolution is not asymptotic in the range 8-26 kV because one can see that the maximum velocity does not reach a plateau. This evolution means that faster velocities should be reached with higher voltages. Moreover, one can note that the velocity fluctuations increase slightly with the applied voltage (the rms values are plotted with error bars in Fig. 14b).

These both curves demonstrated that it is more effective to increase the high voltage instead of the frequency. Indeed, the voltage enhances both $\rho$ and $E$. Because the value of $\rho$ is limited by the glow-to-filament transition, it is better to increase $\mathrm{E}$.

Finally, Fig. 15a presents the maximum electric wind velocity versus electrical power consumption, for several dielectric thickness values. It highlights three very interesting results: (1) For a given electric power consumption, the maximum velocity does not depend on the dielectric thickness in this thickness range, (2) the maximum velocity depends only on the electrical power consumed by the actuator, (3) up to $2 \mathrm{~W} / \mathrm{cm}$, the maximum velocity increases with the power, but there is a saturation effect after $2 \mathrm{~W} / \mathrm{cm}$. Others results can be found in Jolibois and Moreau (2009).

From velocity profiles as in Fig. 12a, the mass flow rate $Q$ produced by the discharge can be computed by:

$Q=\rho_{G} \int_{y=0}^{\infty} U(y) \mathrm{d} y$

where $\rho_{G}$ is the air density and $U(y)$ the measured velocity above the dielectric plate, from the wall to the maximum value
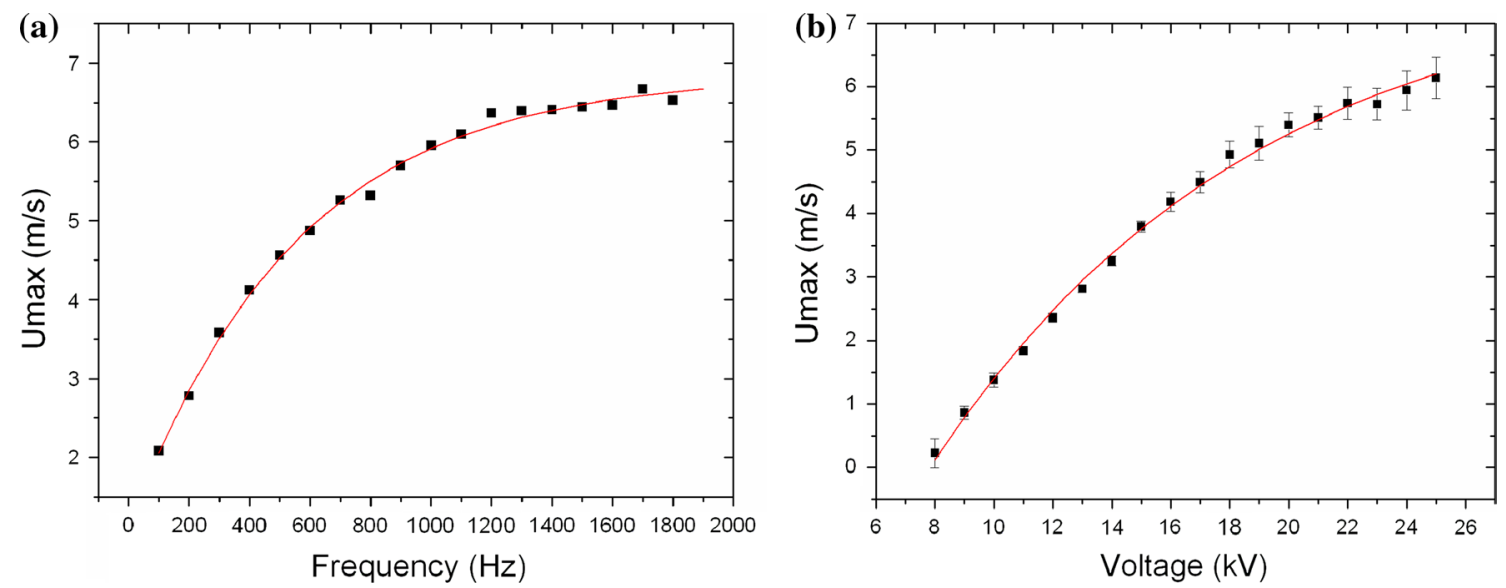

Fig. 14 Maximum electric wind velocity $U_{\max }$ versus frequency (2-mm-thick dielectric, $\left.V=20 \mathrm{kV}\right)($ a) and maximum electric wind velocity $U_{\max }$ versus voltage $\left(f_{\mathrm{AC}}=1 \mathrm{kHz}\right)(\mathbf{b})$. From Forte et al. (2007) 

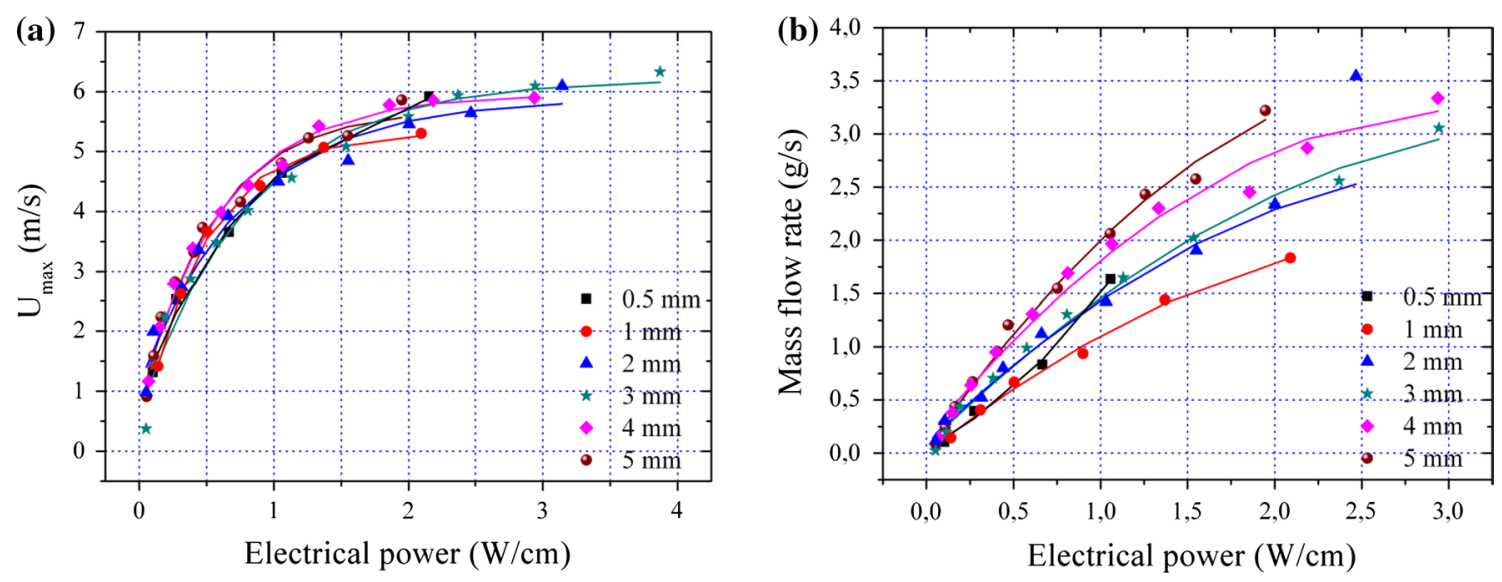

Fig. 15 Maximum electric wind velocity (a) and mass flow rate (b) versus electrical power consumption for several dielectric thickness values. From Jolibois and Moreau (2009)

of $y$ for which a velocity can be detected. Figure $15 b$ that presents $Q$ versus $P_{\text {elec }}$ shows that the mass flow rate increases up to a few $\mathrm{g} / \mathrm{s}$ with the electrical power. Moreover, it highlights that the mass flow rate is higher with a thicker dielectric. This is due to the fact that the thickness of the electric wind jet increases with the thickness of the dielectric plate.

\subsection{Time-averaged electric wind topology}

The main advantage of the pressure probe measurement described in the previous section is that it is weakly intrusive (at least when the pressure probe is not located in the discharge region), simple and low cost. However, it does not allows us to measure both components of the electric wind velocity. To characterize the electric wind topology, 2D velocity fields can be obtained by particle image velocimetry (PIV), such as in Léger et al. (2001, 2002) for the first time. Several authors, as Kriegseis et al. (2011b) and Kotsonis and Ghaemi (2011) among others, use now this type of system. A sketch of such a system is given in Fig. 16. Small seeding particles are introduced in a large box containing the actuator and quiescent air. When the discharge is switched on, the produced flow results in the displacement of the particles. This displacement can be quantitatively characterized by computing cross-correlations between two successive images of the field of view. Algorithms are based on crosscorrelation of sub-regions of the global images to finally result in a spatially resolved distribution of the velocity field.

With such a system, one has to be careful because the measure can be strongly biased. It has been the case in several published papers. The first problem concerns the particles that are used to seed the flow. If they are electrically charged above a given threshold, they can move under their own EHD force (Coulomb force applied on a charged particle), and then, they do not follow the fluid motion. In

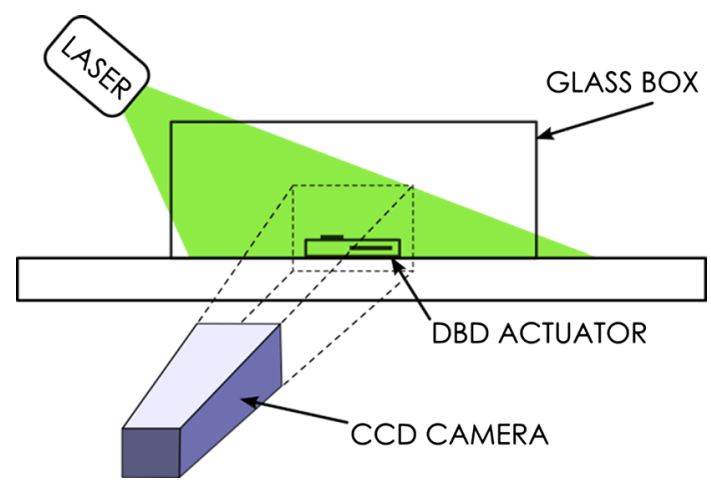

Fig. 16 Sketch of the PIV system for the characterization of the electric wind topology

this case, there is a slipping effect between the particles and the fluid. Then, the measured velocity does not correspond to the electric wind velocity. Particles produced by atomization of liquid oil (cosmetic and olive oil) solve this issue. But, the particle diameter must be equal to about $0.3-0.4 \mu \mathrm{m}$ (at least smaller than $1 \mu \mathrm{m}$ ) to limit the charging effect, and the particle rate in air must be correctly adjusted. The second problem is the PIV system itself. The choice of the interrogation size for the cross-correlation and the delay time between two successive images are two parameters (among others) that can significantly affect the accurate estimation of the electric wind in the vicinity of the boundary layer. In fact, PIV measurement must be compared to a pressure probe measurement in order to be sure that there is no difference between both measurements. Third, the box must be large enough to avoid any flow recirculation. As an example of biased measurements on previous published papers, one can indicate the case of flow fields with a maximum electric wind velocity far downstream the discharge extension is not possible because 
Fig. 17 Time-averaged electric wind produced by a 3-mmthick dielectric $(V=22 \mathrm{kV}$, $f_{\mathrm{AC}}=1.5 \mathrm{kHz}$ ): velocity vectors and norm of the horizontal velocity component in graylevel background (a), streamlines and vertical velocity component in gray-level background (b) and colored flow field (c). d Time-averaged electric wind produced by a $110-\mu \mathrm{m}$-thick dielectric actuator. Scale in millimeter. a-c From Debien et al. (2012a), d from Kotsonis and Ghaemi (2011) (a)

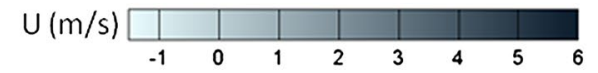

(b)
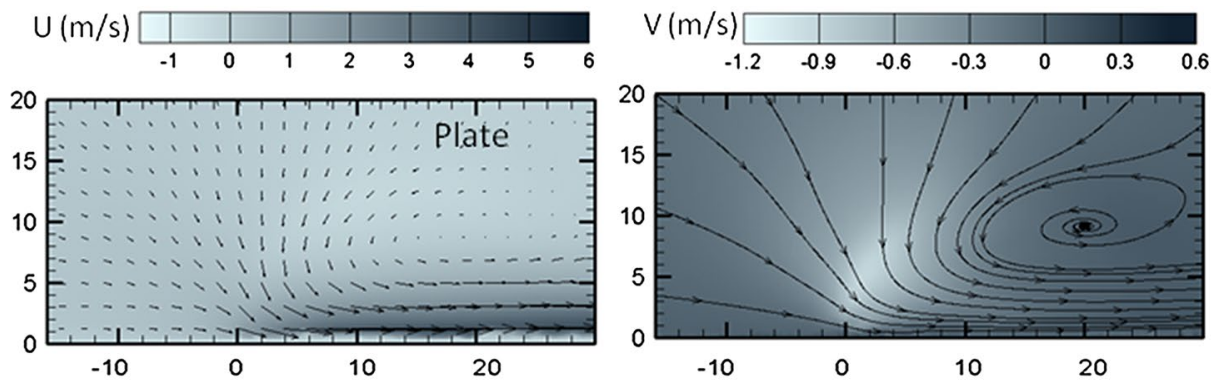

(c)

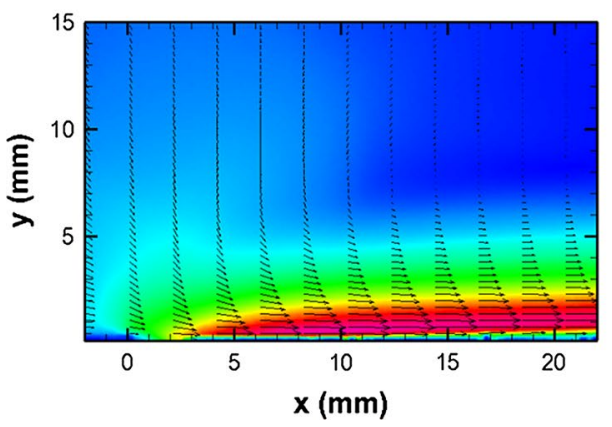

(d)

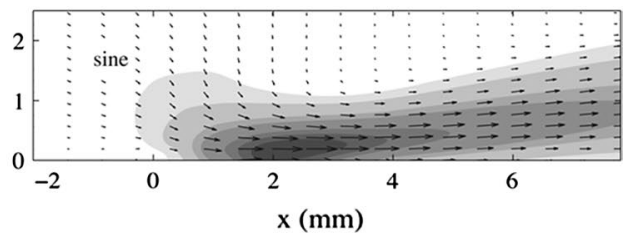

there is no EHD force. The maximum velocity is always located at the discharge extension $(5-15 \mathrm{~mm})$ and it cannot be measured further downstream.

For instance, time-averaged velocity fields obtained from PIV experiments are shown in Fig. 17. As explained previously, the aim of this velocity field is mainly to observe the topology of the produced electric wind. Figure 17a, b shows the velocity vectors and norm of the horizontal velocity component in gray-level background, and the streamlines and vertical velocity component in gray-level background, respectively. First, one can observe that a wall jet is created from the active electrode edge to downstream. The horizontal component of the electric wind velocity measured here with the PIV system is similar to the one previously determined with a pressure probe, but the PIV acquisitions bring more details on the fluid suction developing above the discharge $(-10 \leq x \leq 10 \mathrm{~mm})$. In this case, the suction induces a vertical velocity which achieved $-0.9 \mathrm{~m} / \mathrm{s}$.

Figure $17 \mathrm{c}$ presents another example of electric wind characterization by PIV experiments, with a better spatial resolution $(100 \mu \mathrm{m})$. It confirms that the maximum electric wind velocity is measured at about $0.5 \mathrm{~mm}$ above the wall. It allows us to observe more accurately the velocity profiles at different $x$ values. In Fig. 17d, one can see the flow field in the case of a thin dielectric (110 $\mu \mathrm{m}$ of Kapton). It highlights that the use of thin dielectric results in two effects: the discharge propagation being limited to a few $\mathrm{mm}$, the region of velocity production is smaller compared to Fig. 17c, and consequently, the suction effect is reduced.

\subsection{Time-resolved electric wind velocity}

Another manner to characterize more accurately the produced flow is to investigate its time-resolved behavior. Time-resolved measurements can be achieved with the help of a high-speed PIV system, LDV (laser Doppler velocimetry) or hot wire. The last method cannot be used close to the discharge because the electrical disturbances. As for PIV, LDV needs particles to seed the flow. Then, the same precautions must be taken.

The first measurements of the electric wind velocity versus time have been performed in 2005 by Forte et al. (2005) (Fig. 18a, b). In these experiments, a voltage of $18 \mathrm{kV}$ was applied and the dielectric thickness was equal to $1.5 \mathrm{~mm}$. The frequency $f_{\text {AC }}$ was equal to $700 \mathrm{~Hz}$. The actuator was placed in a $1 \mathrm{~m} / \mathrm{s}$ velocity-free flow. From Fig. 18a, b, one can see that a fast acceleration is clearly observed when the discharge is switched on $(t=0.5 \mathrm{~s}$ after the beginning of the LDV acquisition) with a rise time of about $10 \mathrm{~ms}$. Indeed, at the discharge initiation (at $t=0.5 \mathrm{~s}$ ), the averaged value of the velocity rises from $1.5 \mathrm{~m} / \mathrm{s}$ to about $5 \mathrm{~m} / \mathrm{s}$ above the actuator, in the boundary layer. The plot also shows clearly that the flow is quite unstable, and strong oscillations at the same frequency than the sine high voltage can be observed. If this part of the plot is zoomed (Fig. 18b), 35 pulses appear in a duration window of $50 \mathrm{~ms}$, meaning that the fluid is pulsed at a frequency of $700 \mathrm{~Hz}$. This measurement had proved for the first time that the DBD does not act similarly during the negative and the 

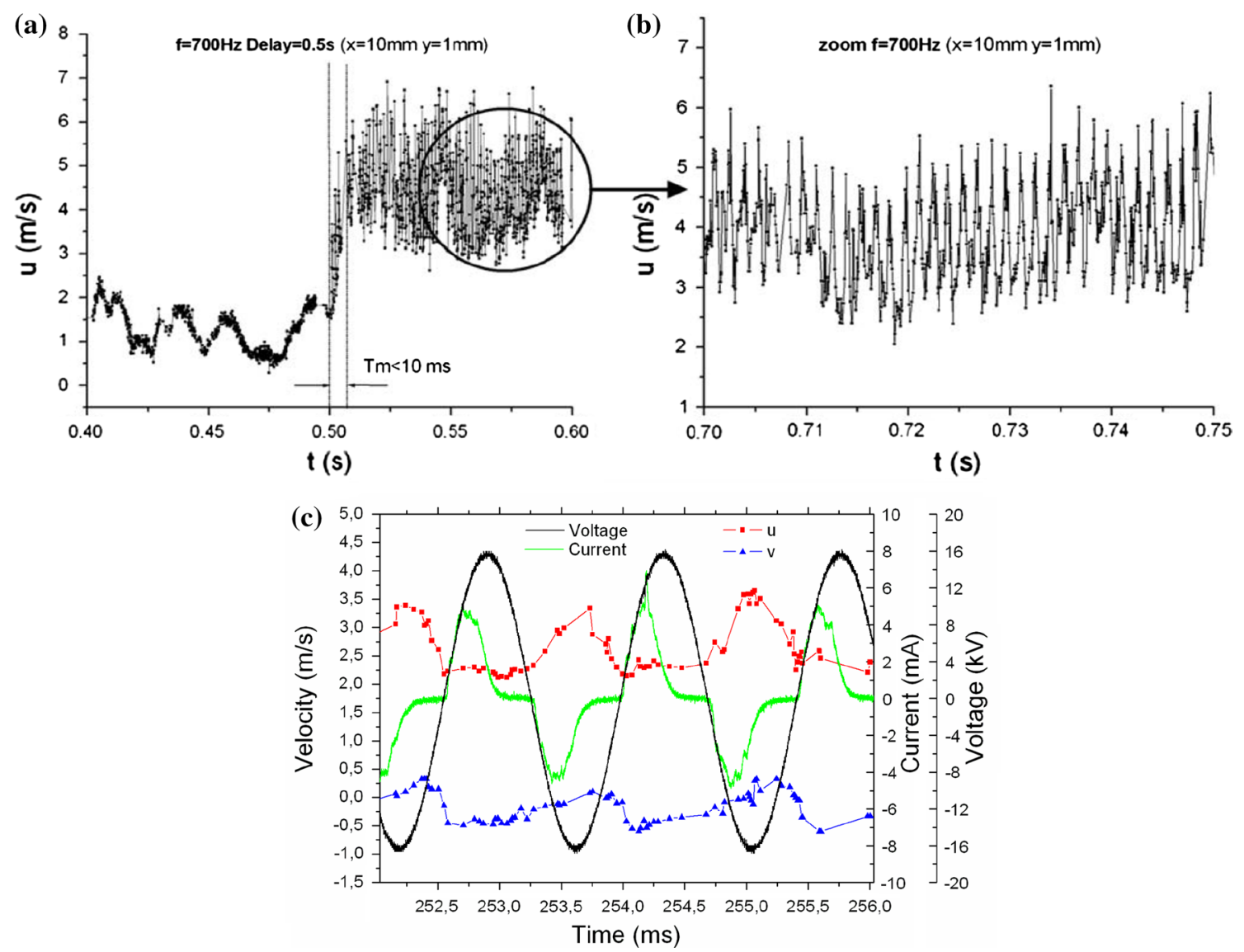

Fig. 18 Electric wind velocity versus time: $f_{\mathrm{AC}}=700 \mathrm{~Hz}, x=10 \mathrm{~mm}, y=1 \mathrm{~mm}(\mathbf{a}, \mathbf{b})$ and $f_{\mathrm{AC}}=700 \mathrm{~Hz}, x=2 \mathrm{~mm}, y=1 \mathrm{~mm}(\mathbf{c})$. From Forte et al. $(2006,2007)$

positive half-cycles. However, the power supply and the LDV system were not synchronized in time; we were then unable to know what going cycle was the most effective in velocity production.

Consequently, in 2006, similar experiments have been conducted with a system including synchronization between the sine high voltage and the velocity measurements (Forte et al. 2006, 2007). One plot is presented in Fig. 18c. The black and the green curves represent the applied sine highvoltage and the filtered discharge current, respectively. The red and the blue curves plot the horizontal and vertical components of the velocity, respectively. One more time, one can see that the surface DBD generates a pulsed flow at the same frequency as the high voltage. Both half-cycles generate horizontal velocity ( $U$ component), but the negative halfcycle induces more velocity $(3.6 \mathrm{~m} / \mathrm{s})$ than the positive one $(2.4 \mathrm{~m} / \mathrm{s})$ in the $x$ direction. These experiments had proved that the glow discharge occurring during the negative-going cycle was more efficient in velocity production than the streamer discharge that acts during the positive cycle.

More recently, further studies have been conducted. For instance, Benard and Moreau $(2009,2012)$ studied the non-stationary phenomena of the electric wind produced by several high-voltage waveforms. In these both papers, the electric wind was measured by a laser Doppler velocimetry system allowing measurements of two velocity components, with a high bandwidth that guarantees a timeresolved description of the induced flow. In order to reduce the background noise, acquisitions were performed over 600,000 data points (at a mean acquisition rate of $20 \mathrm{kHz}$ ) and an averaging process per class was applied. This average was based on 4,000 data points per class of $13.3 \mu \mathrm{s}$ in duration. Figure 19a shows the velocity versus time at $y=1 \mathrm{~mm}$ and $x=5 \mathrm{~mm}$, in the case of a sine high voltage. Several remarks can be done. First, it can be observed that the periods of $U$ velocity production correspond to the negative-going cycle of the applied voltage. In contrast, the period of streamer discharge (positive-going cycle) does not contribute to the production of velocity $U$. It results that the horizontal velocity component is the mirror of the input electrical waveform, revealing a quasi-linear electromechanical conversion phenomenon, with a phase-shift, this phase shifting depending on the measurement location. During the negative-going cycle, the velocity acceleration 


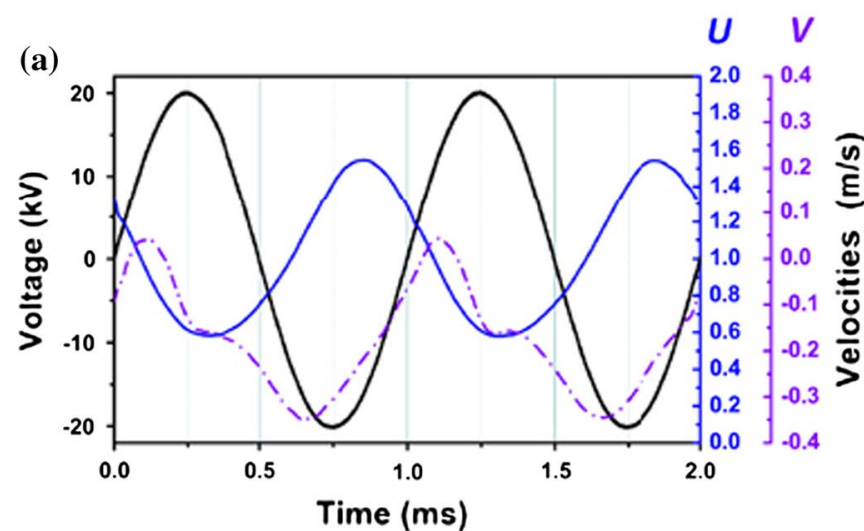

Fig. 19 Electric wind velocity versus time from LDV measurements $\left(f_{\mathrm{AC}}=1 \mathrm{kHz}, x=5 \mathrm{~mm}, y=1 \mathrm{~mm}\right)(\mathbf{a})$ and electric wind velocity versus time from PIV measurements $\left(f_{\mathrm{AC}}=625 \mathrm{~Hz}, x=2 \mathrm{~mm}\right.$,

is equal to about $3,000 \mathrm{~m} / \mathrm{s}^{2}$ at the selected measurement location. Secondly, the behavior of the vertical velocity component $\mathrm{V}$, that is mainly negative, indicates that the mean flow is deviated toward the dielectric wall, more especially during the negative-going cycle of the applied high voltage. The attraction toward the wall region should result from the pressure gradient in the vertical direction promoted by the flow acceleration and deceleration in the horizontal direction. As a result, when the glow discharge occurs, the horizontal velocity $U$ increases, promoting a suction effect above the actuator.

The behavior of the electric wind velocity as a function of time can be also studied with the help of a PIV system. In such a case, the camera and the laser used by the system must be able to work at high frequency (at least a few $\mathrm{kHz}$ ). Another manner is to reconstruct phase-averaged velocity fields from non-time-resolved measurements. To our knowledge, three groups conducted this type of analysis (Debien et al. 2012a; Kotsonis and Ghaemi 2011; Joussot 2011).

Kotsonis and Ghaemi (2011) studied the time history of the flow produced by a $110-\mu \mathrm{m}$-thick Kapton actuator. From phase-averaged velocity fields, they extracted the horizontal and vertical components of the electric wind velocity, at $x=2 \mathrm{~mm}$ and $y=0.2 \mathrm{~mm}$ (Fig. 19b). One can see that the behavior of both velocity components is very similar to the ones measured by Benard and Moreau with a LDV system, in the case of 3-mm-thick actuator (Benard and Moreau 2012). The difference in the measured electric wind velocity is due to the measurement location (in the plasma region for Kotsonis and Ghaemi 2011 and outside this region in Benard and Moreau 2012), but Fig. 19 demonstrates that the time behavior of the electric wind does not depend on the dielectric thickness.
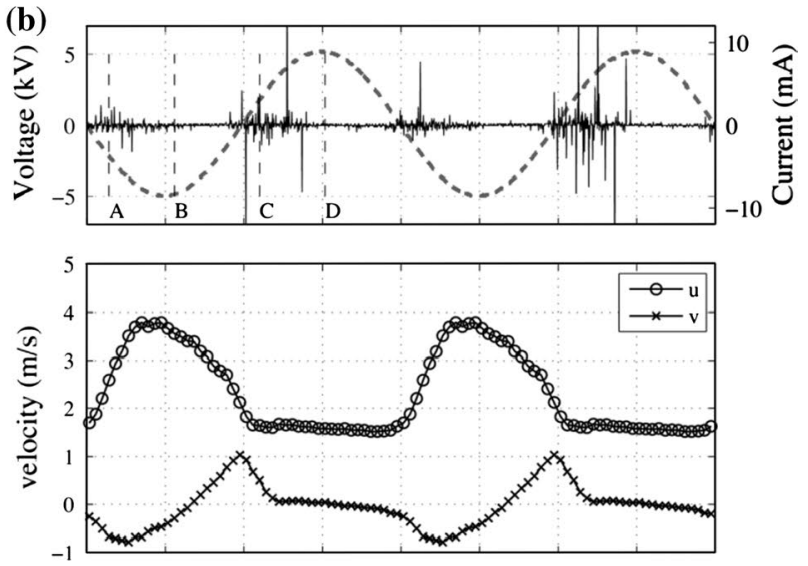

$y=0.2 \mathrm{~mm}$ ) (b). a From Benard and Moreau (2012), b from Kotsonis and Ghaemi (2011)

In 2012, Debien et al. (2012a) confirmed this behavior. With a high spatial resolution PIV system, the authors used a phase-averaged method to reconstruct the time behavior of the flow with a time resolution of $50 \mu \mathrm{s}$. First, time-averaged velocity fields were computed with 300 instantaneous velocity fields acquired at an interval of $200.005 \mathrm{~ms}$, chosen to correspond to a non-harmonic frequency of the $\mathrm{HV}$ actuation frequency. In a second time, twenty phase-averaged velocity fields per AC voltage cycle (time resolution of $50 \mu \mathrm{s}$ ) were computed from 260 instantaneous velocity fields acquired via a high-resolution PIV system (flow field of $26 \times 26 \mathrm{~mm}^{2}$ with a spatial resolution of $100 \mu \mathrm{m})$. To illustrate the unsteady behavior of the produced flow, Fig. 20 presents the electric wind velocity fields at two instants: $t^{*}=0.3$ that corresponds to the end of the streamer discharge occurring during the positivegoing cycle (see Fig. 2) and $t^{*}=0.55$, corresponding to the beginning of the glow discharge that ignites during the negative-going cycle. One can see that at $t^{*}=0.3$, the produced velocity is low, and it is mainly parallel to the wall in the discharge region. At $t^{*}=0.55$, the electric wind wall jet is thicker, and the vectors are deviated toward the wall, showing a more intense suction effect above the discharge. Time evolution of the horizontal velocity at four discrete points located at $1 \mathrm{~mm}$ above the wall is shown in Fig. 21 . One more time, one can observe that the plasma discharge induces a horizontal velocity at the same frequency that the high-voltage frequency. Horizontal velocity fluctuations at $x=5$ and $10 \mathrm{~mm}$ are characterized by a velocity increase at the ignition of the negative discharge $\left(t^{*} \approx 0.4\right.$, see Fig. 3$)$ and a decrease when it switches off $\left(t^{*} \approx 0.8\right)$. One can notice that the mean velocity increases from $x=5$ to $15 \mathrm{~mm}$ ( 3.1 to $4.2 \mathrm{~m} / \mathrm{s}$, respectively) because the flow accelerates up to the discharge extension, as explained 
Fig. 20 Velocity vector fields $(0-6 \mathrm{~m} / \mathrm{s})$ of electric wind at $t^{*}=0.3(\mathbf{a})$ and $t^{*}=0.55(\mathbf{b})$. $V=24 \mathrm{kV}, f_{\mathrm{AC}}=1 \mathrm{kHz}$. From Debien et al. (2012a)

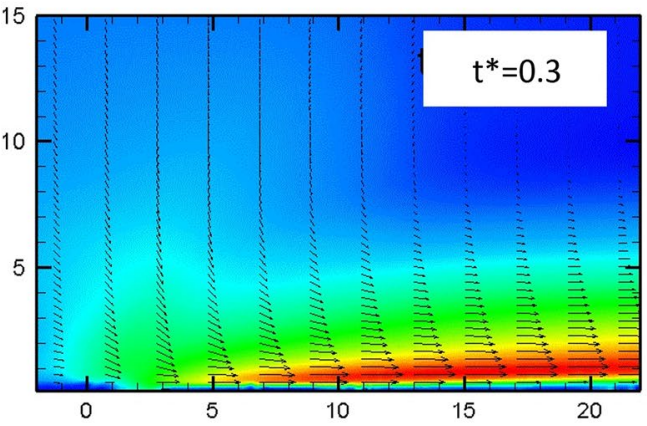

(a)
$\mathrm{U}(\mathrm{m} / \mathrm{s})$

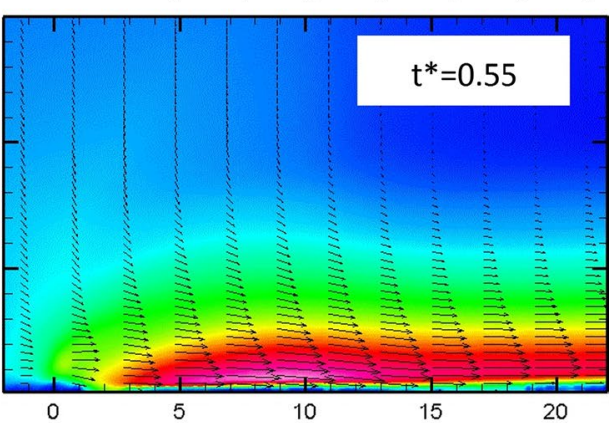

(b)

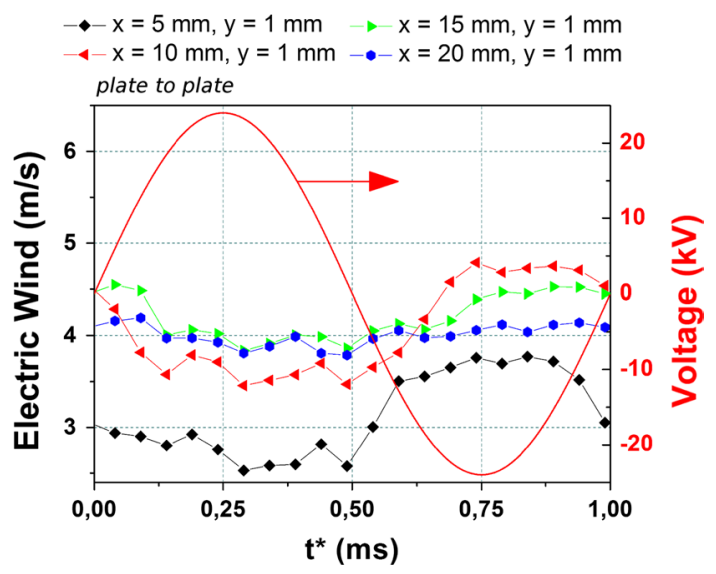

Fig. 21 Electric wind velocity versus time at different $(x, y)$ locations. From Debien et al. (2012a)

previously. These last observations are in agreement with results given in Figs. 18 and 19.

\subsection{Response time of the actuator in quiescent air}

In this section, one focuses on the time necessary to the discharge to transfer momentum to the fluid at the initiation. To fully understand how the flow reacts to the momentum transfer initiated by the plasma, time-resolved acquisitions are needed. In Benard and Moreau (2009, 2010), LDV measurements and phase reconstruction have been performed with a high temporal resolution $(20 \mu \mathrm{s})$ in order to characterize the instantaneous electric wind behavior when the discharge is switched on. One thousand modulated cycles were considered for each investigated case. For instance, Fig. 22 shows the local electric wind velocity versus time at $x=5 \mathrm{~mm}$ and $y=0.3 \mathrm{~mm}$. Results indicate that the momentum transfer is strongly non-stationary. When the discharge is switched on, during the first cycle, the velocity production is mainly related to the negative-going cycle of the voltage. After the first

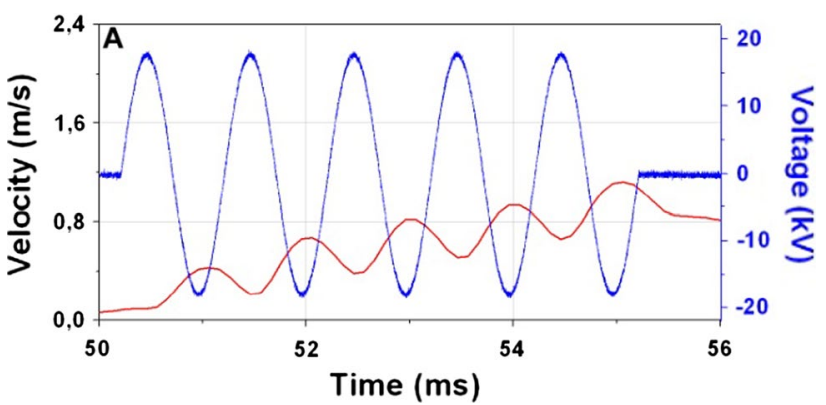

Fig. 22 Phase reconstruction of the electric wind production for $V=18 \mathrm{kV}$ at $f_{\mathrm{AC}}=1 \mathrm{kHz}, f_{\mathrm{BM}}=20 \mathrm{~Hz}$ and $\mathrm{DC}=10 \%$ (measurement location at $x=5 \mathrm{~mm}$ and $y=0.3 \mathrm{~mm}$ ). Voltage in blue and velocity in red. From Benard and Moreau (2009, 2010)

cycle, the positive-going cycle leads to a fluid deceleration. The difference results in an airflow increase with oscillations at the frequency of the electric signal $f_{\mathrm{AC}}$. The present results reveal that each $\mathrm{AC}$ cycle contributes to the velocity production by a cumulative effect. When the discharge is turned off, the surrounding airflow naturally relaxes.

The drawback of the previous measurement is that it gives the actuator response time at a local position, very close to the active electrode $(x=5 \mathrm{~mm})$ in Fig. 22. Another manner to characterize the response time is to observe the whole flow when the discharge is switched on. For instance, Moreau et al. (2008a) conducted Schlieren visualizations because they do not require to seed the flow with particles. In fact, this technique enables the visualization of flows in which density gradients are present, because the gray level of the image is proportional to the gas density. To visualize the electric wind effect, two vertical jets of Helium were injected above the plate wall (see Fig. 23a). The first one was located upstream the HV electrode when the second one was located at about $6 \mathrm{~cm}$ downstream. On the one hand, in the absence of airflow, both helium jets have a vertical trajectory because the density of helium is smaller than the one of the air. On the other hand, both 


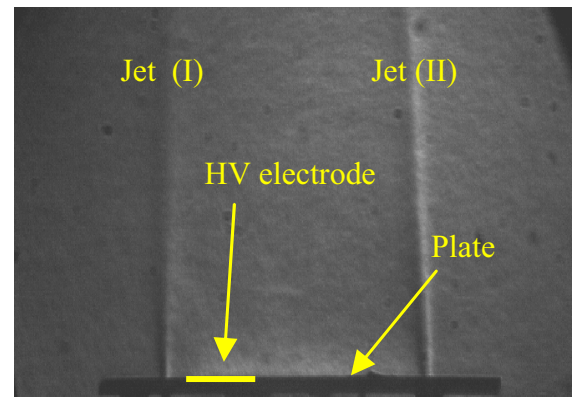

(a)

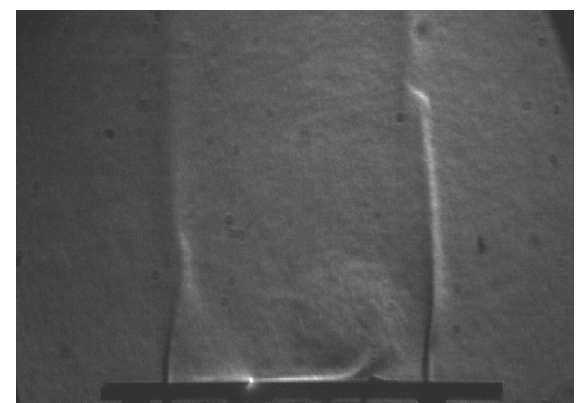

(d)

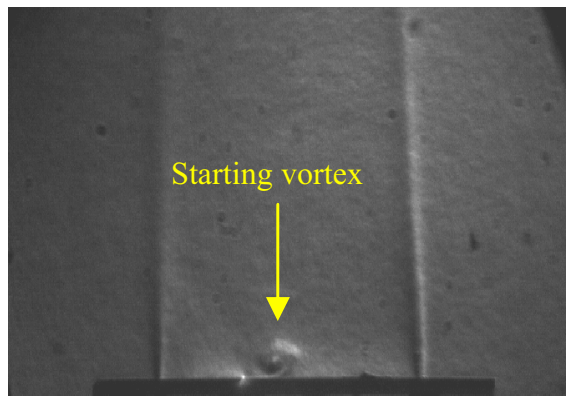

(b)

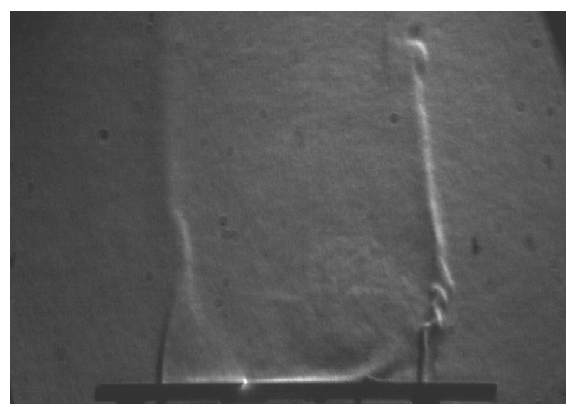

(e)

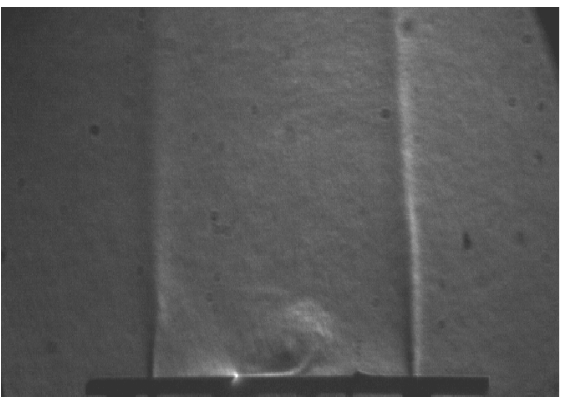

(c)

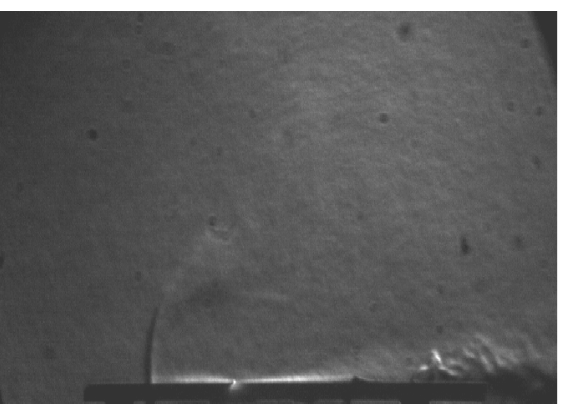

(f)

Fig. 23 Schlieren visualizations of the electric wind produced by a single-surface dielectric barrier discharge, at $t=0$ (a), $20 \mathrm{~ms}(\mathbf{b}), 40 \mathrm{~ms}(\mathbf{c})$, $80 \mathrm{~ms}(\mathbf{d}), 100 \mathrm{~ms}(\mathbf{e})$ and $600 \mathrm{~ms}$ (f) after the application of the AC high voltage. Here, $V_{\mathrm{AC}}=10.4 \mathrm{kV}$. From Moreau et al. (2008a)

Fig. 24 Starting vortex at $120 \mathrm{~ms}$ after discharge initiation (a) and velocity profiles of the electric wind produced in initial quiescent air, after the discharge switch on from 2 up to $400 \mathrm{~ms}$ $(x=5 \mathrm{~mm})$. a From Whalley and Choi (2010), b from Balcon et al. (2009a) (a)

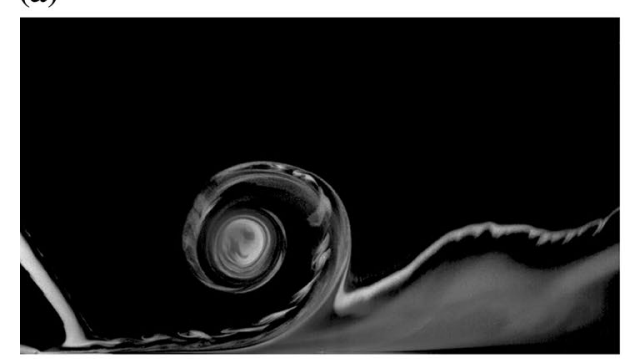

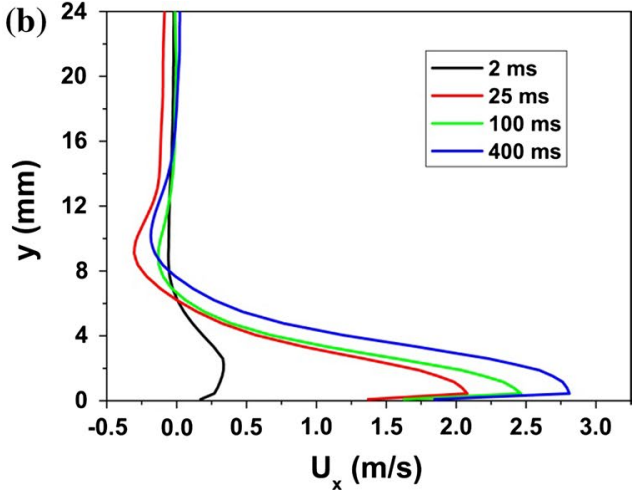

helium jets are deflected in the presence of electric wind. Jet (II) is deflected due to the flow produced by the discharge, tangentially to the wall. Jet (I) is deflected because the suction effect occurring above the plasma region.

Figure 23 shows the influence of the electric wind produced by a single DBD at several times, from $t=0$ (Fig. 23a) to $t=600 \mathrm{~ms}$ (Fig. 23f). At $t=600 \mathrm{~ms}$, the electric wind has reached its steady state. Just after the highvoltage application (and then the ignition of the surface discharge, at $t=0$ ), the electric wind starts to act, from the right edge of the air-exposed electrode toward the right, tangentially to the wall (Fig. 23b). This results in the formation of a starting vortex above the wall. Gradually, this vortex moves downstream in the direction of the produced electric wind (Fig. 23b-e). More recently, high-quality visualizations of this starting vortex have been published in Whalley and Choi (2010). In Fig. 24a, one of the visualization proposed in the reference (Whalley and Choi 2010) is shown. This image includes the large-scale starting vortex as well as a train of small-scale vortices that result from the flow fluctuations at the pulse frequency.

Consequently, deeper experiments have been performed by Balcon et al. (2009a). In this paper, the properties of the electric wind were investigated by spatially resolved and phase-locked PIV measurements. The goal was to evaluate the time required by the induced flow to reach a steady state. PIV flow fields showed that just after the discharge ignition, the interaction between the discharge and the surrounding air results in the formation of a counterclockwise rotating starting vortex located above the actuator. After 
(a)

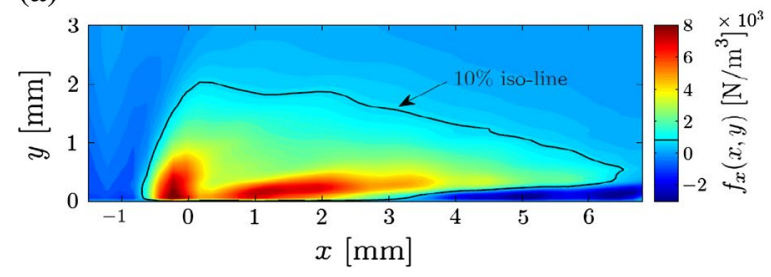

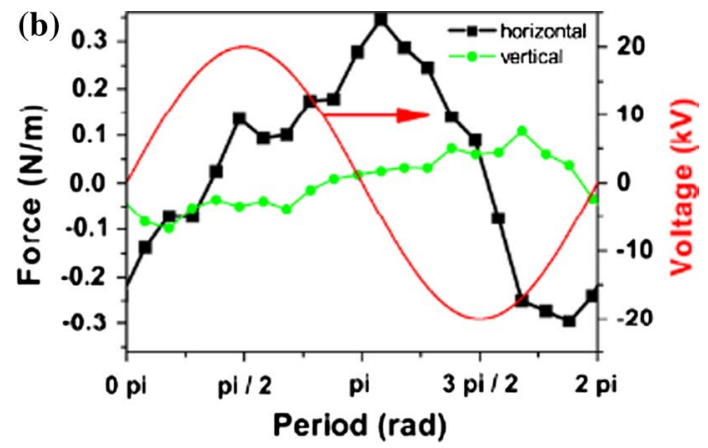

tor computed from PIV measurements (b). a From Kriegseis et al. (2012), b from Debien et al. (2012b, c)

the region in which the local force $f(x, y)$ is higher than the maximum force divided by ten. It highlights that, in the case of a 0.4-mm-thick dielectric with $V=12 \mathrm{kV}$ and $f_{\mathrm{AC}}=11 \mathrm{kHz}$, the local force can reach $8 \times 10^{3} \mathrm{~N} / \mathrm{m}^{3}$. This maximum force is located very close to the active electrode edge, typically at $x \approx 1 \mathrm{~mm}$ and $y \approx 0.15 \mathrm{~mm}$. However, the total computed force is equal to about $25 \mathrm{mN} / \mathrm{m}$ and the total measured thrust is around $15 \mathrm{mN} / \mathrm{m}$, the difference corresponding to the total drag due to viscous and pressure contributions (thrust is the sum of the EHD force plus the total drag).

The spatial distribution of the EHD force is of interest, but its temporal behavior is necessary if one wants to correctly implement surface plasma DBD in a turbulent flow solver. Among others, Enloe et al. (2009) estimate the total discharge thrust versus time with the help of a high bandwidth laser interferometer system (measures including the total drag of the plate supporting the actuator). They found that the negative-and positive-going half-cycles of the plasma discharge produce a force in the same direction, but they also showed that only the negative-going half-cycle produces a force sufficient to substantially overcome the drag induced by accelerating the air in the immediate vicinity of the wall. This scenario was numerically validated in Font et al. (2009) where results confirmed that the production of a net positive force for positive- and negative-going cycles of the discharge is the most probable scenario. Furthermore, it was shown that the wall viscous drag should increase when the plasma volume is reduced. This study numerically suggests that significant energy dissipation by viscous effect occurs during the $\mathrm{AC}$ cycle, as it was later demonstrated in Kriegseis et al. (2012).

In 2012, Debien et al. $(2012 b$, c) computed the timeresolved force imparted to the surrounding gas by a surface DBD actuator, from time-resolved velocity fields obtained by PIV measurements. The body force was calculated using the momentum equation in integral form inside a control 
(a)

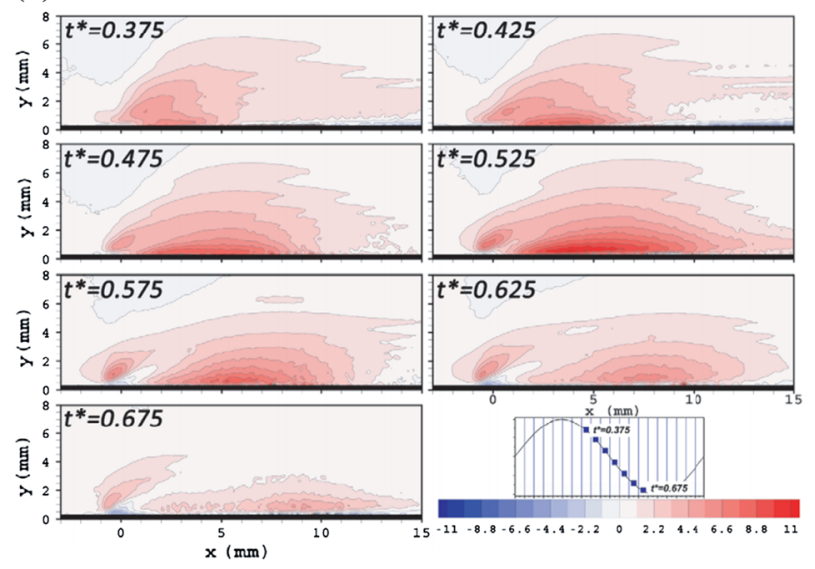

(b)

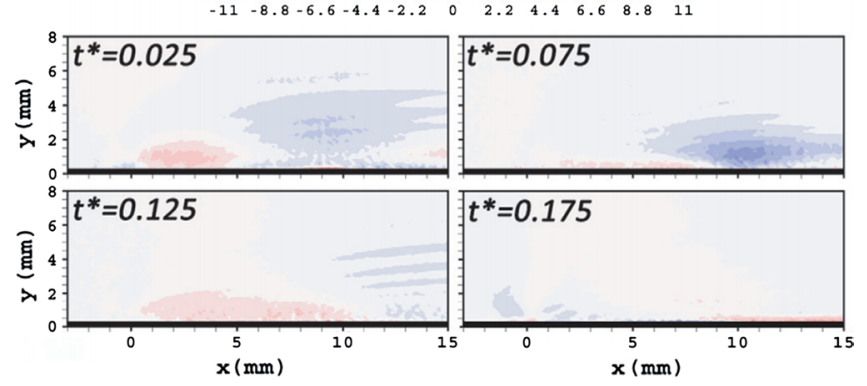

Fig. 26 Spatial distribution of the electrohydrodynamic volume force forms during a the negative-going cycle and $\mathbf{b}$ the positive-going cycle. Scale is given in $\times 10^{-3} \mathrm{Nm}^{-3}$. From Benard et al. (2013)

surface including the gas above the discharge. The time evolution of the EHD force computed by using this procedure is shown in Fig. 25b. The time-averaged horizontal EHD force is here equal to $34.2 \mathrm{mN} / \mathrm{m}$. The vertical force is smaller of about two orders of magnitude $(-0.37 \mathrm{mN} / \mathrm{m})$. One can see that the horizontal force is negative during the positive halfcycle and positive during the negative half-cycle (maximum and minimum values equal to 345 and $-293 \mathrm{mN} / \mathrm{m}$, respectively). The period of positive force is slightly longer than the negative force period, resulting in a positive time-averaged force. This result is nearly in agreement with numerical works published in Boeuf et al. (2009b) and Likhanskii et al. (2008), although the actuator design and the electrical parameters used in these studies were different than the one of Debien et al. (2012b, c). In these latter publications, measurements have been conducted for a $f_{\mathrm{AC}}$ frequency of $1 \mathrm{kHz}$. In 2013, similar results have been reported in case of actuator supplied by a $9.5 \mathrm{kHz}$ AC high voltage (Neumann et al. 2013). As in Debien et al. (2012b, c), the authors of Neumann et al. (2013) concluded that the produced flow results from a push/pull behavior for the EHD force, this in contradiction with the measurements and analysis of Enloe et al. (2009). In fact, the results obtained by using the methodology reported in Debien et al. (2012b, c) or Neumann et al. (2013) cannot be viewed as the pure behavior of the EHD force. Indeed, the total force is obtained by a spatial averaging of the local force over a constant domain. It is then considered that the EHD force occurs inside a constant domain regardless of the discharge phase. This is in contradiction with the visualizations of the plasma morphology that show significant variations in the plasma extension length versus time (see Figs. 4, 5). Furthermore, the method includes the convective part of the flow and the viscous turbulent losses that are indirectly caused by the discharge, but they do not contribute to the EHD force.
Finally, temporal and spatial time-resolved description of the EHD force has been proposed in Benard et al. (2013). The velocities of the electric wind are measured by a phase-averaged procedure achieving a temporal resolution of $50 \mu \mathrm{s}$ and a spatial resolution of about $100 \mu \mathrm{m}$. The distribution of force over the volume of gas is calculated from velocity measurements by resolving simplified Navier-Stokes equations where the pressure contribution is neglected (it is considered that the EHD force dominates the pressure gradient). The time-averaged total EHD force is in agreement with the literature (Debien et al. 2012b, c; Neumann et al. 2013), and the mean spatial distribution is similar to the topology reported in Kriegseis et al. (2012) (see Fig. 25a). Additionally to the mean spatial distribution, the time evolution of the EHD force is also described in Benard et al. (2013). As shown in Fig. 26a, the glow regime causes the production of a positive volume force, with a maximum positive volume force at the middle of the glow period. The positive-going cycle is also responsible for a positive volume force in the region where streamers propagate (Fig. 26b). However, the contribution of the streamer regime in the volume force at the origin of the electric wind production is weak by comparison with the volume force observed during the glow period (the momentum transfer is at least five times larger during the negative-going cycle of the applied voltage than during the positive-going cycle). These results support the push/push scenario developed from the measurements obtained by interferometry in Enloe et al. (2009). A significant negative volume force has also been observed during the AC period as in Debien et al. (2012b, c), Neumann et al. (2013). These negative volume forces form in periods where there is no discharge instead of an expected null value. In fact, in the absence of discharge the hypothesis that the EHD force dominates the pressure gradient is no more verified. The negative 

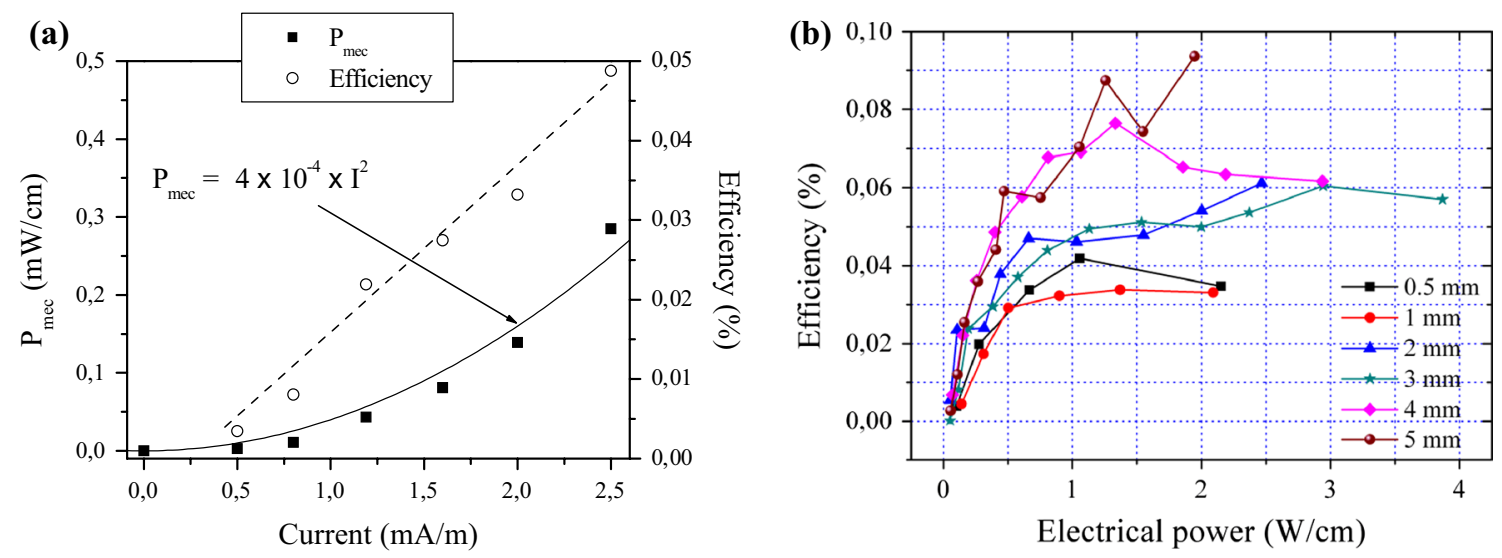

Fig. 27 Mechanical power and efficiency of a DBD actuator versus rms discharge current (a). Efficiency of a DBD actuator versus consumed electrical power for different dielectric thickness values (b). a From Moreau et al. (2005), b from Jolibois and Moreau (2009)

values are the signature of a strong positive pressure gradient caused by the fluid deceleration in the absence of EHD force. This deceleration originates from the viscous drag as initially proposed by Enloe et al. (2009), but Benard et al. (2013) shows that the turbulent energy dissipation resulting from the shear stresses between layers of the flow also contributes to an 'artificial' negative volume 'force.' Moreover, the domain in which the force is spatially integrated should be adapted to each phase of the discharge.

The agreement between total EHD force estimation by mechanical system (Enloe et al. 2009) and calculation from velocity measurements (Benard et al. 2013) suggests that the plasma discharge itself is responsible for two successive pushes over one voltage cycle. However, the major contribution comes from the glow regime period where the negative ions in the cloud at proximity of the air-exposed electrode are repelled downstream as proposed in Debien et al. (2012c). It is important to notice that force estimation by velocity measurement is an ongoing topic, and future developments will probably contribute to definitive conclusions on the spatiotemporal behavior of the EHD force.

\subsection{Efficiency of DBD plasma actuators}

As for all the electromechanical actuators, efficiency is a useful parameter. From velocity profiles such as in Fig. 12a, the induced kinetic power and then the electromechanical efficiency of such actuators may be computed. This is accurately explained in Moreau et al. (2005), Moreau (2007) and Moreau and Touchard (2008). Under the assumption of a stationary flow, the mechanical power corresponds to the kinetic energy density flow rate, which may be expressed by:

$P_{\text {mec }}=\frac{1}{2} \rho_{G} L \int_{0}^{\infty} U^{3}(y) \mathrm{d} y$ with $L$ the electrode length, $U(y)$ the velocity and $\rho_{G}$ the air density. Then, the electromechanical efficiency of the plasma actuator in the absence of free air stream is given by:

$\eta=\frac{P_{\mathrm{mec}}}{P_{\text {elec }}}$

Due to Eq. (7), the produced mechanical power is maximized at the extremity of the plasma extension, where the electric wind velocity is maximal. As illustrated by Fig. 27a, it seems that efficiency increases linearly with the discharge current (in this range, because for higher currents, there is an asymptotic behavior). More recently, Jolibois and Moreau (2009) computed plasma actuator efficiency versus electrical power consumption, for different dielectric thickness values (Fig. 27b). Several remarks can be done: (1) The efficiency value is very low, (2) efficiency increases with dielectric thickness, (3) efficiency increases for power consumption up to about $1.5 \mathrm{~W} / \mathrm{cm}$ and decreases when $P_{\text {elec }}>1.5 \mathrm{~W} / \mathrm{cm}$, (4) although the plasma actuator efficiency is very low, their effectiveness for flow control can be excellent because the flow control authority depends mainly on their ability to introduce small perturbations in the flow.

However, in these both papers, the grounded electrode was not encapsulated. With an encapsulated ground electrode, Laurentie et al. (2009) reached efficiency up to $0.2 \%$.

Another manner to compute plasma actuator efficiency is to use:

$P_{\text {mec }}=\iint_{x, y} f(x, y) \times U(x, y) \mathrm{d} x \mathrm{~d} y$

where the velocity field $U(x, y)$ can be determined by PIV measurements and the force distribution $f(x, y)$ can be 
computed from PIV data, as illustrated in the previous section. For instance, Giepman and Kotsonis (2011) use this method to compute the electromechanical efficiency and the time evolution of the mechanical power produced by a thin dielectric-based surface DBD. On the one hand, one can consider that their computation of efficiency is correct, as they are in agreement with previous computations. In fact, they confirm that the typical efficiency values were in the order of $0.1 \%$, with a maximum of $0.2 \%$. On the other hand, their computation of the mechanical power versus time is slightly biased because they assume that the force distribution does not change in time, as it is well known that the force is strongly unsteady. As a consequence, they find that the development of the produced power in time takes about $6 \mathrm{~ms}$ to reach a steady level. This is in contradiction with the results presented in section I where it has been demonstrated that the produced flow takes several hundred milliseconds to reach a steady behavior after the discharge initiation.

\section{Optimization of surface DBD for flow control}

In this second part of the paper, a survey of some geometrical, electrical and dielectric parameters that optimize the discharge in terms of produced force, induced electric wind or effectiveness is presented. This overview is voluntarily restricted to routes that have demonstrated a possible enhancement of the discharge effectiveness. Naturally, this list is not exhaustive, suggesting that others optimizations may exist but are not considered here.

\subsection{Influence of the electrode geometry and the dielectric properties}

\subsubsection{Influence of the active electrode}

As explained in the previous section, the plasma is formed from the edge of the air-exposed active electrode. The surface discharge in the gas results from the electric field due to the applied high voltage and its spatial distribution. The material used for the electrode of a DBD plasma actuator does not have a strong influence on its effectiveness. This has been verified in numerous studies without evidencing a clear enhancement of the momentum transfer (Hoskinson et al. 2009). However, a stronger electric field should increase the ionization rate and subsequently should affect the EHD force. A straight active electrode edge promotes an iso-potential distribution of the electric field, but a deformed electric field can be obtained by modification of the active electrode shape. This has been confirmed in Thomas et al. (2009) where the straight edge of the active electrode has been replaced by an electrode with a serrated

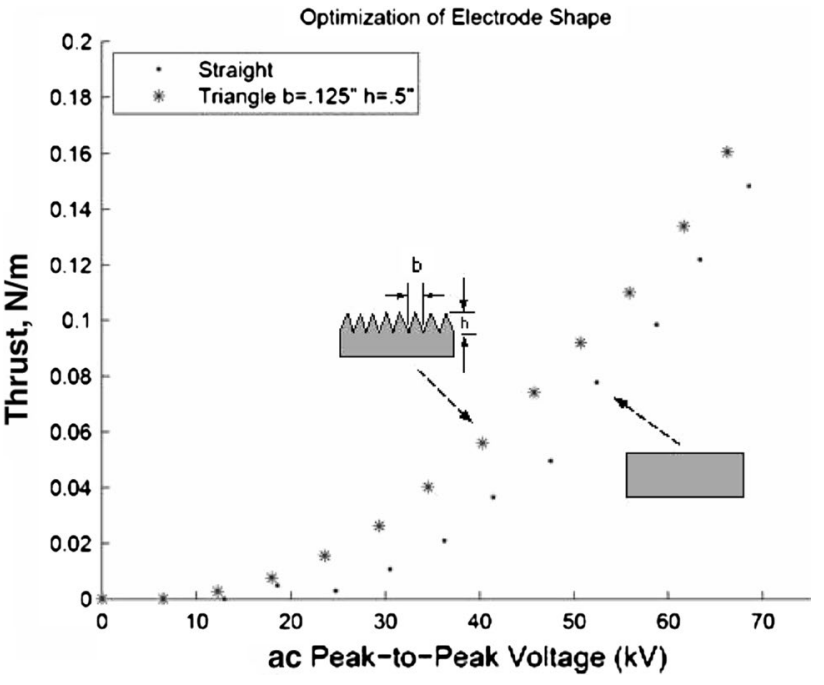

Fig. 28 Thrust per unit span in function the applied voltage for actuators with straight and serrated electrode. From Thomas et al. (2009)

downstream edge (triangular pattern). As a result of the promoted ionization rate and the earlier plasma ignition caused by the reinforcement of the electric field at the tips of the serrated electrode, this electrode design improves the thrust production from 5 to $400 \%$ (see Fig. 28). The measured thrust yields high amplitude due to a combination of favorable factors such as the use of a high-voltage amplitude, a low AC frequency, a thick dielectric material and an active electrode with a serrated design. The improvement is particularly spectacular at low applied voltage, but it decreases with the applied voltage. At the higher applied voltage, the reduction in thrust enhancement is due to the plasma sheet evolving in a filamentary regime. The increase in thrust production is evident, but the resulting electric wind was not documented in the paper. This was done in 2011, where PIV measurements have been conducted for linear and serrated electrodes types (Berendt et al. 2011b). The velocity profiles show that a serrated electrode leads to higher electric wind amplitude than the one measured with a linear active electrode. As in Thomas et al. (2009), the authors observe a reduction in the performance improvement when high-voltage amplitude is used. More recently, a detailed investigation has been proposed in Joussot et al. (2013) where electromechanical diagnostics are conducted. This paper reports that the filamentary and diffuse natures of the discharge over one AC cycle are not modified by using a serrated electrode. However, they observe that the streamers formed during the positive-going cycle propagate further with a serrated electrode, whereas intense corona spots occur at the tips of the serrated electrode. They confirmed the higher velocity production with a serrated electrode, and more specifically, they demonstrated that the produced velocity is maximized in the region in front of the 
electrode tips. By using a three-component LDV system, it was demonstrated that serrated electrode promotes a threedimensional flow topology leading to periodic vorticity regions along the edge of the serrated electrode. This production of vorticity was already observed in Durscher and Roy (2012) where a serpentine design was investigated. However, in Durscher and Roy (2012), the maximal mean flow velocity in the primary direction is reduced by comparison with a straight active electrode edge.

Another method for increasing locally the electric field consists of reducing the thickness of the active electrode. This was firstly proposed by Enloe et al. by investigating thin planar electrodes and wire electrodes with diameter ranging from 0.36 to $0.98 \mathrm{~mm}$ (Enloe et al. 2004b). They demonstrate that the plasma actuator effectiveness is influenced by the geometry of the active wire electrode. The power-to-thrust conversion is significantly greater when the thickness of the active electrode is reduced. The thrust improvement evolves linearly with the reduction in the electrode size, the maximal thrust being observed with a thin planar active electrode of $80 \mu \mathrm{m}$. The linear increase in thrust by reducing the electrode thickness has been recently contradicted in Hoskinson et al. (2008b, 2009, 2010). By extending the thickness range (from $1 \mathrm{~mm}$ down to $0.13 \mu \mathrm{m}$ ), they showed an exponentially increasing thrust when the diameter was reduced. In the thickness range investigated by Enloe et al. (2004b), the thrust increase is quasi-linear, but for very thin electrode (less than approximately $25 \mu \mathrm{m}$ ), the thrust production suddenly increases (see Fig. 29). This is associated with a modification of the discharge current. The use of a wire electrode of a few micrometers in diameter results in a reduction in the positive current peaks occurring during the streamer regime (Hoskinson and Hershkowitz 2010).

Recently, a deeper investigation has been conducted by Debien et al. (2012a) in order to characterize the effect of using a wire instead of a plate active electrode. In this study, the use of thin wires from 13 to $300 \mu \mathrm{m}$ in diameter as exposed electrode of a surface DBD plasma actuator has been experimentally investigated by electrical and optical diagnostics, electrohydrodynamic force measurements and produced electric wind characterization from time-averaged and time-resolved measurements. The first result concerns the discharge current behavior (Fig. 30). In the case of a conventional plate-to-plate $\mathrm{DBD}$, the positive-going cycle results in a streamer discharge (see the curves of the discharge current versus time, and the intensified CCD topview visualization during time windows $\mathrm{A}$ and $\mathrm{B}$ ). During the negative-going cycle, a glow discharge occurs as it is shown in the first part of this paper. This study confirmed that the amplitude of the positive current pulses caused by the streamer regime decreases with the wire diameter, finally resulting in a filament-free regime (as initially called

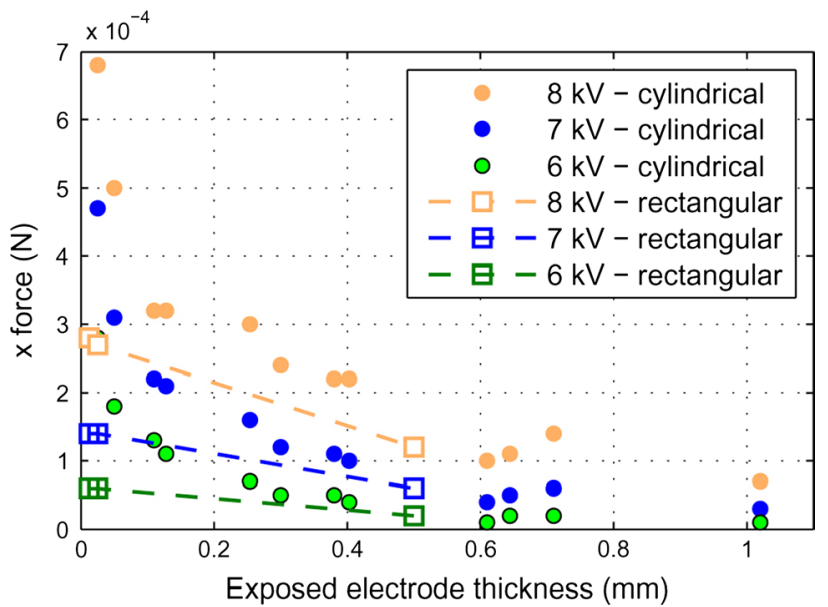

Fig. 29 Time-averaged forces induced by single barrier actuators with rectangular and cylindrical exposed electrodes. From Hoskinson et al. (2009)

in Hoskinson and Hershkowitz 2010) when the wire size is less than $25 \mu \mathrm{m}$ (see the current curve in Fig. 30 with a wire of $13 \mu \mathrm{m}$ in diameter). This is further illustrated by the fast-gated iCCD images where cancellation of streamers is clearly visible. Indeed, with the $13-\mu \mathrm{m}$-diameter wire, a filament-free corona discharge occurs during both going cycles.

The increase in thrust as a function of the electrical power consumption is shown in Fig. 31a. Thrust is a linear function of the electrical power consumption, regardless of the electrode geometry, in this range. However, the use of a thin wire active electrode instead of a plate one results in higher thrust for same electrical power consumption, suggesting an important increase in the actuator effectiveness. In fact, a gain in effectiveness of $46 \%$ is observed for a $13-\mu \mathrm{m}$ wire (maximum value of $0.97 \mathrm{mN} / \mathrm{W}$ ) (Debien et al. 2012a). This value is the best effectiveness value that has been measured up to now. When a thin wire is used as air-exposed electrode, Debien et al. (2012a) shown that the electromechanical conversion is increased. However, the most surprising result concerns the time evolution of the produced electric wind velocity. As illustrated by Fig. 32a, both going cycles are responsible for velocity production when only the negative-going cycle induces velocity in the case of a plate-to-plate DBD (see Figs. 18, 19, 21). The cancellation of the streamer regime results in two positive body force during the rising and falling part of the AC signal (Fig. 32b).

\subsubsection{Role and modification of the surface charges on the dielectric surface}

As it was shown in the first part of this paper, the EHD force scales with the electric field (see Eq. 5), then one 

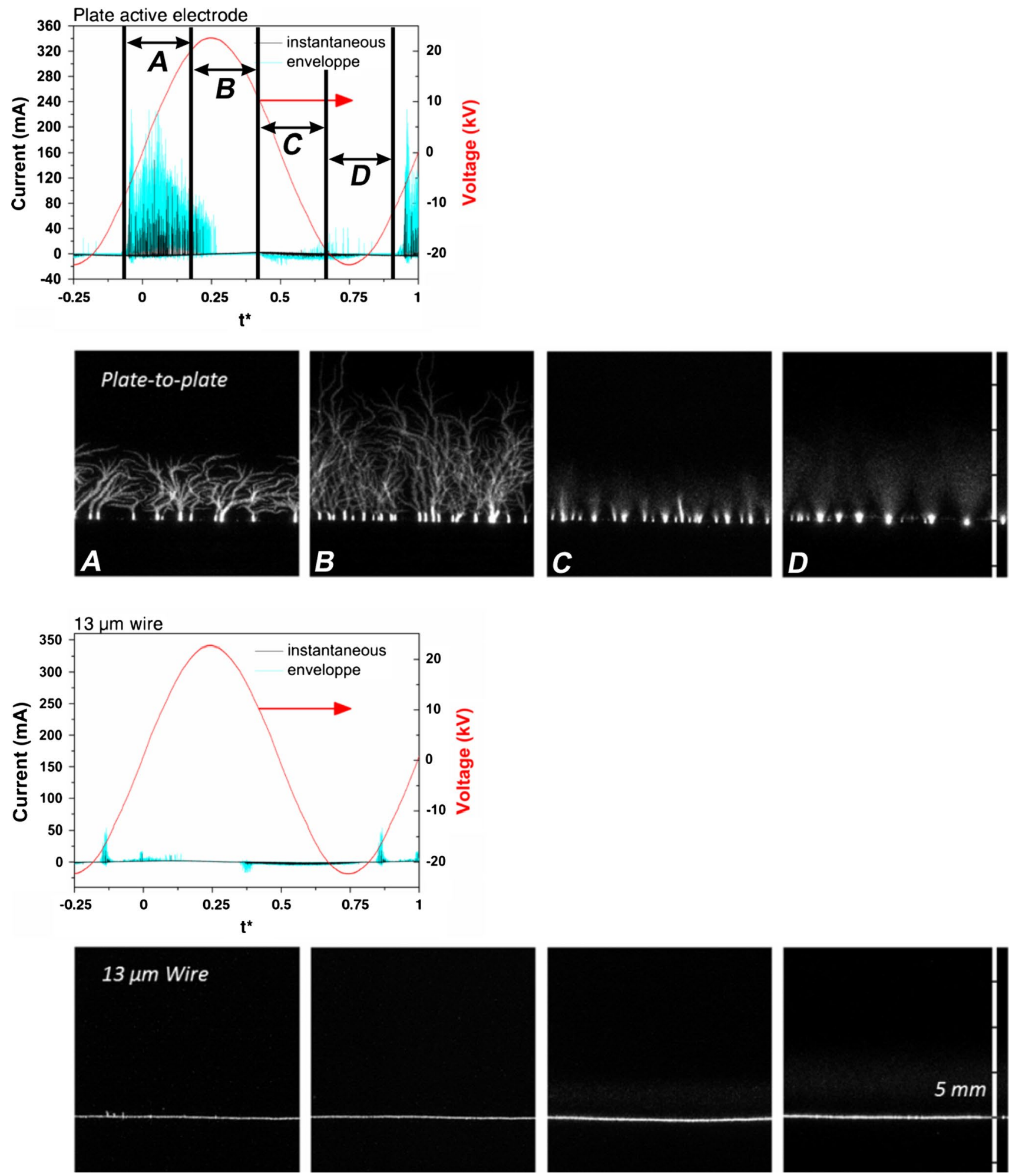

Fig. 30 Current versus time for a plate-to-plate DBD and $13-\mu \mathrm{m}$ wire-to-plate DBD (22 kV and $1.5 \mathrm{kHz})$, and top view of the plasma discharges (166 $\mu$ s exposure time obtained by iCCD). Normalized time $t^{*}=t \times f_{\mathrm{AC}}$ with $t$ the physical time and $f_{\mathrm{AC}}$ the high-voltage frequency, here $1.5 \mathrm{kHz}$. From Debien et al. (2012a) 


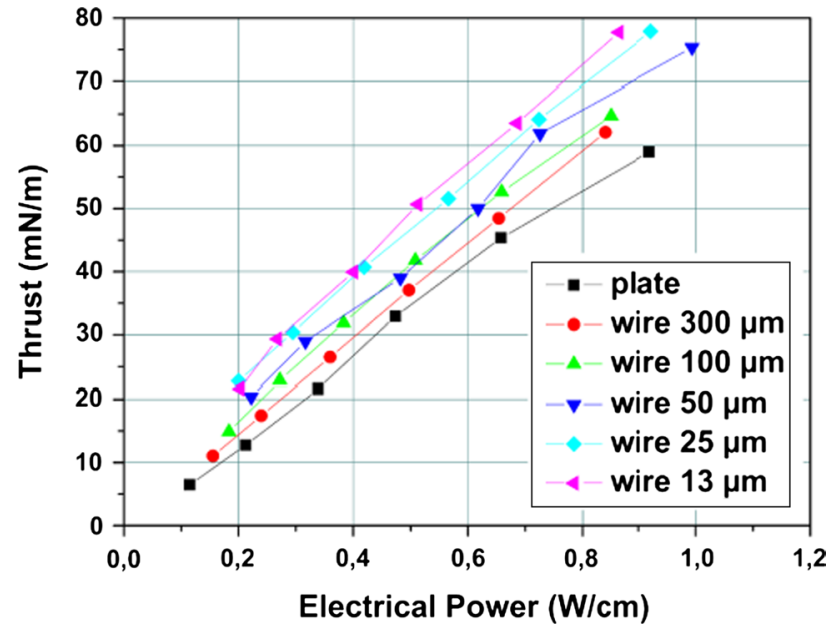

Fig. 31 Thrust versus electrical power (frequency $=1.5 \mathrm{kHz}$, voltages from 12 to $22 \mathrm{kV}$ ). From Debien et al. (2012a)

potential way for increasing the body force production consists of reducing the charge accumulation at the dielectric surface. Font et al. (2007) were among the first to interest in the potential amplitude and its spatial distribution on the dielectric surface of a plasma actuator (Fig. 33). Their results have been recently confirmed in Hong et al. (2013) where the time evolution of the total charge deposited on the dielectric surface have been quantified by capacitive measurements. Other methods such as laser polarimetry have been successfully used for measuring the time evolution of the surface charge density (Takeuchi et al. 2011).

Regarding the spatial distribution of the deposited charges, it was demonstrated in Font et al. (2007) that the surface charge increases monotonically as one moves downstream from the edge of the exposed electrode. In Takeuchi et al. (2011), it was observed that the amplitude of the charge fluctuations is more important close to the active electrode (see

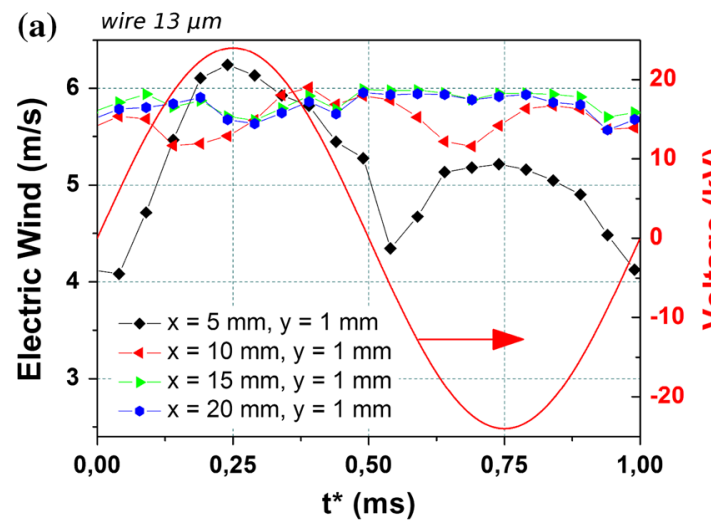

Fig. 32 Time evolution (PIV data) of a the horizontal electric wind velocity component for wire-to-plate actuator supplied by $24 \mathrm{kV}$ and $1 \mathrm{kHz}$ (from Debien et al. 2012a) and b the horizontal and vertical

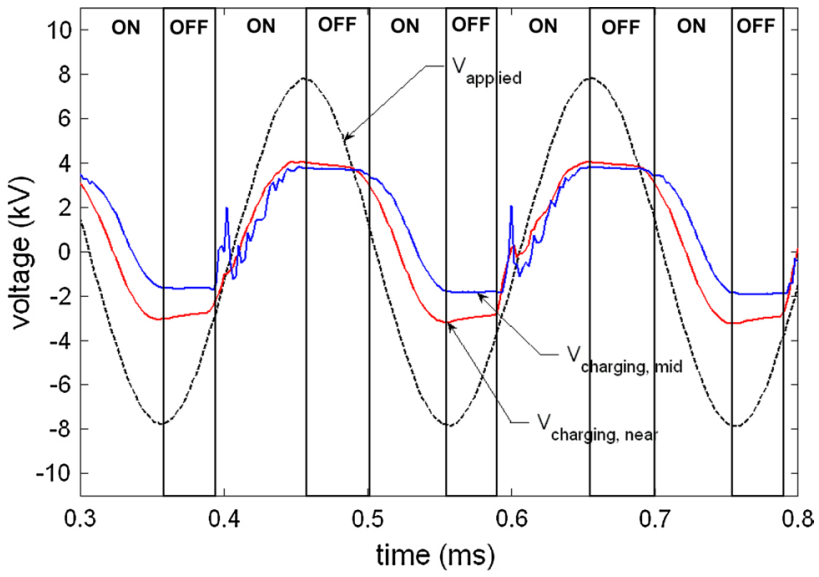

Fig. 33 Surface charging on the dielectric surface of a DBD by capacitive V-dot probe. From Font et al. (2007)

Fig. 34), but the mean charge value goes to an increasing DC value with the downstream distance in agreement with (Font et al. 2007). All the published results demonstrate that the surface of the dielectric barrier quickly presents a positive charge. Over one AC period, the surface charge density alternatively increases and decreases while charges remain nearly constant when the plasma discharge is quenched (Fig. 33). The surface potential can reach several $\mathrm{kV}$, a surface potential that can significantly modify the electric field produced by the voltage applied to the electrodes as confirmed in Takeuchi et al. (2011). With a thick dielectric, as illustrated by the current plot in Fig. 3a, the positive discharge occurring during the positive-going cycle starts when the applied voltage $V_{\mathrm{AC}}$ is equal to about $-10 \mathrm{kV}$, and the negative one ignites at about $+10 \mathrm{kV}$, meaning that the surface potential is higher than +10 and $-10 \mathrm{kV}$, respectively. The deposition of charges on the dielectric surface leads to a memory effect for the development of the successive discharges, and the

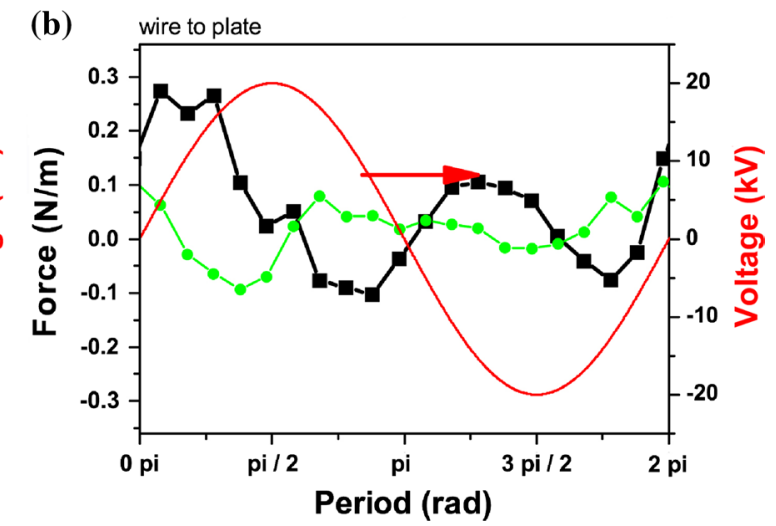

components of the produced force (calculated from velocity data) for actuator supplied by $20 \mathrm{kV}$ and $1 \mathrm{kHz}$. From Debien et al. (2012b, c) 


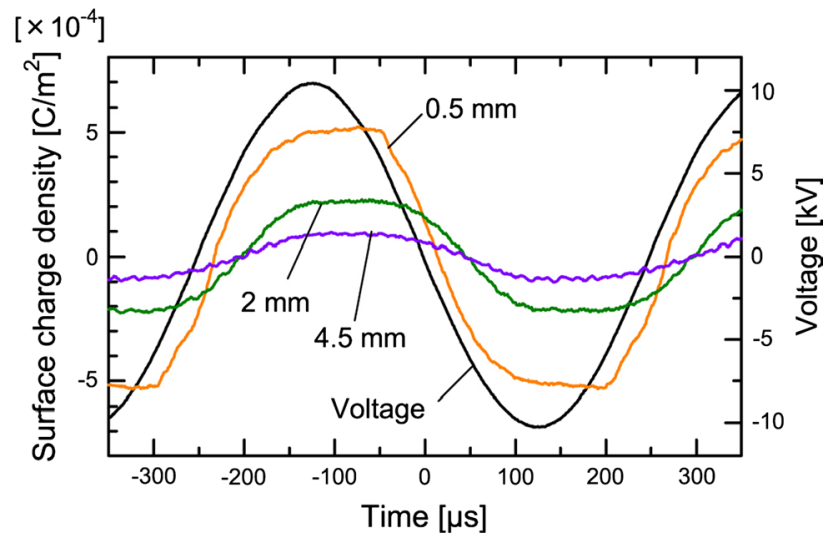

Fig. 34 Surface charging on the dielectric surface of a DBD by laser polarimetry. From Takeuchi et al. (2011)

stochastic behavior of the plasma formation is certainly governed by the charge deposited during the previous discharge as indicated by Zhu et al. (1995). More recently, the influence of the residual charges on the propagation of the streamer channels has been investigated (Deng et al. 2010). For pulse voltage, the residual charges are incriminated for modifications of the path of the streamer channels and branching as well as for modifications of the propagation velocity of the streamer channels. Among the obtained results, it was shown that residual charges of opposite polarity promote faster streamer propagation.

An easy way for modifying the charge deposited on the dielectric barrier is the use of materials having different permittivity (or dielectric constant) $\varepsilon$. In 2006, Roth and Dai provided an exhaustive parametric study for the optimization of plasma actuators (Roth and Dai 2006). Among the investigated parameters, twelve different dielectric materials have been considered, with material permittivity ranging from 3 up to 10 (see Fig. 35). Unfortunately, results for only two materials are proposed in the paper (Teflon and quartz). The velocity measurements indicate that a higher dielectric constant enhances the momentum transfer at the price of an electrical overconsumption. This was the first evidence that the use of a material that can reduce the charges on the dielectric surface (i.e., a large $\varepsilon$ value) is a way for improving the momentum transfer of the actuator. In 2007, the role of the dielectric material was examined by Pons et al. (2007). As it is indicated in this paper, the consumed power increases linearly with the relative permittivity and the maximal electric wind velocity also vary like the permittivity of the material. In Forte et al. (2006), velocity measurements for glass $\left(\varepsilon_{r}=10\right)$ and PMMA $\left(\varepsilon_{r}=3\right)$ materials are compared. The results lead to similar conclusions. At a fixed voltage, a material with a high permittivity produces higher velocity than with a material having a low dielectric constant but, for a fixed consumed power, low permittivity materials are more efficient in terms of power-to-velocity conversion. By increasing the permittivity, polarization effects are enhanced that finally reduces the charges deposited on the wall surface. Furthermore, the porosity of the material promotes transition to a filamentary regime if a too high-voltage amplitude (or AC frequency) is used (Forte et al. 2006; Thomas et al. 2009). In fact, the surface discharge occurring at the dielectric wall depends strongly on the physicochemical properties of the dielectric surface and on how the material interacts with the discharge. For instance, the behavior of a surface DBD is affected by the hydrophobic properties of a dielectric.

Beyond simply using materials with different dielectric constant, a few authors looked at more advanced methods for 'controlling' the residual surface charges. Opaits et al. $(2008,2009)$ proposed a DBD geometry where the dielectric covers only a part of the grounded electrode, leaving the rest of the electrode exposed (Fig. 36a). This configuration was supported by preliminary measurements of the residual charges with an electrostatic voltmeter. They observed that the residual surface charges build up far downstream from the air-exposed electrode. As the dielectric covers only the region at the immediate proximity of the air-exposed electrode, charges are supposed to be collected by the air-exposed part of the grounded electrodes. Unfortunately, only preliminary results are introduced in Opaits et al. (2008, 2009). The authors confirmed that the surface charge has been substantially reduced by exposing the electrode, but the benefit in terms of produced momentum transfer remains to be confirmed.

A solution to reduce the charge accumulation at the dielectric surface consists of using a coating film. For instance, semiconductive amorphous silicon was used to increase the charge mobility. In Guo et al. (2009), a thin film of a-Si (dielectric constant about 11) was deposited on the top of the dielectric surface. In addition, a second grounded electrode was put over the conductive thin film layer (see Fig. 36b). The objective of the authors was draining the surface charge through the semi-conductive film acting as a resistive material. The third electrode is used to collect the charges accelerated by a faster diffusion caused by the a-Si layer. The results in terms of thrust production show a small improvement of the momentum transfer with a maximal thrust gain roughly equal to $1.5 \mathrm{mN} / \mathrm{m}$, this for a nominal thrust of $9 \mathrm{mN} / \mathrm{m}$. By using the photoelectric characteristic of the thin film, the authors demonstrate that the thrust is increased with the surface conductivity (see Fig. 37). High permittivity material has also been tested recently. Fine and Brickner (2010) coated the surface of an alumina ceramic plate with a $\mathrm{TiO}_{2}$ layer (dielectric constant about 80-100). The electrode arrangement (Fig. 36c) and the coating film conduce to a significant increase in the produced thrust especially at low voltage where increase of about $120 \%$ is observed (nominal thrust of $2 \mathrm{mN} / \mathrm{m}$ ). At higher voltage $\left(6.5 \mathrm{kV}_{\mathrm{rms}}\right)$, the nominal measured thrust is $10 \mathrm{mN} / \mathrm{m}$ but 
Fig. 35 Dielectric constant of a variety of materials and measured electric wind production and consumed power for quartz and Teflon dielectric materials. From Roth and Dai (2006)

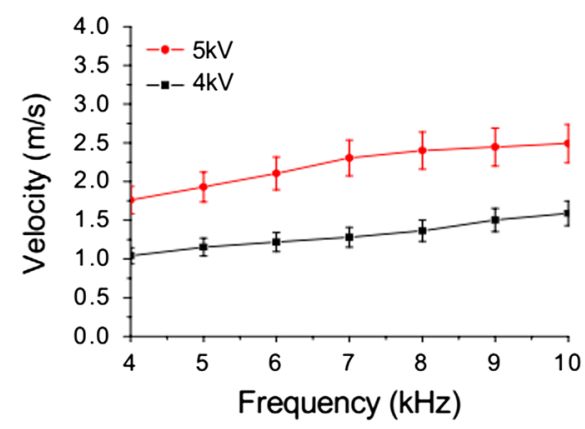

(a) Teflon actuator

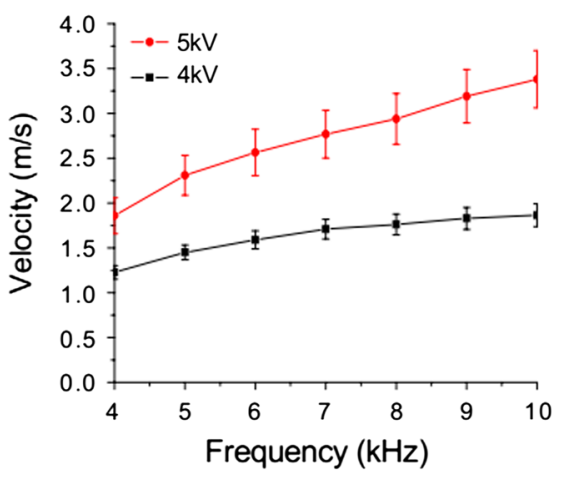

(c) Quartz actuator

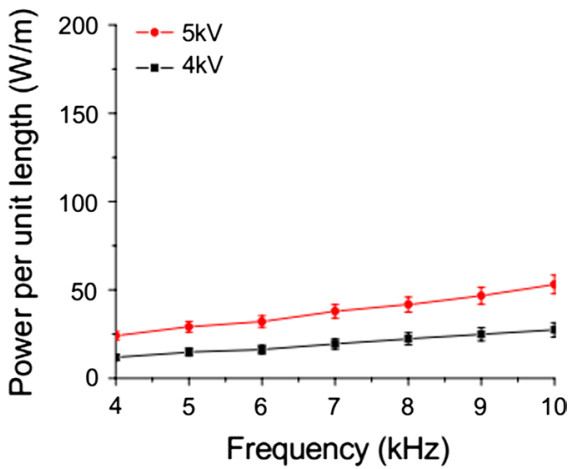

(b) Teflon actuator.

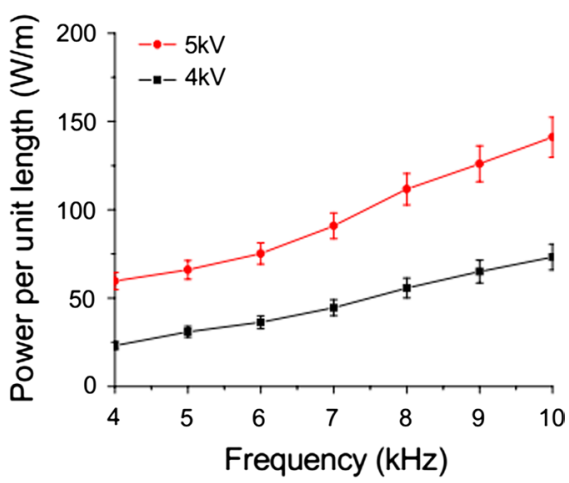

(d) Quartz actuator

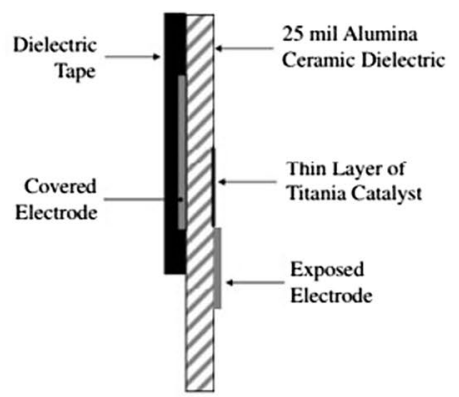

(c)

(a)

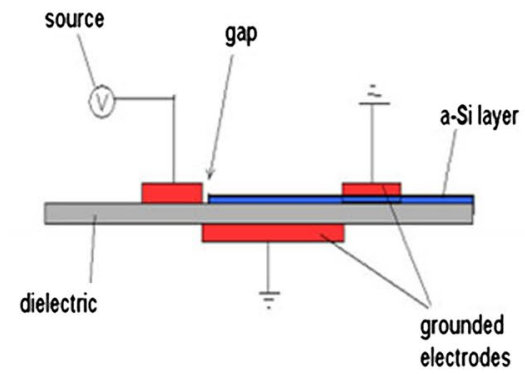

(b)

Fig. 36 Different electrodes geometry for modification of the surface properties regarding the residual charges. From a Opaits et al. (2008), b Guo et al. (2009) and c Fine and Brickner (2010) 


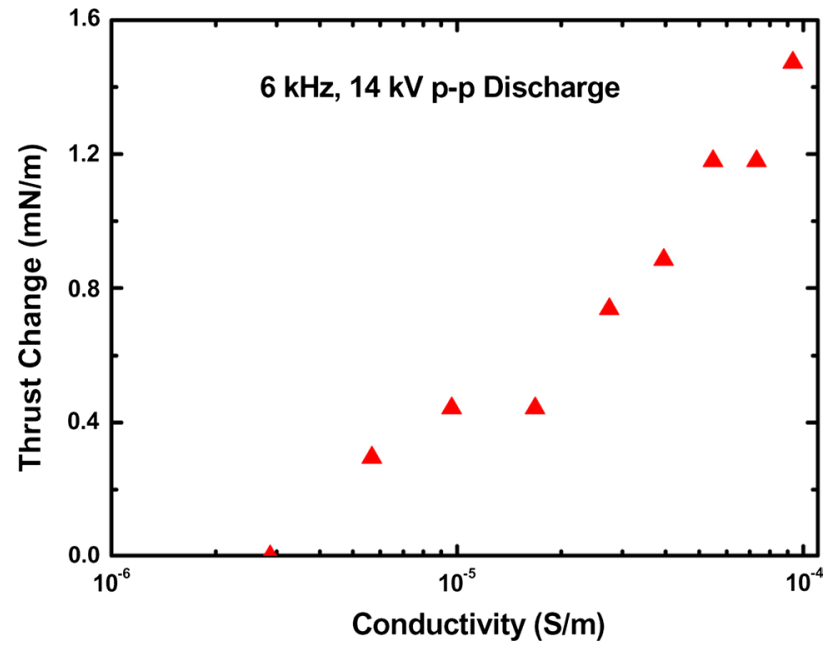

Fig. 37 Thrust improvement versus the conductivity of the dielectric barrier. From Guo et al. (2009)

the coating film with a high permittivity material causes an improvement of $40 \%$ on the produced thrust.

Linen-based phenolic coating, zinc oxide $(\mathrm{ZnO})$ plates (Opaits et al. 2009), silicon and carbon coating were used by Opaits et al. for increasing the conductivity of the dielectric surface. The zinc oxide plate has shown many drawbacks regarding its use as dielectric barrier in DBD configuration, the main one being a too high conductivity that leads to difficulties for ignition of the discharge. Linenbased phenolic has also a too small resistivity, and thrust measurements do not reveal a real improvement of the discharge effectiveness by comparison with a typical DBD. In Opaits (2012), coating based on silicon and carbon has been applied on a 2-mm-thick glass plate. The produced thrust when a voltage pulse is applied has been measured. Results show that the carbon coating has a lower efficiency compare to the silicon one. Furthermore, carbon coating promotes the formation of long filaments at the dielectric surface, these filaments sometimes evolving into a short circuit. In 2013, Starikovskiy and Miles have developed and studied an active surface composed of diode arrays that conduct the current in one direction and block the backward current to avoid the backward discharge (Starikovskiy and Miles 2013). Semi-conductive substrate is etched and doped by ion implantation. This dielectric was investigated for discharge supplied by a pulse of voltage ( $25 \mathrm{~ns}$ pulse duration). The effectiveness of the diode surface is estimated by applying pulses of different polarities. Experiments at low pressure (5 Torr) have confirmed the capability of this diode surface for cancelling the discharge usually produced by a positive pulse. This study show promising perspectives for cancelling chosen plasma regime.

According to the plasma physics and the importance of the spatial distribution of both the charged particle densities and the electric field, it is highly expected that modification of the charge accumulation can largely improve momentum transfer in surface DBD. However, this interesting option was not deeply investigated and the available studies did not show spectacular enhancement of the discharge performance. Indeed, in most of these studies, an improvement of the performance is observed, but this improvement is weak by comparison with a DBD actuator operating over a thick dielectric and at high-voltage amplitude (sees Section I where thrust up to $100 \mathrm{mN} / \mathrm{m}$ is reached). The interaction of plasma and surface is a key point for improving performances, but lot of work remains to find dielectric material suitable for draining surface charges without promoting transition to a filamentary regime.

\subsection{Influence of the electrical input signal}

\subsubsection{Effects of the electrical waveform on the time-averaged mechanical characteristics}

As the force production relates to the discharge regimes, a change in the distribution of streamer and glow regimes may affect the resulting mean and fluctuating body force. This can be easily verified by applying different types of input waveform HV. As it is illustrated in Fig. 38, streamer and glow regimes can be modified accordingly to the applied waveform. The duration of these regimes and the density of the current peaks can be varied. For instance, the period of a glow regime can be adjusted from 10 to $50 \%$ of the discharge period by using a square or negative sawtooth, respectively.

Investigations on the influence of the electrical waveform on the produced mean thrust start in 2004 with the paper published by Van Dyken et al. (2004). They compared the thrust produced by sine, square, triangular and positive-/negative-sawtooth waveforms, and they concluded that the thrust production at constant consumed power is maximized when using a negative sawtooth. The same year, Enloe et al. (2004a) published comparative results for positive and negative sawtooths. As depicted in Fig. 39, they confirmed that there is an increase in the thrust production for a negative-sawtooth signal. It was concluded that the thrust enhancement is due to more diffuse plasma for a greater fraction of the discharge cycle when negative sawtooth is applied. Abe et al. (2007) studied the influence of 10 waveforms on the thrust produced by a surface plasma developing over a glass-fiber-reinforced-epoxy dielectric barrier. They observe that a negative sawtooth improves the thrust by comparison with a positive one, this being in agreement with the results from Enloe et al. (2004a). However, the larger thrust reported in Abe et al. (2007) corresponds to a sinusoidal input waveform, this contradicting with results from Van Dyken et al. (2004). 

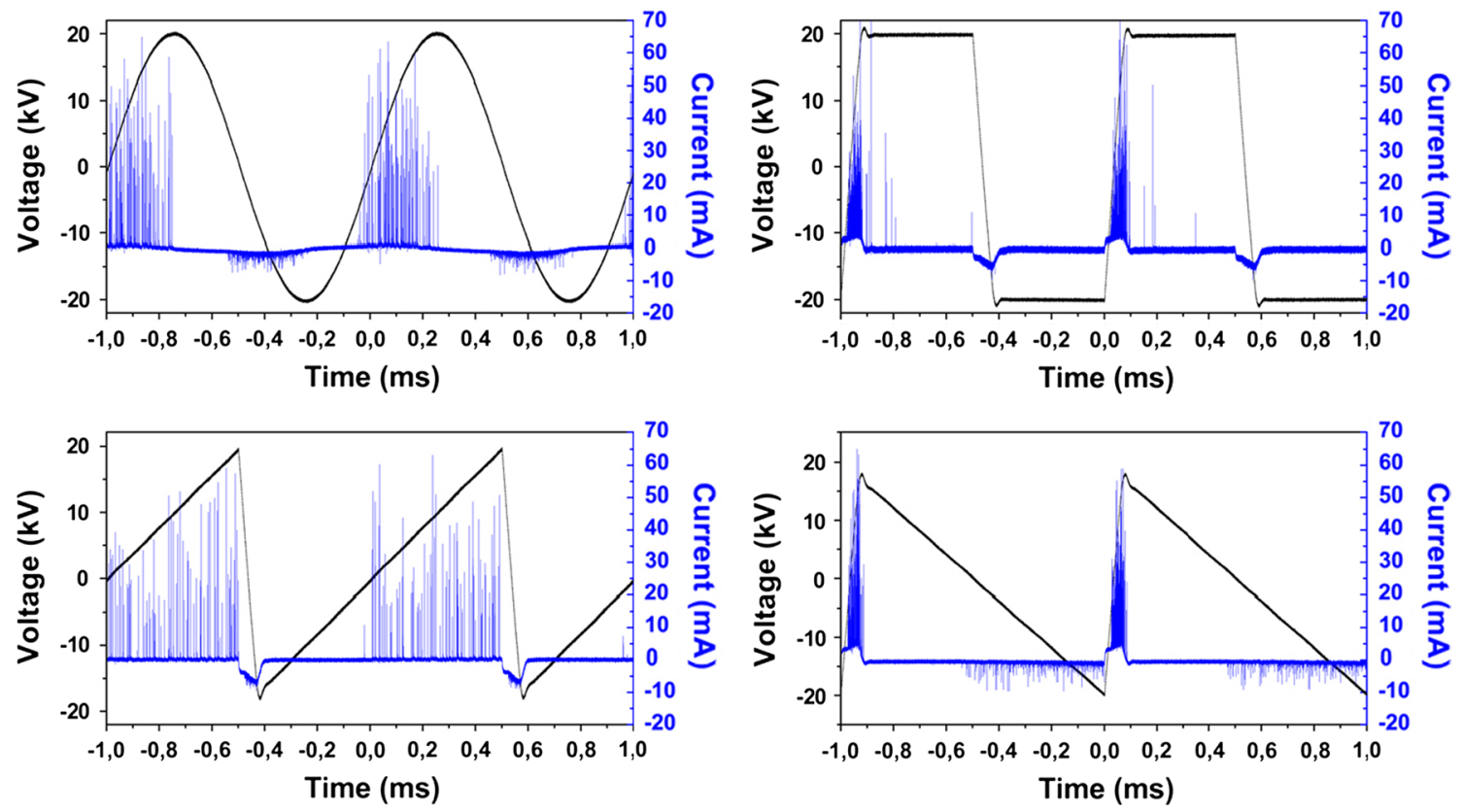

Fig. 38 Discharge current for four types of applied waveforms for DBD over 3-mm-thick PMMA dielectric. From Benard and Moreau (2012)

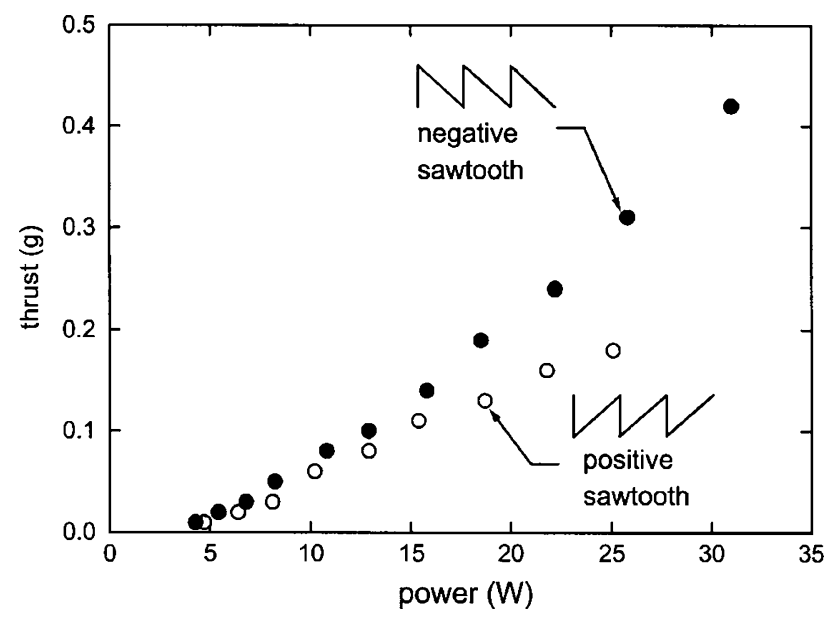

Fig. 39 Thrust versus dissipated power for both positive- and negative-sawtooth voltage waveforms applied to the plasma actuator (from Enloe et al. 2004a), and waveforms have been recalled to match with the terminology used in the paper)

Recently, in Benard and Moreau (2012), direct thrust measurements also show that symmetric waveforms (square and sine waves) produces a larger mean thrust compared to asymmetric waveforms (positive and negative sawtooths) as illustrated in Fig. 40. If one wants to optimally use DBD actuators in terms of mean force by electrical power consumption, sine waveform as input voltage is recommended in Benard and Moreau (2012). Indeed, a sine waveform gives the best performance for a given consumed electrical power. However, if the objective is a maximization of

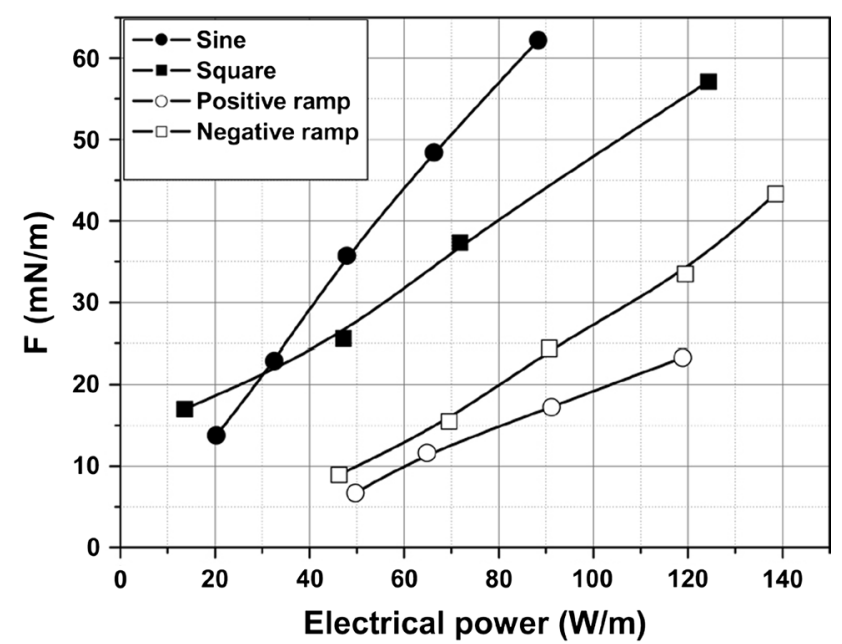

Fig. 40 Thrust produced by the DBD discharge versus the consumed electrical power. From Benard and Moreau (2012)

the produced thrust without consideration for the consumed power, a square signal input is better.

It is important to notice that the mechanical quantity measured to characterize the performances of a surface DBD actuator can lead to different conclusions. For instance, local velocity measurements by using a pressure probe indicate that the maximal induced velocity versus the consumed power is not really dependent on the applied waveform (Fig. 41). However, all the results follow an asymptotic behavior showing the effect of saturation at power consumption higher than $2 \mathrm{~W} / \mathrm{cm}$. In fact, the applied 


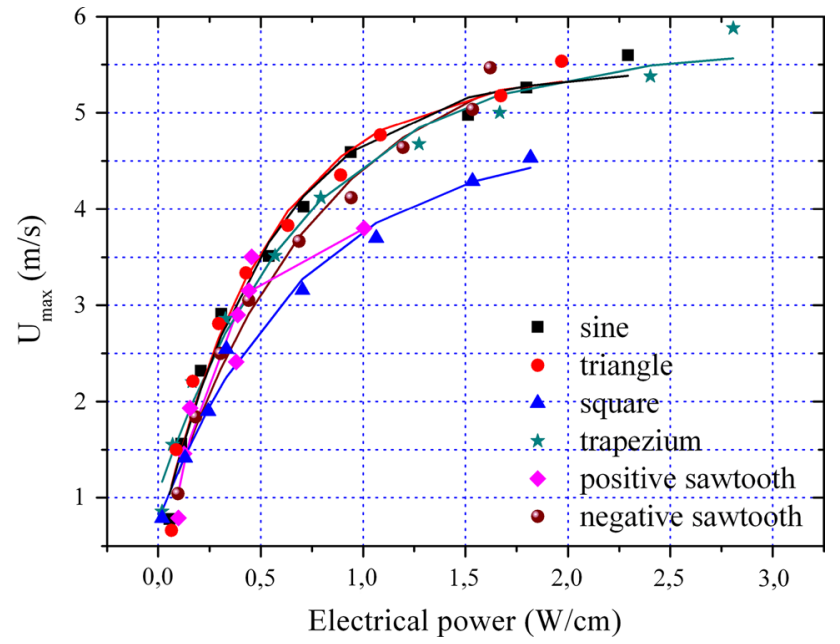

Fig. 41 Maximum electric wind for a variety of input waveform signals. From Jolibois and Moreau (2009)

waveform slightly influences the maximum velocity, but the used HV waveform affects primarily the thickness of the electric wind profile. This can be observed from timeaveraged velocity field measurements for a positive and a negative sawtooth. In Balcon et al. (2009b), an experimental campaign using PIV measurements demonstrates the modifications in the flow topology for positive and negative sawtooths (see Fig. 42). On the one hand, the positive sawtooth leads to a thick wall jet with suction region covering the region of plasma discharge. On the other hand, the negative-sawtooth waveform voltage results in a more homogenous velocity distribution in the boundary layer region with a velocity gain of about $25 \%$ on a localized region above the surface compared to the electric wind measured from a positive sawtooth input signal. It is clear that the significant difference in the flow topology will produce two different thrust values as measured in several publications.

\subsubsection{Effect of the electrical waveform on the time history of the mechanical characteristics}

Quantitative optical diagnostic such as laser Doppler velocimetry (LDV) can provide a localized time-resolved view of the induced flow field (Benard and Moreau 2012; Kotsonis and Ghaemi 2011). Results presented in Fig. 43 clearly show that periods of $U$ velocity production (horizontal velocity) correspond to the negative-going cycles of the applied HV, whatever the HV waveform. In contrast, the $U$ velocity decreases during the streamer discharge occurring in the positive-going cycle of the discharge. It results that the $U$ horizontal velocity component is a mirror of the input electrical waveform. As a result, the flow fluctuates at the same frequency than the high-voltage waveform as it was already shown in Forte et al. (2006, 2007) for a sine waveform. In Fig. 44, flow acceleration due to plasma is introduced (data from Kotsonis and Ghaemi 2011). As indicated in this plot showing the flow response to a square waveform input, a steeper negative-going period enhances the flow acceleration but over a shortened period. The variations in the flow accelerations result in fluctuating velocities. In Fig. 43, the larger fluctuations in the $U$ velocity component are observed for a sine waveform when they are minimized by using a positive ramp ( 1 and $0.5 \mathrm{~m} / \mathrm{s}$, respectively). This suggests that sine waveform is probably more effective in flow control strategies based on unsteady forcing at the driven $\mathrm{AC}$ frequency. The behavior of the vertical velocity component $V$ is also resolved by LDV measurements. For most of the investigated waveforms, this component remains negative indicating that the mean flow is deviated toward the
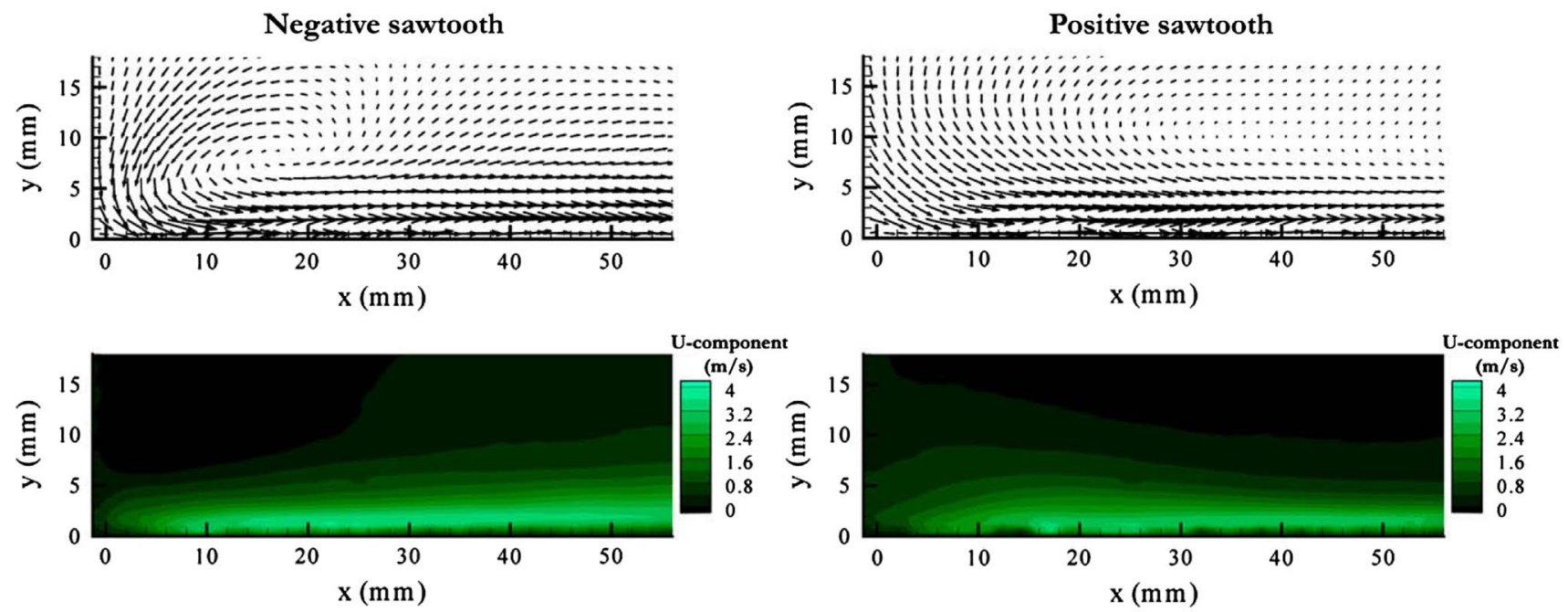

Fig. 42 Flow topology for both positive- and negative-sawtooth voltage waveforms applied to the plasma actuator. From Balcon et al. (2009b) 

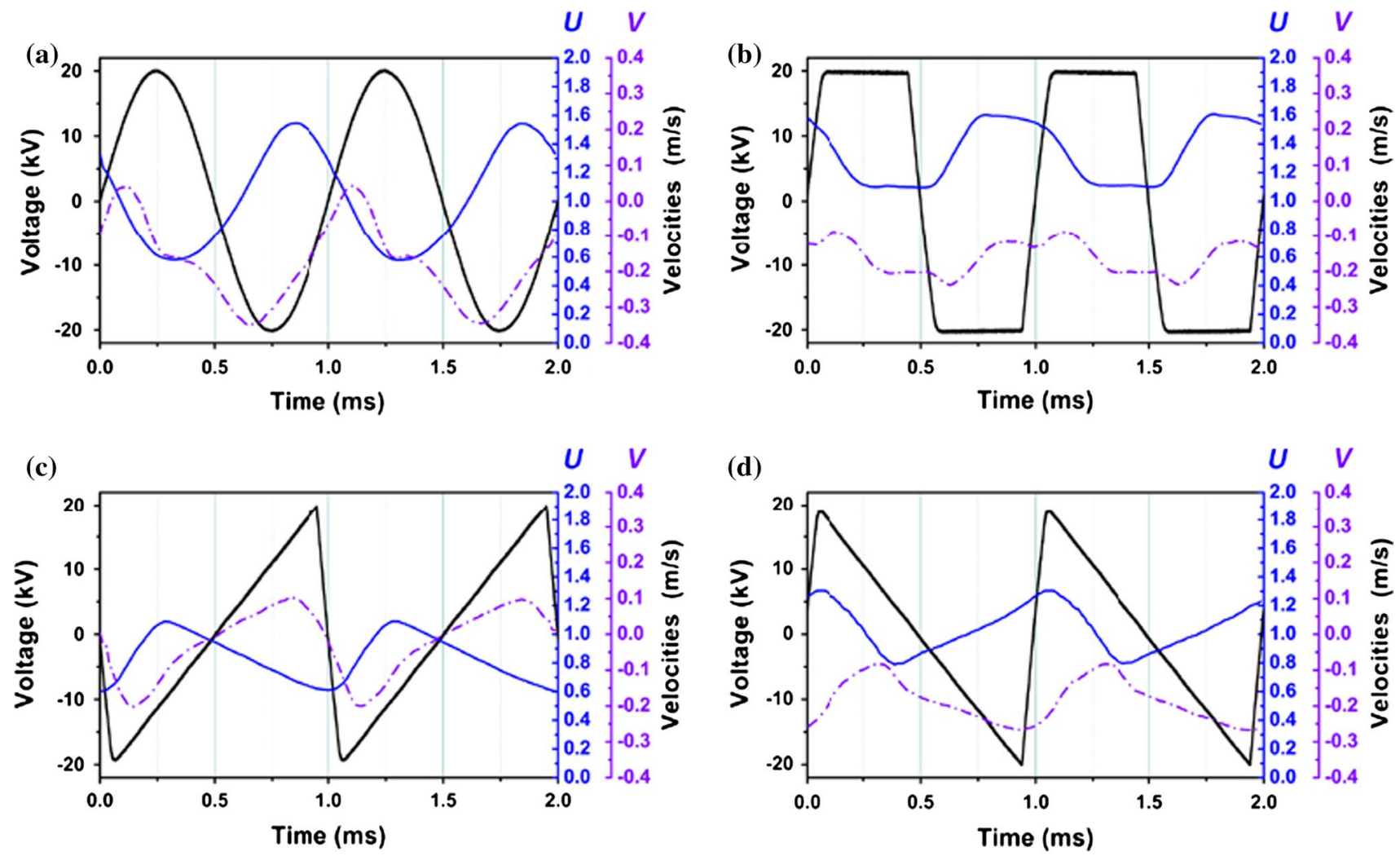

Fig. 43 Time-resolved electric wind $U$ (horizontal, i.e., in the plasma layer direction) and $V$ (normal direction) velocity for a a sine waveform input, $\mathbf{b}$ a square waveform, $\mathbf{c}$ a positive ramp voltage and $\mathbf{d}$ a negative ramp voltage. Measurements at $5 \mathrm{~mm}$ from the edge of the

active electrode and $1 \mathrm{~mm}$ above the dielectric surface for applied voltage amplitude of $18 \mathrm{kV}$ and frequency of $1,000 \mathrm{~Hz}$ (i.e., non-constant consumed electrical power). From Benard and Moreau (2012)
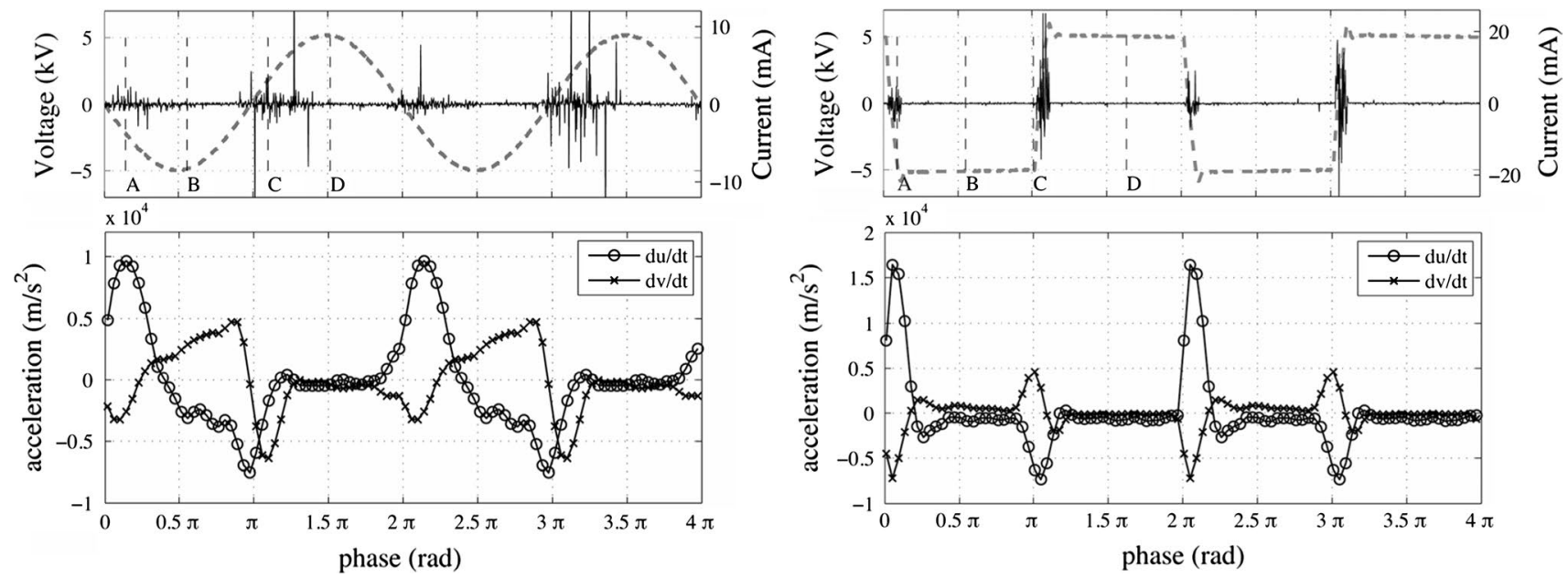

Fig. 44 Temporal evolution of acceleration for sine and square waveforms (values from PIV probed at 2 mm from the edge of the active electrode and $0.2 \mathrm{~mm}$ above the dielectric surface). From Kotsonis and Ghaemi (2011)

dielectric wall. In contrast, for a positive ramp some periods of the time history of $V$ exhibit positive values indicating that flow can be repealed from the wall in agreement with the thickening of the induced flow shown in Fig. 42.

\subsubsection{Toward an optimized waveform}

Logically, the strong relationship between the applied voltage and the resulting electric wind suggests that 

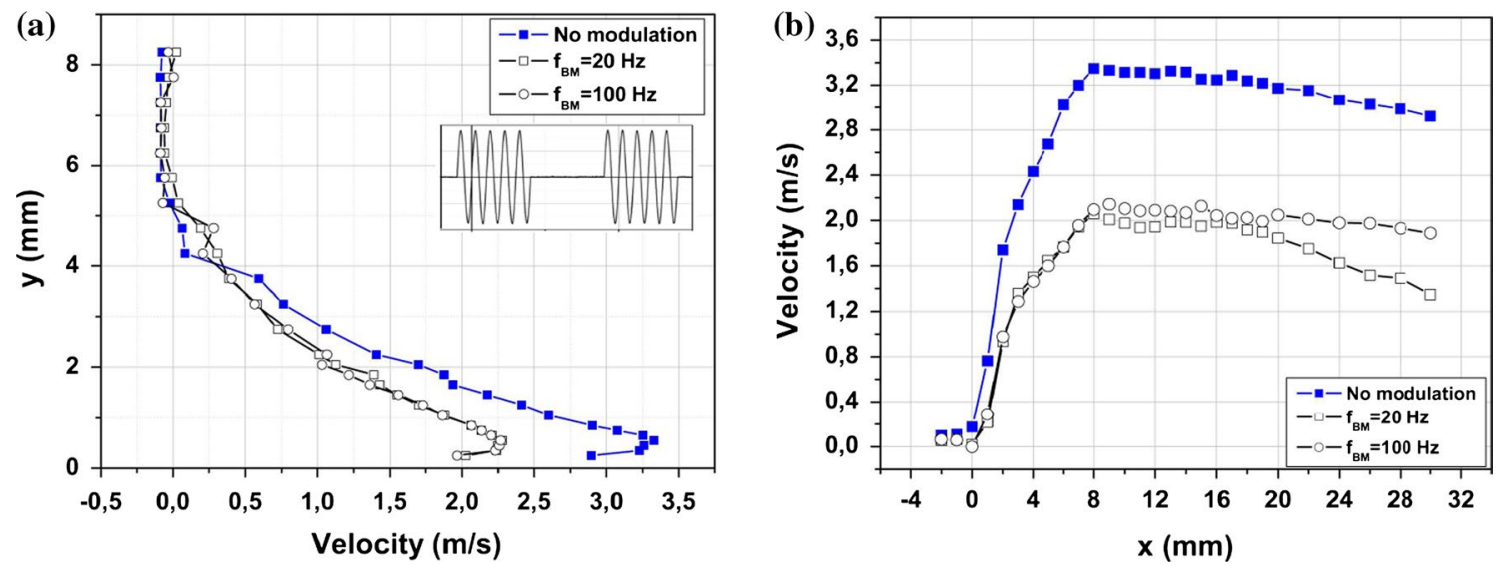

Fig. 45 Time-averaged velocity profiles (LDV measurements) along a the $y$ direction (at $x=9 \mathrm{~mm}$ ) and $\mathbf{b}$ the $x$ direction (at $y=0.25 \mathrm{~mm}$ ) for $f_{\mathrm{AC}}=1 \mathrm{kHz}\left(\right.$ at $18 \mathrm{kV}_{\mathrm{amp}}$ ) and $f_{\mathrm{BM}}$ equal to 20 and $100 \mathrm{~Hz}$ with duty-cycle of $50 \%$. Adapted from Benard and Moreau (2009)

optimization of the waveform is a way to increase the actuator performances. This was investigated in Abe et al. (2007). For instance by reducing the negative and positive component of a sinusoidal signal, they observe a large reduction in the thrust production by comparison with a typical sine waveform. They conclude that both negative and positive parts of a sine waveform are fundamental for an optimized net momentum transfer. Positive or negative half period alone is not sufficient to produce significant momentum transfer probably due to the importance of the charges deposited on the dielectric surface for the EHD force production mechanism. The authors also propose as electrical input a sine wave where the slope of the negative-going cycle has been increased. This modification shows a small benefit for the produced thrust $(+10 \%)$. In Kotsonis and Ghaemi (2012), the authors look at the effects of combinations of sine and square waveforms on the produced thrust and electric wind velocity. They define an optimal waveform that can improve the efficiency by $30 \%$ by comparison with a same actuator operated by a sine input waveform. The improvement in thrust production is also significant $(+45 \%)$, and a sine waveform produces about $40 \mathrm{mN} / \mathrm{m}$ when the optimized waveform reaches $58 \mathrm{mN} / \mathrm{m}$.

Optimizing the electrical waveform is a promising approach, but up to now no remarkable improvement has been achieved. Indeed, mostly to reduce the risk of dielectric failure or due to the amplifier specifications, the parametric studies available in the literature have been performed at low-voltage amplitude or AC frequency. It results that despite the improvement presented in these papers, higher thrust and electric wind velocity values are achieved with a single plate-to-plate DBD supplied by a sine high voltage (Forte et al. 2006; Debien et al. 2012a; Thomas et al. 2009).

\subsubsection{Effects of a modulated input signal}

Optimization of a conventional DBD for maximizing the time-averaged force and the resulting electric wind is an important aspect for flow control perspectives. However, like observed with most of the devices designed for manipulating bounded or free flows, plasma actuators driven by burst modulation of the AC carrier signal is most of the time more effective for improving the control performances (Moreau 2007; Corke et al. 2009, 2010). In this part, a description of the electric wind velocity is proposed for DBD supplied by different modulated high-voltage waveforms.

Typical examples of time-averaged velocity profiles of the produced electric wind when the discharge is supplied by a burst modulation at $f_{\mathrm{BM}}$ are shown in Fig. 45 . The duty-cycle of the $f_{\mathrm{BM}}$ square modulating signal is equal to $50 \%$. Results indicate that the shape of the velocity profiles (Fig. 45a) and the spatial location of the maximal velocity (Fig. 45b) remain similar for modulated and not modulated input signal (this suggests that the plasma extension is not affected by the burst modulation). However, the maximum time-averaged velocity is considerably reduced when burst modulation is applied. In the absence of modulation, the maximum velocity is equal to $3 \mathrm{~m} / \mathrm{s}$. When the high voltage is modulated, whatever the $f_{\mathrm{BM}}$ value, the electric wind velocity is reduced down to $2.2 \mathrm{~m} / \mathrm{s}$. In Fig. 46, the effect of the duty-cycle is investigated. It highlights that the mean electric wind velocity and the consumed electrical power increase linearly with the duty-cycle of the burst modulation. This behavior can be characterized by time-resolved measurements of the plasma-induced flow. Figure 47 presents the time history of the electric wind velocity when the discharge is switched on and off with four different duty-cycle values (from 30 to $90 \%$ ). One can observe that 

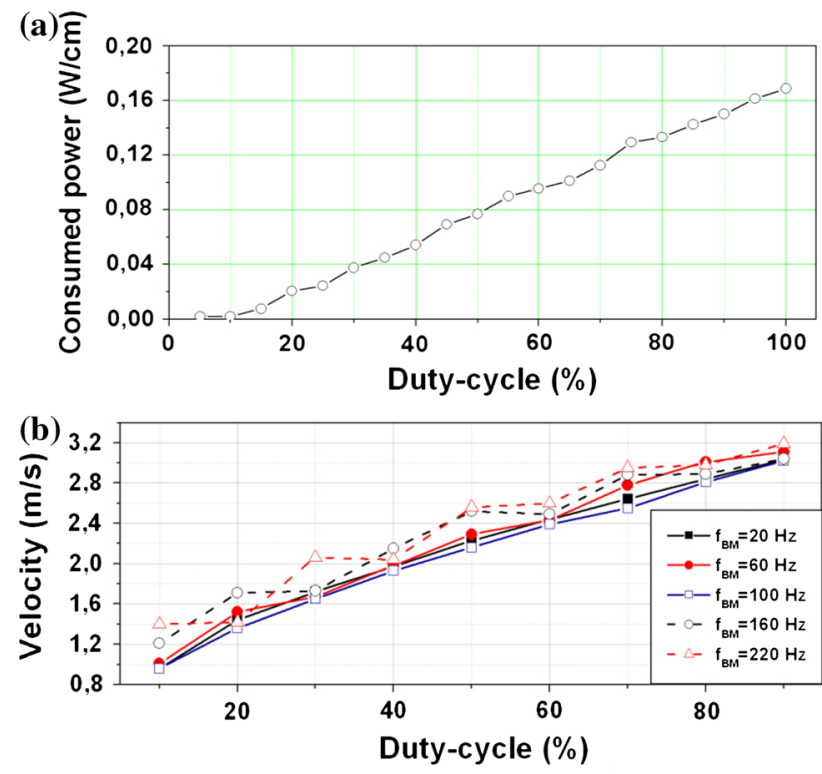

Fig. 46 Illustration of a consumed electrical power and $\mathbf{b}$ induced velocity for actuator operated at $f_{\mathrm{AC}}=1 \mathrm{kHz}$ and $18 \mathrm{kV}$. From Benard and Moreau (2009)

about ten $\mathrm{AC}$ cycles, corresponding to $10 \mathrm{~ms}$, are required to reach the maximal velocity and a steady state regime. When the relaxation time (i.e., the time when the discharge is switched off) is too short, the flow is still moving and then a smaller number of $\mathrm{AC}$ cycles are required to reach the final maximal velocity (Fig. 47d). The off period of the plasma (relaxation period for the surrounding gas) is a preponderant parameter. It determines the time-averaged electric wind velocity, but also dictates the amplitude of the velocity fluctuations imposed by the plasma discharge. However, a change in the burst frequency does not affect the formation process of the electric wind and its mean velocity (Fig. 47b) (i.e., the produced flow velocity is not dependent to the applied frequency for burst actuation mode, in the frequency range investigated here). The main difference concerns the amplitude of the electric wind oscillations. Indeed, at high burst frequency (and constant $f_{\mathrm{AC}}$ ), the number of AC cycles per period is reduced as well as the off period duration. It results that the initial velocity at the beginning of a new on period is higher, and then, despite a lower number of AC cycles, the final mean flow is similar to the one observed at lower burst frequency.

Finally, two timescales coexist in the produced electric wind: the electric wind oscillations at $f_{\mathrm{AC}}$ and larger amplitudes velocity fluctuations at $f_{\mathrm{BM}}$. Under no circumstance, fluctuations at the frequency $f_{\mathrm{AC}}$ of the electrical signal can be considered as purely electrical disturbances of the recorded signal as indicated in a few papers. Although limited to the area of the plasma discharge, these fluctuations are directly related to the fast response time of the actuator and they contribute to the turbulent kinetic energy induced by the discharge. Furthermore, both of these frequencies can contribute to flow control mechanisms, this independently or by coupling effects.

Burst modulation is now usual in flow control by plasma actuator. However, other types of modulation can be applied, such as amplitude modulation and superposition, as defined in Benard and Moreau (2010). For an amplitude modulation at $\mathrm{f}_{\mathrm{AM}}$, the electrical signal is composed of two sidebands in frequency domain (at $f_{\mathrm{AC}}-f_{\mathrm{AM}}$ and $f_{\mathrm{AC}}+f_{\mathrm{AM}}$ ). With superposition performed at $\mathrm{f}_{\mathrm{SP}}$, such
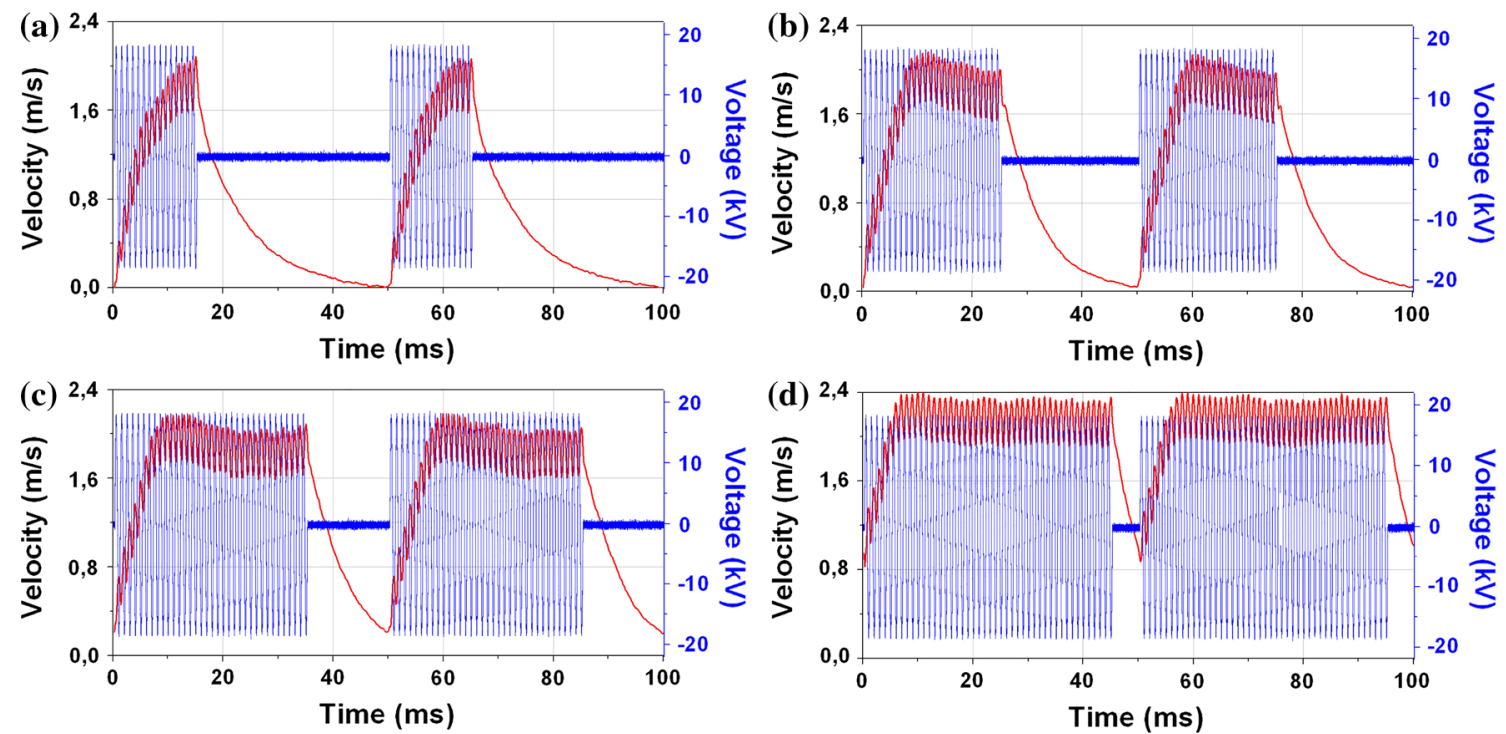

Fig. 47 Phase reconstruction of the electric wind production (LDV measurements) for $V=18 \mathrm{kV}$ at $f_{\mathrm{AC}}=1 \mathrm{kHz}, f_{\mathrm{BM}}=20 \mathrm{~Hz}$ and a $\mathrm{DC}=30 \%, \mathbf{b} \mathrm{DC}=50 \%, \mathbf{c} \mathrm{DC}=70 \%$ and $\mathbf{d} \mathrm{DC}=90 \%$. Voltage in blue and velocity in red. From Benard and Moreau (2009) 

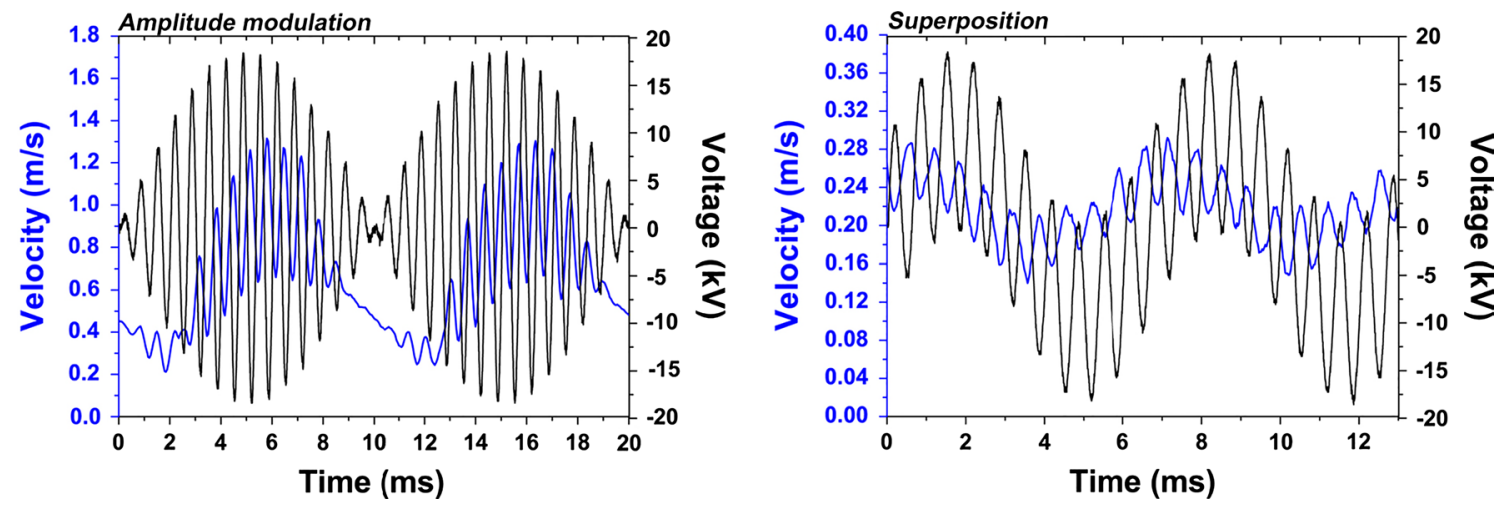

Fig. 48 Phase reconstruction of the time-resolved electric wind production (LDV measurements) for $V=18 \mathrm{kV}$ at $f_{\mathrm{AC}}=1 \mathrm{kHz}$ and modulations by amplitude and superposition (at $f_{\mathrm{AM}}=50 \mathrm{~Hz}$ and $f_{\mathrm{SP}}=150 \mathrm{~Hz}$ ). Voltage in black, velocity in blue. From Benard and Moreau (2010)
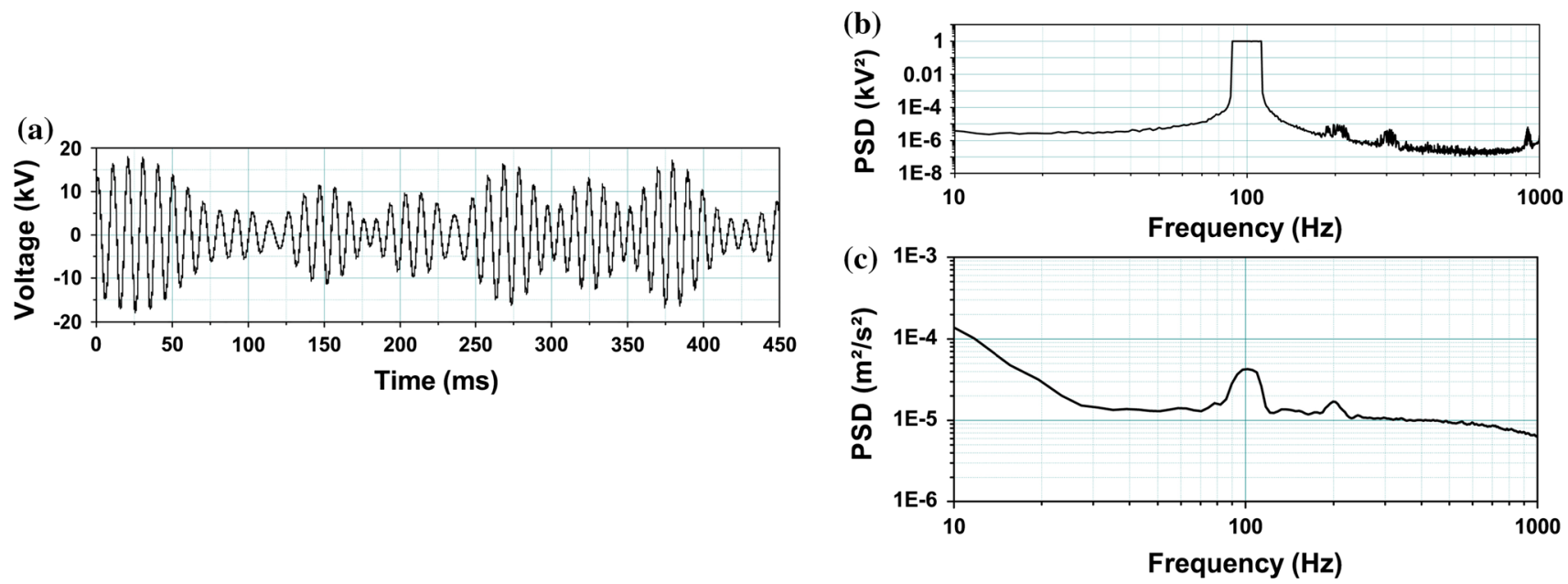

Fig. 49 Tonal excitation by using single DBD (LDV measurements). Views of the electrical signal applied to the actuator (a) and its power spectrum density (b) and power spectrum density of the induced electric wind (c)

modulation produces two sidebands at $f_{\mathrm{SP}}$ and $f_{\mathrm{AC}}$. Figure 48 presents an illustration of the instantaneous local flow field when such types of modulations are applied. As previously observed in the previous section, the produced flow is the mirror of the electrical signal applied to the actuator (with a phase-shift related to the measurement location). Electric wind resulting from superposition and amplitude modulations presents intrinsic properties having potential benefits for flow control. Superposition modulation results in electric wind having two narrowed energy peaks at two frequencies possibly separated by one or several orders of magnitude. However, the fluctuation amplitude remains quite low. This can certainly be improved by adjusting the modulation rate. With amplitude modulation, the flow perturbations are higher and the frequency content is concentrated on two close energy peaks. However, for high modulation rate, the plasma can quench several times over a single $1 / \mathrm{f}_{\mathrm{AM}}$ period. This results in significant flow fluctuations at twice the modulating frequency. This has to be considered when one plans to act with effectiveness on a specific frequency.

In most of the flow control studies, unsteady actuation usually consists in forcing the flow at its most amplified frequency leading to drastic changes in the mean and turbulent component of the manipulated flow. However, flow dynamic is in most cases not strictly periodic, and fluctuations around the preferential frequency are often observed. The fast response time of plasma actuators provides an exceptional opportunity for multi-frequency excitations. Indeed, a single plasma actuator can force the flow 'simultaneously' over a defined range of frequencies by using tonal excitation forcing (Benard and Moreau 2010). An illustration of such modulation is shown in Fig. 49. It consists of predefining a frequency bandwidth composed of a number of frequency peaks (separated by $\Delta f$, see Fig. 49b). The corresponding temporal evolution 
of the electrical signal can be reconstructed by using inverse fast Fourier transform (Fig. 49a). In Fig. 49, the signal has a bandwidth restricted between 90 and $110 \mathrm{~Hz}$ and is composed of 20 discrete frequency peaks (every $1 \mathrm{~Hz}$ ). When applied to the actuator, this signal results in an unsteady electric wind measured here by LDV. The power spectrum density of this electric wind is shown in Fig. 49c. This spectrum exhibits a strong frequency signature over a bandwidth strictly similar to the one of the electrical inputs. It is sure that the unique capacity of dielectric barrier discharge for producing momentum transfer at low timescale is essential for flow control applications related to open and recent closed-loop approaches. It is probable that unsteady actuation by adequate modulation can improve the influence of the actuator on the flow field, especially when instability mechanisms are responsible for the uncontrolled flow dynamics.

\subsection{Multi-electrode arrangements}

This section concerns different types of plasma actuators constructed on multi-electrodes arrangements. This comprises the use of single DBD assembled in series and threeelectrode DBDs.

\subsubsection{DBDs in series}

A way to extend the plasma surface and to increase the velocity of the produced electric wind consists of using several DBDs in series.

The extension of the plasma surface by using several DBDs in series is easily intelligible. Such arrangement has been initially proposed in Roth and Sherman (1998) and Roth (2003). By using 26 single DBDs, they generate a plasma sheet over a surface of $270 \times 270 \mathrm{~mm}^{2}$. By velocity profiles measured with a pressure probe, they demonstrate a maximum plasma-induced velocity up to $4 \mathrm{~m} / \mathrm{s}$. In Roth (2003), they propose a variety of electrode arrangements for DBDs in series, some of them producing normal wall jet when others promote flow acceleration in one or two directions. In order to optimize the produced electric wind, they use a panel of DBDs energized with an 8-phase polyphase power supply. They measure an induced flow of roughly $6 \mathrm{~m} / \mathrm{s}$, and by reversing the electrode phasing, they can produce a reversed traveling wave. In 2000 , the capacity of plasma actuator to produce wall tangential flow in opposite direction without geometrical change in the actuator design was detailed further in Corke and Matlis (2000). They show that the alternation of sign in the velocity component promotes high-amplitude velocity fluctuations over an extended surface, and due to the low response time of the electromechanical conversion, fluctuations at a desired frequency is easily achieved. In Borghi et al. (2008), the

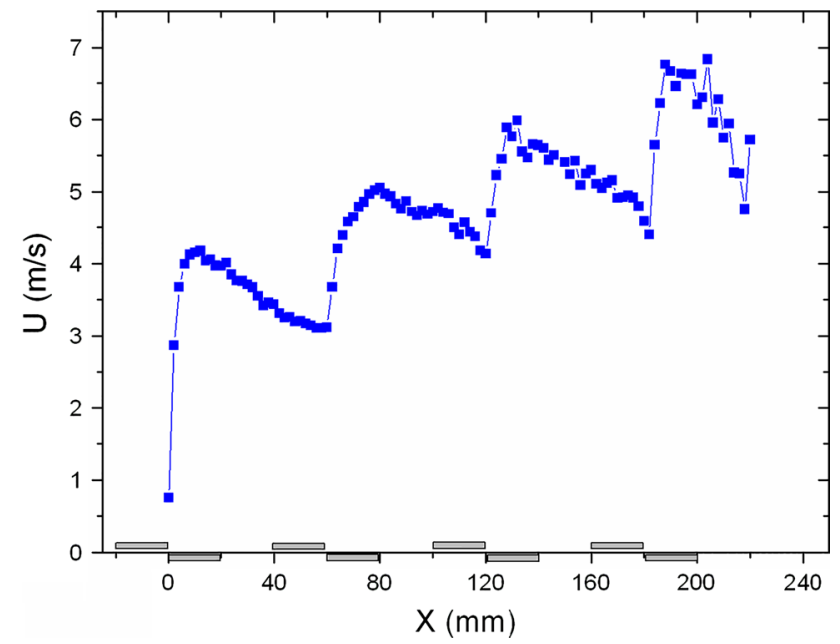

Fig. 50 Horizontal velocity profile $(y=0.5 \mathrm{~mm})$ above four DBD actuators in series. From Forte et al. (2007)

authors investigated the electric wind produced by a panel of 20 electrode pairs. In a first experiment, the panel is supplied by an AC voltage, this voltage being simultaneously applied to each of the active electrodes. They measured a velocity production reaching a maximal value of $4.5 \mathrm{~m} / \mathrm{s}$ by increasing the $\mathrm{AC}$ frequency up to $9 \mathrm{kHz}$. They also investigated a panel made of 9 electrode pairs connected to a three-phase system. However, the gas flow induced by the plasma was not documented in the absence of freestream velocity. In all of these studies, the primary objective was the definition of demonstrators for producing plasma-flow interactions over a large surface, but no systematic measurements were provided regarding the benefits for increasing the produced electric wind.

Dielectric barrier discharges in series can extend the surface of plasma in contact with the surrounding flow, but, as indicated in Enloe et al. (2004b), the effect of multiple actuators is additive if they are placed in reasonably close proximity. For a series of two single DBD, they observe that for a given voltage, the velocity produced by two DBDs in series is twice as big as the velocity measured with only one single DBD. This was verified at low voltage ( $8 \mathrm{kV}$ for $0.3 \mathrm{~mm}$ dielectric thickness) for maximal velocity reaching about $3.8 \mathrm{~m} / \mathrm{s}$. In 2006, four actuators in series were investigated in Forte et al. (2007). Figure 50 shows a horizontal velocity profile (along the $x$ axis) measured $0.5 \mathrm{~mm}$ above the wall. The first DBD produces a flow of $4 \mathrm{~m} / \mathrm{s}$ and then velocity decreases downstream the plasma extension, down to $3 \mathrm{~m} / \mathrm{s}$. With the second DBD $(x=60 \mathrm{~mm})$, velocity drops up to $5 \mathrm{~m} / \mathrm{s}$. In fact, each actuator adds about $1 \mathrm{~m} / \mathrm{s}$ to the maximum velocity of the upstream actuator, but a noticeable saturation effect is observed for the last DBD. Nevertheless, a maximal velocity of $6.7 \mathrm{~m} / \mathrm{s}$ is measured at the end of the DBD panel 
(gain of $62 \%$ between induced flow by the first and last actuators). More importantly than the achieved maximal velocity amplitude, the authors evidenced the presence of a backward discharge responsible for the production of a counter-electric wind (called cross talk phenomenon in Do et al. 2008) if the successive electrodes are placed too close. This is particularly detrimental at high-voltage amplitude because the back discharge can produce a significant electric wind in the opposite direction. This back flow is at the origin of the saturation observed in panels of DBDs, restricting the conclusions of Enloe et al. (2004b) to a limited range of voltage. For instance, with higher voltage amplitudes, it was demonstrated in Thomas et al. (2009) that the thrust does not simply increase linearly with the number of actuators. Indeed, Fig. 51 presents the thrust versus voltage for 1, 2 and 3 single DBDs in series, reaching the maximum value that has been reported up to now with three DBDs. The gain is significant but the thrust gain is lower than $300 \%$.

All of these studies bring a solid background to design more effective multi-DBD actuators. The goal of the recent works on DBDs in series was to cancel the mutual interactions between the air-exposed electrodes of each individual DBDs. In Benard et al. (2009), the approach consists of replacing each single two-electrode DBD by a three-electrode DBD where the third electrode acts as a shield between two successive single DBDs (see Fig. 52). The shield electrode enables to cancel the recirculation zone occurring upstream each air-exposed electrode by reducing the backward discharge. By using this arrangement, the counter-flow electric wind was strongly limited and the flow velocity was maintained at roughly constant amplitude all along the panel. However, the produced flow velocity does not exceed the one reported in Forte et al. (2007). Berendt et al. (2011a) construct a complex multi-DBD actuator based on the combination of smooth high-voltage electrodes, saw-like grounded electrodes and saw-like floating inter-electrodes, made of a 50- $\mu \mathrm{m}$ thick copper tape (Fig. 53a). They conducted PIV measurements in order to characterize the velocity and the topology of the produced flow. Figure 53b presents two velocity profiles at $8 \mathrm{~mm}$ downstream the last high-voltage electrode. Results show that the maximum attained airflow velocity is 8.9 and $10.1 \mathrm{~m} / \mathrm{s}$ at a frequency 1.5 and $2.35 \mathrm{kHz}$, respectively. Another multi-DBD design was simultaneously proposed in Debien et al. (2012a) and Moreau et al. (2013). In this original design, two points have been optimized compared to usual multiDBD. First, the plate air-exposed active electrodes have been replaced by thin wires (diameter of $25 \mu \mathrm{m}$ ) in order to enhance the electric wind produced by each single DBD. Secondly, interaction between successive DBD has been cancelled by alternating the HV electrode and

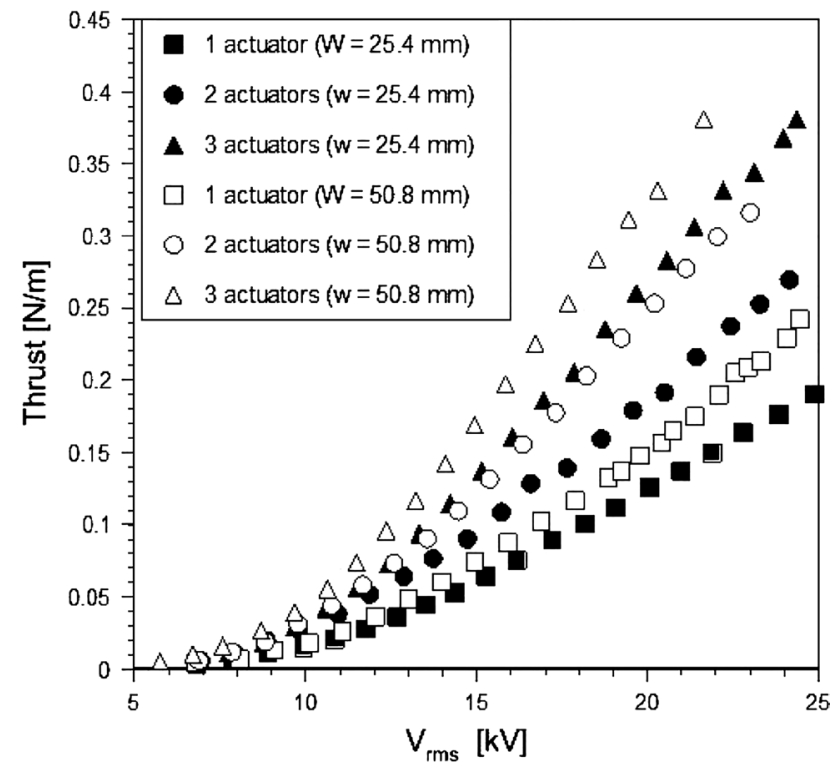

Fig. 51 Actuator thrust for DBD actuators in series. From Thomas et al. (2009)

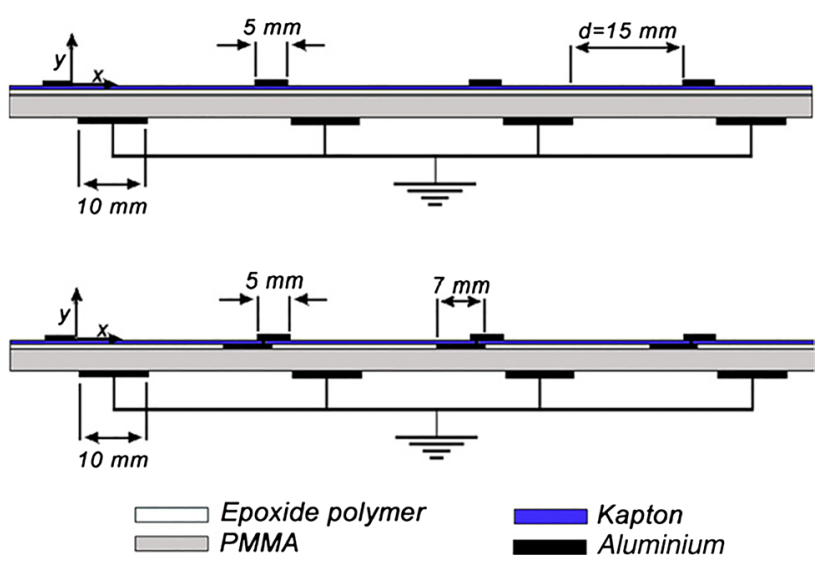

Fig. 52 Electrode arrangement for (top) conventional DBDs in series and (bottom) DBDs with shield electrode (Benard et al. 2009)

the grounded one, from one DBD to the successive one (Fig. 54a). In such conditions, velocity measurements have been conducted with a pressure probe. For instance, Fig. 54b presents horizontal velocity profiles measured $0.6 \mathrm{~mm}$ above the wall, from $x=0$ (position of the first wire HV electrode) to $x=145 \mathrm{~mm}$ ( $25 \mathrm{~mm}$ downstream the right edge of the last grounded electrode) for three different voltages. From $x=0$ to about $15 \mathrm{~mm}$, the velocity increases from zero to $6 \mathrm{~m} / \mathrm{s}$ for $24 \mathrm{kV}$ and then decreases because there is no EHD force downstream the plasma extension. Then, each successive single DBD adds velocity that cumulates up to $10.5 \mathrm{~m} / \mathrm{s}$ downstream the last single DBD, this in the absence of a complete optimization of the actuator design. 

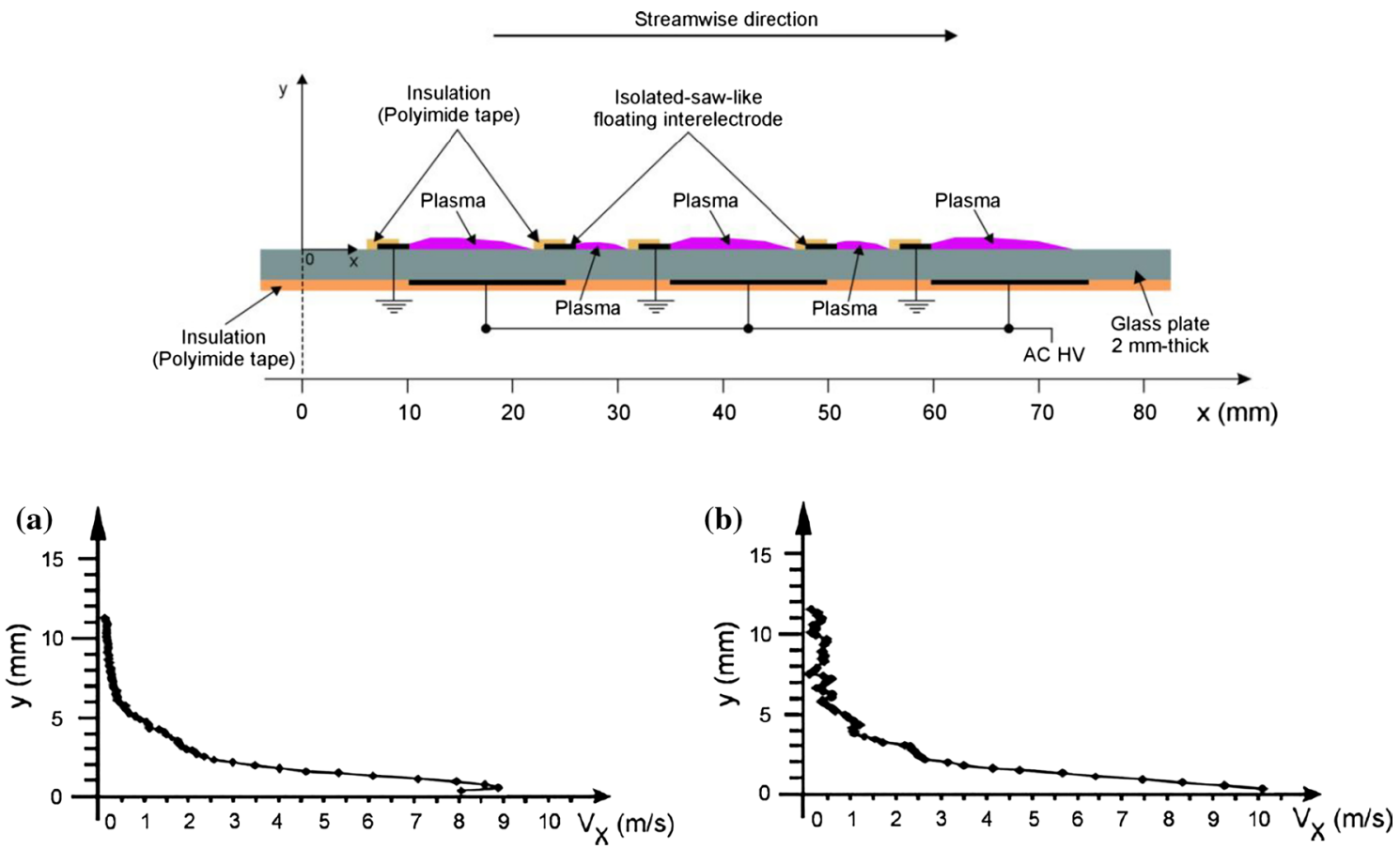

Fig. 53 Sketch of the multi-DBD actuator with isolated saw-like floating inter-electrodes and velocity profiles for AC frequencies of 1.5 and $2.35 \mathrm{kHz}$, while the voltage amplitude is fixed at $16 \mathrm{kV}$. From Berendt et al. (2011a)

Fig. 54 Horizontal velocity profiles measured $0.6 \mathrm{~mm}$ above an inversed multi-DBD actuator. From Moreau et al. (2013)
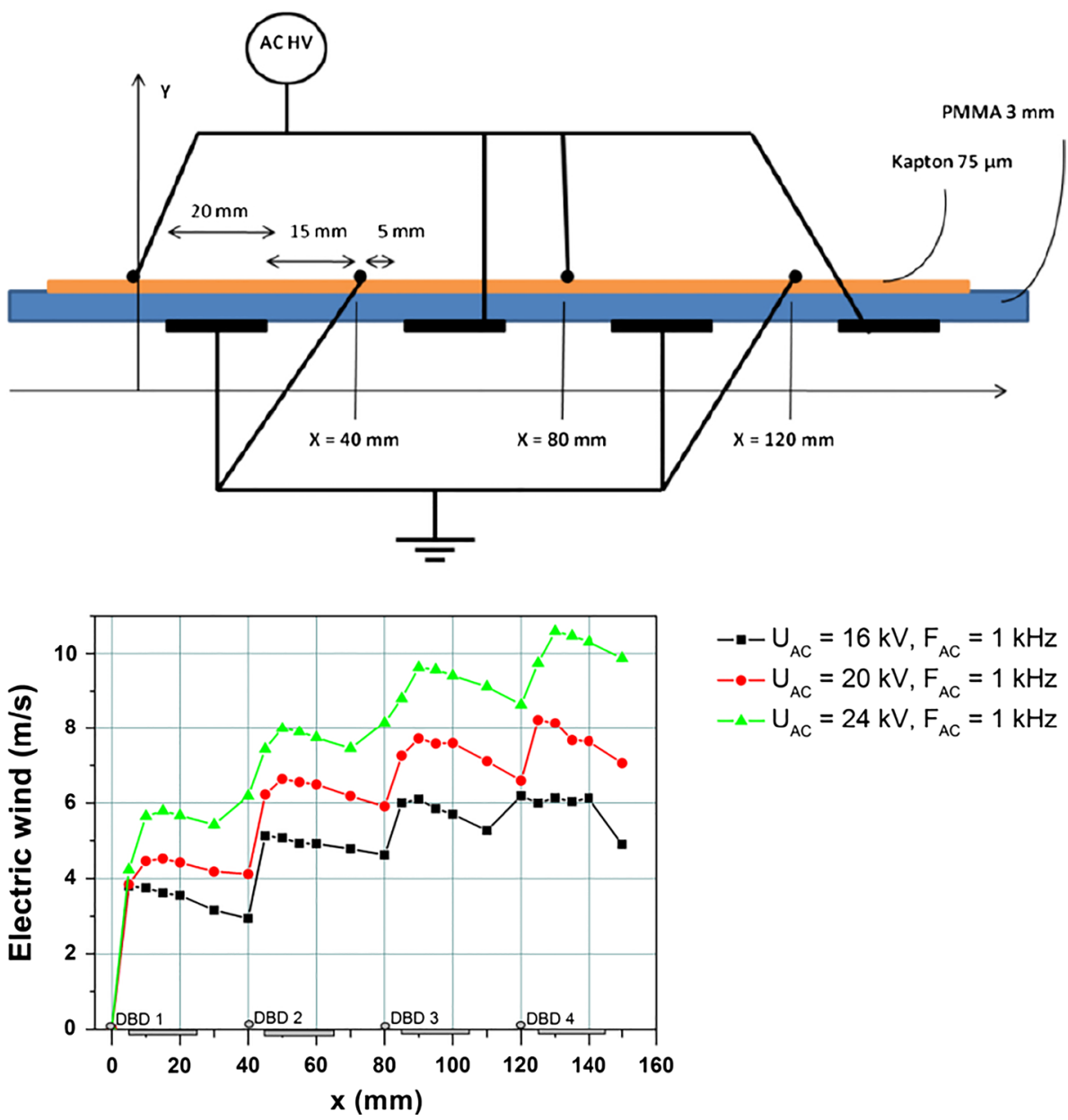
Fig. 55 Sketch of historical three-electrode arrangement

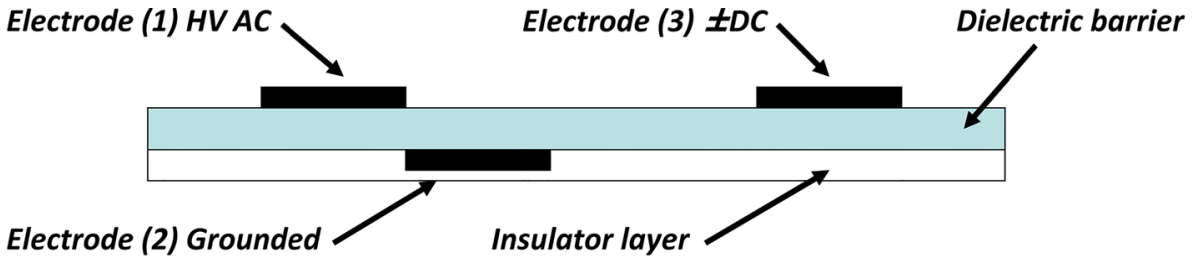

Using DBDs in series for improving the induced gas flow velocity is clearly demonstrated even if parametric optimization was not conducted. In Likhanskii et al. (2010), theoretical limitations of the maximum DBD-induced flow velocity were calculated. For multi-DBDs, they conclude that, when steady state condition is reached, the maximum gas velocity for three DBDs in series is $11.6 \mathrm{~m} / \mathrm{s}$. The recent experimental investigations show that the obtained maximal velocities are close to this theoretical limitation.

\subsubsection{Three-electrode DBD plasma actuators}

Initially, plasma discharges produced by three-electrode arrangement had been designed for homogeneous preionization of species over a large cross section or volume (Nakamura et al. 1986). This work was based on earlier investigation performed by Baranov et al. (1981) for improvement of rare-gas emission. Such discharge was also used for producing cylindrical shock waves in low-pressure environment (Arad et al. 1987). Later, similar electrode arrangement has been used as ion source in pure gas for direct laser pumping (Tsikrikas and Serafetinides 1996) or preionizer in gas laser (Taylor and Leopold 1996). In 2006, a pioneer work investigated the possibility of using an innovative sliding discharge plasma actuator for airflow control applications (Louste et al. 2005) and later in Moreau et al. $(2008 b, c)$. The objective of using sliding discharge as a plasma actuator was to extend the surface of momentum exchanges between the plasma layer and the surrounding flow. This increases the surface of actuation for large-scale applications, and this should enhance the produced force and the resulting electric wind. Several papers have been published on this topic for a few years.

Three-electrode-based system is supplied by an AC high voltage, plus a DC component. It is composed of two electrodes flush mounted on each side of a dielectric such as a single DBD device [electrodes (1) and (2) in Fig. 55], plus a second air-exposed electrode supplied by a DC voltage [electrode (3) in Fig. 55]. Depending on the polarity of the DC voltage $\left(V_{\mathrm{DC}}\right)$, one can produce either a luminescent plasma sheet that occupies the whole electrode gap $\left(V_{\mathrm{DC}}<0\right.$, sliding discharge $)$ or a discharge that visually looks like a $\mathrm{DBD}\left(V_{\mathrm{DC}}>0\right.$, extended $\left.\mathrm{DBD}\right)$. These two types of three-electrode discharges have different electrical and mechanical characteristics that will be shortly described in the following part of the paper. In addition, results concerning the electrode arrangement proposed in Corke et al. (2010) will be discussed.

3.3.2.1 Three electrodes with a third electrode supplied by a positive DC component-extended DBD This configuration, called 'extended DBD,' was perfected for the first time in 2005 by Louste et al. (Louste et al. 2005), and it consists of a three-electrode design where the third electrode is supplied by a positive DC component, as in Fig. 55. Visually, the plasma layer is not different from a conventional single DBD, the visible surface discharge being confined in a region close to the edge of the active electrode (1) supplied by the AC high voltage. Measurements of the discharge current proposed in Louste et al. (2005) show an increase in the capacitive component due to the gas gap between both airexposed electrodes. The authors highlighted that the current was not modified during the positive-going cycle. However, the high negative pulses occur during the negative-going cycle due to the positive DC component bias.

Despite the minor difference in the visual aspect of the discharge, the induced electric wind presents a different topology and amplitude. It had been previously highlighted in Moreau et al. (2008c), and it has been recently verified by PIV measurements in the vicinity of the discharge (Figs. 56, 57 from Debien et al. 2011). The main effect is the enhancement of the suction. In the large region ranged by $-20 \leq x \leq 10 \mathrm{~mm}$ and $0 \leq y \leq 20 \mathrm{~mm}$, the velocity norm is higher than the one measured with a single DBD. Moreover, the suction is reinforced above the discharge $(0 \leq x \leq 40 \mathrm{~mm})$. Extracted from velocity fields, velocity profiles for a single $\mathrm{DBD}\left(V_{\mathrm{AC}}=24 \mathrm{kV}\right)$ and an extended DBD $\left(V_{\mathrm{AC}}=24 \mathrm{kV}\right.$ and $\left.V_{\mathrm{DC}}=24 \mathrm{kV}\right)$ are plotted in Figs. 56 and 57. The velocity profiles measured with the extended DBD are slightly thicker. For instance, for the single DBD, the velocity at $x=30 \mathrm{~mm}$ and $y=10 \mathrm{~mm}$ is close to zero when it is still equal to $0.5 \mathrm{~m} / \mathrm{s}$ for the extended DBD. Moreover, the maximum velocity is lightly higher $(4.45 \mathrm{~m} / \mathrm{s}$ with the extended DBD and $4.25 \mathrm{~m} / \mathrm{s}$ for the single DBD when $V_{\mathrm{AC}}=24 \mathrm{kV}$ ). Indeed, as described in Moreau et al. (2008c), when $V_{\mathrm{DC}}$ is positive, the negative space charge created by the single DBD during the negative-going cycle is more accelerated, resulting in a faster time-averaged velocity. Because the maximum velocity is increased and the electric wind wall jet is thicker with 

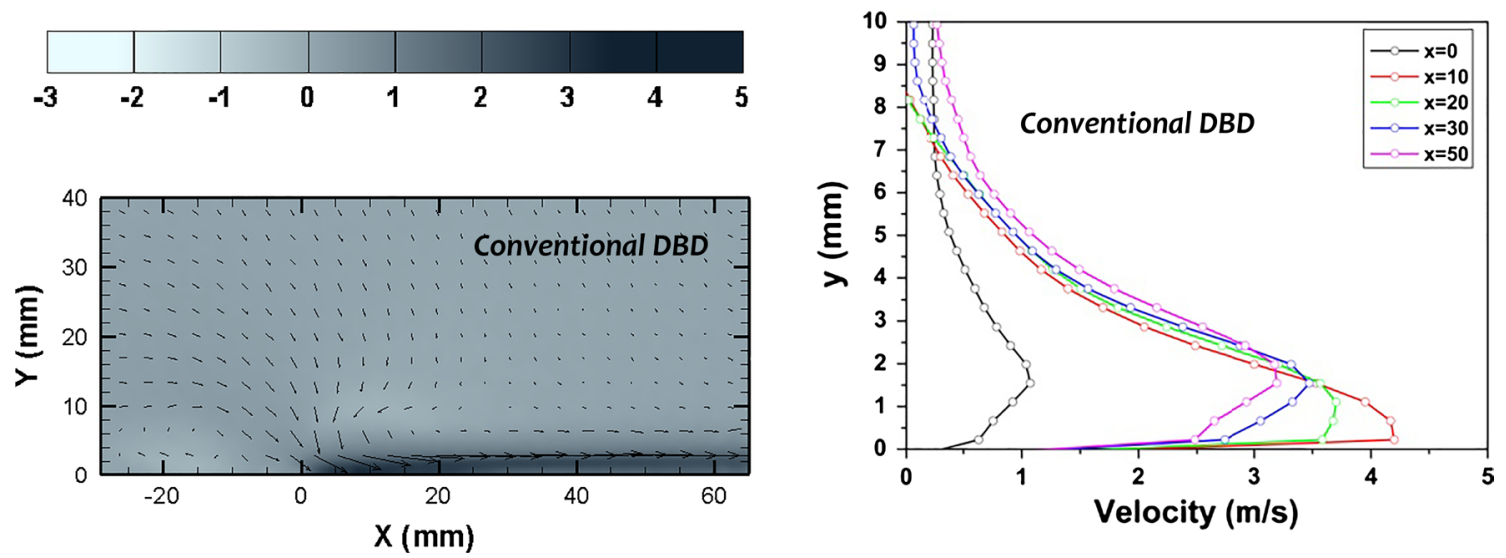

Fig. 56 Flow topology of the electric wind produced by a single DBD $\left(V_{\mathrm{AC}}=20 \mathrm{kV}\right.$ ) and velocity profiles for several $x$ positions (in mm). From Debien et al. (2011)
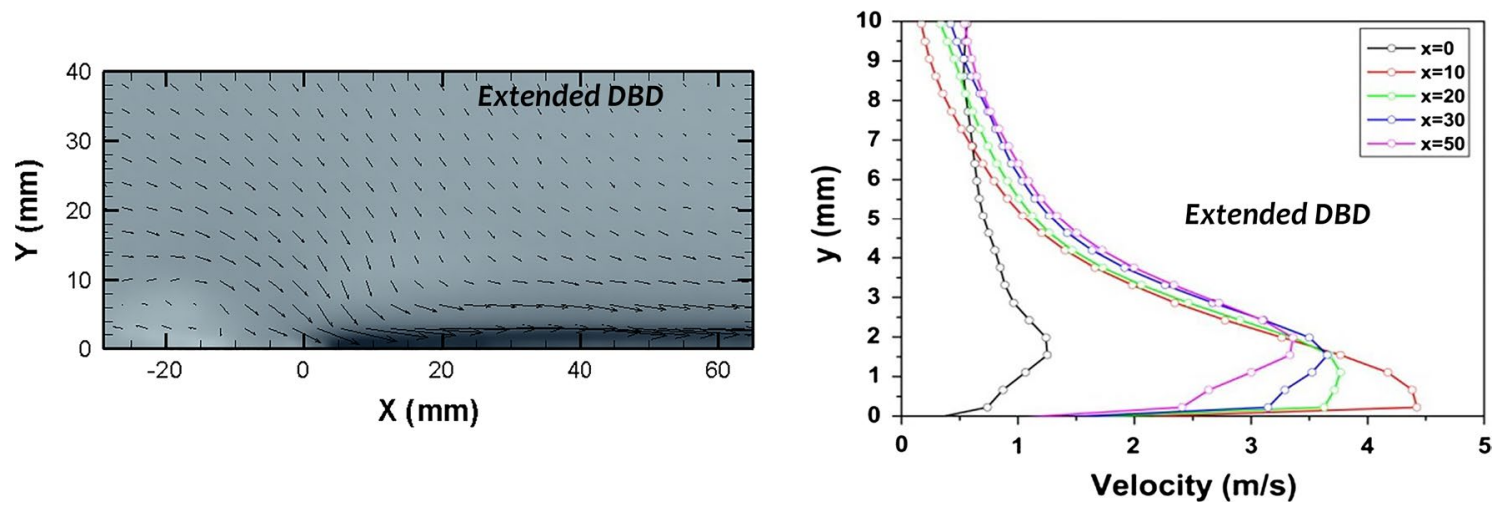

Fig. 57 Flow topology of the electric wind produced by an extended DBD $\left(V_{\mathrm{AC}}=20 \mathrm{kV}, V_{\mathrm{DC}}=16 \mathrm{kV}\right)$ and velocity profiles for several $x$ positions (in mm) $\left(V_{\mathrm{AC}}=24 \mathrm{kV}, V_{\mathrm{DC}}=24 \mathrm{kV}\right)$. From Debien et al. (2011)

$V_{\mathrm{DC}}$, the produced body force should be enhanced. Figure 58 , which presents the discharge thrust for three $V_{\mathrm{AC}}$ values as a function of $V_{\mathrm{DC}}$, shows that the extended DBD significantly increases the produced force, by $45 \%$ for $V_{\mathrm{AC}}=16 \mathrm{kV}$ and only by $10 \%$ when $V_{\mathrm{AC}}=24 \mathrm{kV}$.

To conclude, the DC voltage enhances lightly the mechanical performances of the DBD, but it does not allow for reaching better values than an optimized single DBD.

\subsubsection{Three electrodes with third electrode supplied by a} negative DC component-sliding DBD Initially (Louste et al. 2005; Moreau et al. 2008b; Sosa et al. 2009b), sliding discharges were produced by using the design presented in Fig. 55. In this configuration, it seems that the DBD due to the AC voltage between electrodes (1) and (2) acts as an ionizer. During the positive-going cycle of the sine high voltage (period of positive ion production), electrode (3) that is supplied by a negative DC voltage attracts these positive species. During the negative-going cycle, it seems that the DC component does not modify significantly the surface discharge.

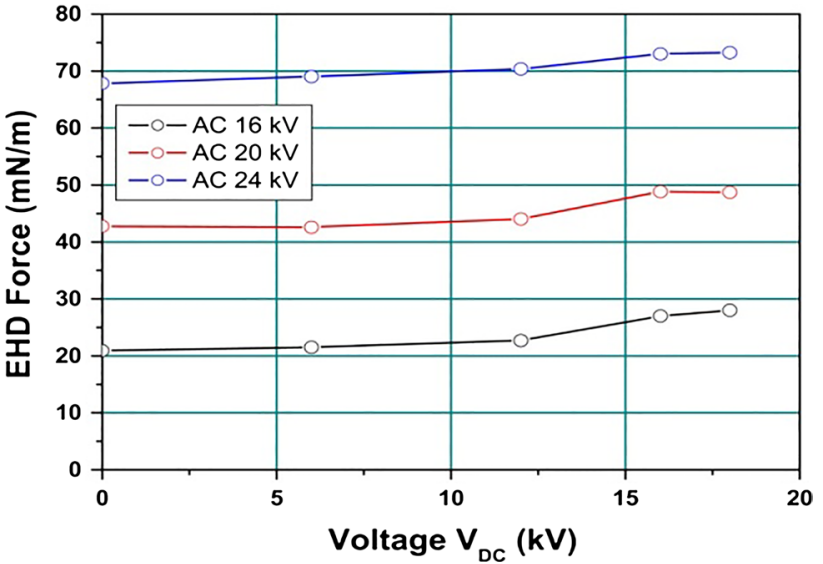

Fig. 58 EHD force induced by an extended DBD versus the DC voltage applied at electrode (3), $V_{\mathrm{DC}}$. From Debien et al. (2011)

The drift of positive species results in generation of a sliding discharge covering the entire gap between both air-exposed electrodes, if the DC voltage is sufficient (above about $6 \mathrm{kV}$ 

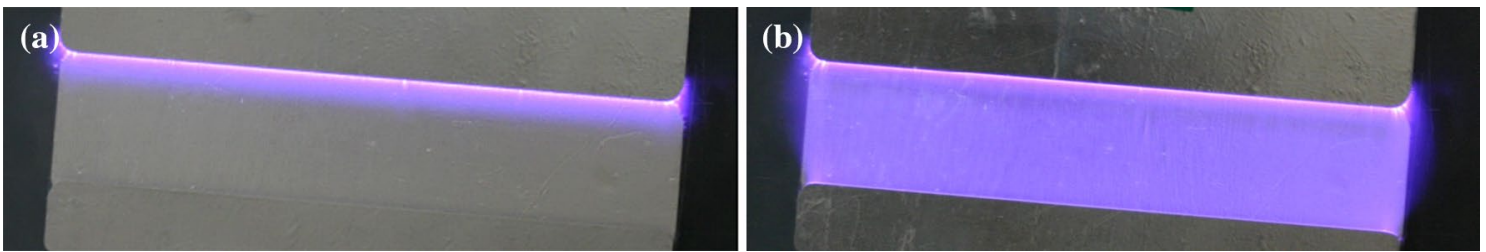

Fig. 59 Top view of a single DBD (a) and a sliding discharge. From Moreau et al. (2008b)

(a)

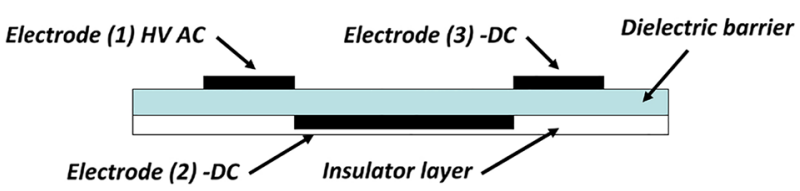

(b) Electrode(1) : - DC Electrode (3): ground

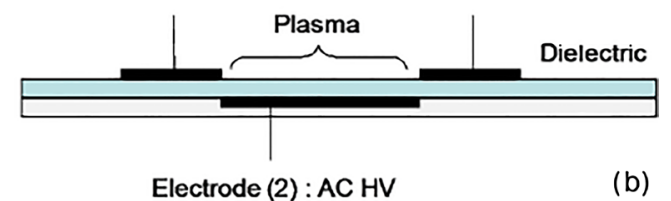

Fig. 60 Complementary designs for producing a sliding discharge

per gap centimeter). An illustration of a sliding discharge is shown in Fig. 59 where the plasma extension due to the negative voltage at electrode (3) is clearly visible. In the absence of DC component, the discharge extension from electrode (1) is limited at less than $1 \mathrm{~cm}$. With a DC component, it extends toward electrode (3) i.e., $4 \mathrm{~cm}$ in this case.

Both complementary electrode arrangements shown in Fig. 60 can be used to produce sliding discharges and have been investigated in several papers. The one presented in Fig. 60a was used in Moreau et al. (2008b, c), Sosa et al. (2008, 2009b), Takashima et al. (2007). The only difference with the design of Fig. 58 is the width of electrode (2). The configuration of Fig. 60b has been initially proposed by Corke et al. (2010). This configuration is very different because the electrodes are not supplied by the same manner; the AC voltage is applied at the encapsulated electrode (2). In the absence of negative DC voltage, two single DBD are produced: one at electrode (1) and the other one at electrode (3). In such conditions, these both single DBDs should produce two opposite horizontal body forces that should form a normal wall jet. Surprisingly, thrust measurements conducted by Corke et al. highlight a high (up to $200 \mathrm{mN} / \mathrm{m}$ ) positive horizontal body force, from electrode (1) to electrode (3), even in the absence of any DC voltage (Fig. 61).

In order to better understand the discharge presented in Fig. 60b, two recent publications have investigated the flow produced by this three-electrode DBD (Debien et al. 2011; Seney et al. 2011). Debien et al. (2011) conducted PIV measurements in order to characterize the flow produced by combination of both discharges occurring at electrodes (1) and (3), for different DC voltage values. In the absence of DC component, a jet perpendicular to the flow

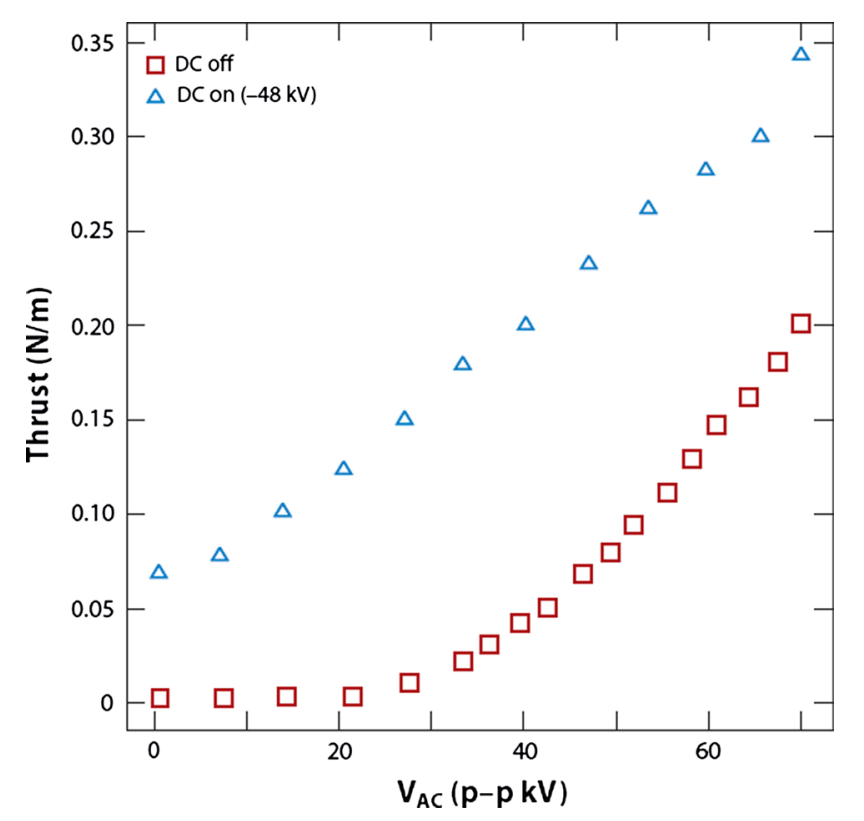

Fig. 61 Thrust versus the applied voltage at electrode (2) in Fig. 60b with and without DC component on electrode (1). From Corke et al. (2010)

is produced. It is due to the interaction between both electric winds: the first electric wind due to the DBD between electrodes (1) and (2) and the second electric wind resulting from the DBD established between electrodes (3) and (2). Both horizontal electric winds have the same intensity with opposite directions. When they collide, a vertical jet is formed. By increasing the negative DC component, the angle of the jet decreases (Fig. 61a, $V_{\mathrm{DC}}=-6 \mathrm{kV}$ ). At $V_{\mathrm{DC}}=-16 \mathrm{kV}$ (Fig. 62b), the presence of the electric wind 

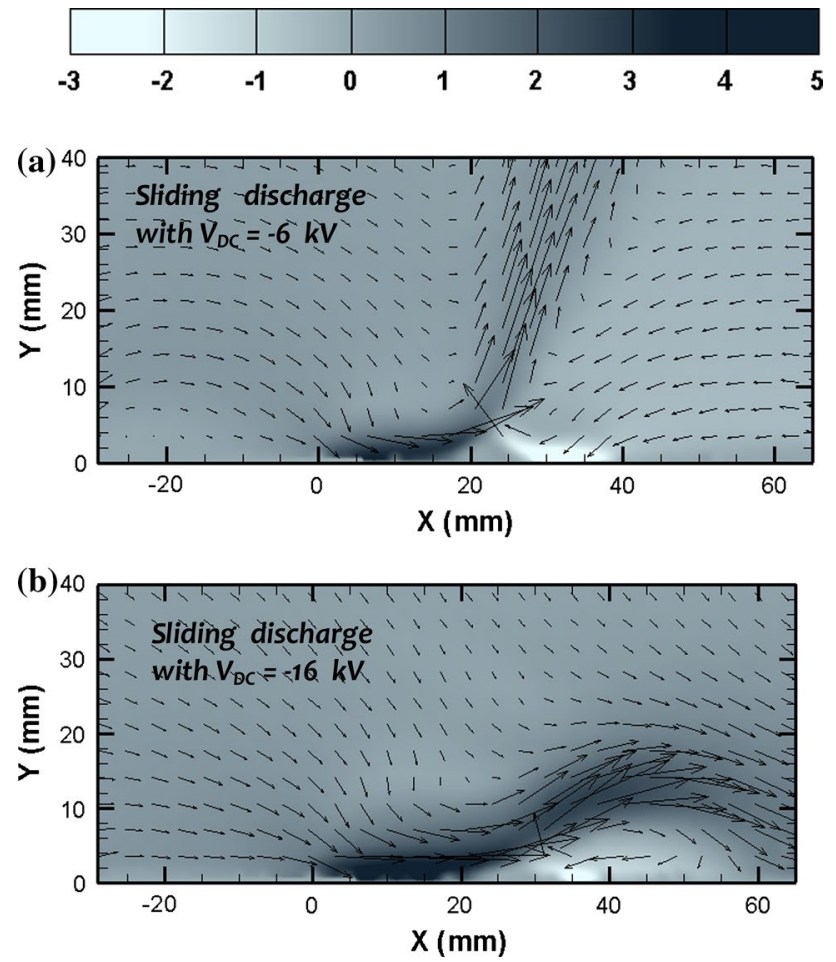

Fig. 62 Flow topology in $\mathrm{m} / \mathrm{s}$ with $V_{\mathrm{AC}}=20 \mathrm{kV}$ and $V_{\mathrm{DC}}=-6 \mathrm{kV}$ and $-16 \mathrm{kV}$ for electrode arrangement shown in Fig. 60b. From Debien et al. (2011)

coming from electrode (3) results in a recirculation zone located at proximity of the edge of electrode (3). In this case, the jet formed from electrode (1) partially detaches from the wall. However, the amplitude of the electric wind occurring at electrode (3) can be reduced by increasing $V_{\mathrm{DC}}$. This is illustrated in Fig. 63 where $V_{\mathrm{AC}}=24 \mathrm{kV}$ and $V_{\mathrm{DC}}=24 \mathrm{kV}$. The flow topology indicates a large region of flow suction above the discharge, a significant thickening of the induced wall jet and an increase in the maximal
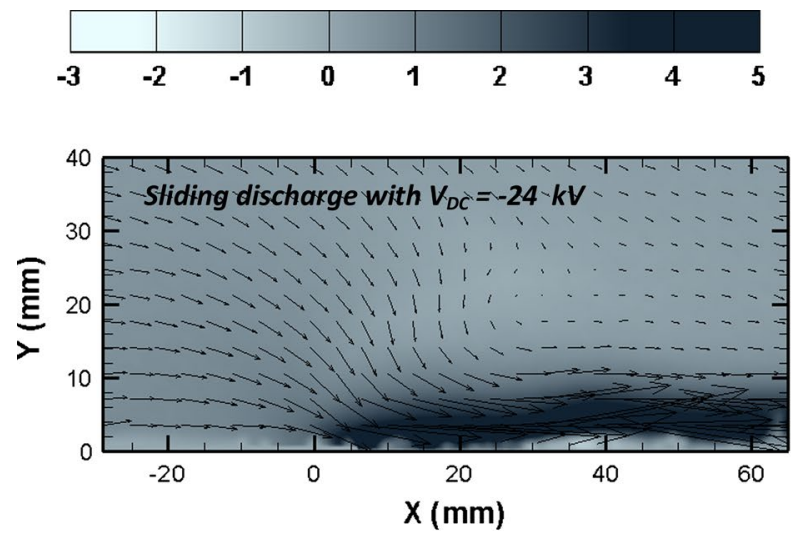

velocity. This can be verified with the velocity profiles proposed in Fig. 63. The produced flow is very different to the one measured with a single DBD and an extended DBD. The wall jet reaches a velocity of $5.5 \mathrm{~m} / \mathrm{s}$ at $2 \mathrm{~mm}$ above the dielectric wall, and the thickening of the jet is evidenced. Finally, this configuration limits losses at the dielectric wall. Indeed, even if it is weak, the counter-electric wind from electrode (3) promotes a flow detachment of the wall jet coming from electrode (1), inducing a decreasing of skin friction. This results in velocity profiles having maximal velocity at a higher distance above the wall than with a single DBD, even if the main jet can be viewed as a tangential wall jet. On the one hand, this can explain the large improvement in thrust production reported in Corke et al. (2010) for a similar electrode arrangement, when a negative DC voltage is applied. On the other hand, in the absence of DC component, the horizontal component of the produced body force should be equal to zero when its vertical component should be increased.

\section{Conclusion}

In this paper, the electrical and mechanical characteristics of surface linear DBD actuators have been summarized. The discussion has been voluntarily limited to actuators that produce a tangential electric wind wall jet, discarding actuators that result in a normal wall jet, such as plasma vortex generator already extensively presented in Wang et al. (2013).

The main results concerning conventional plate-to-plate single DBD plasma actuator are followings:

1. Analysis of discharge current curves and iCCD visualizations highlights that a streamer discharge occurs during the positive-going cycle when a glow-like dis-

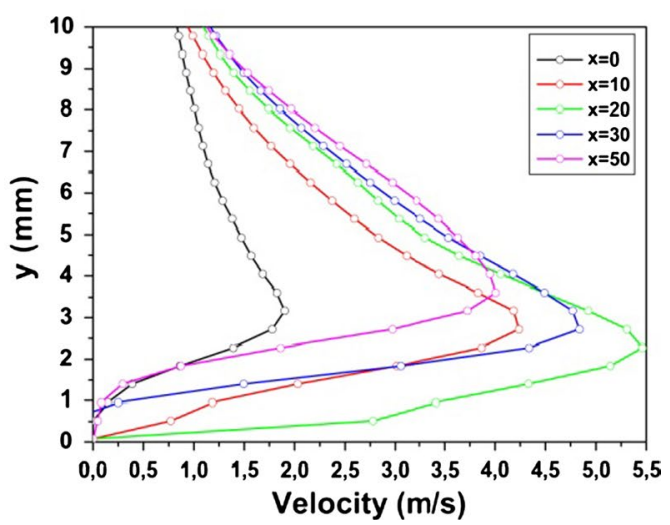

Fig. 63 Flow topology in $\mathrm{m} / \mathrm{s}\left(V_{\mathrm{AC}}=24 \mathrm{kV}, V_{\mathrm{DC}}=-24 \mathrm{kV}\right)$ and velocity profiles for electrode arrangement shown in Fig. 60b. From Debien et al. (2011) 
charge characterized by a quasi-synchronous current ignites during the negative one.

2. Under a voltage threshold, the surface discharge extension increases linearly with the applied high voltage, up to about $20 \mathrm{~mm}$ in the case of a thick dielectric.

3. The typical power consumption ranges from 0.1 to a few $\mathrm{W} / \mathrm{cm}$, with a mean value of $1 \mathrm{~W} / \mathrm{cm}$ for airflow control applications.

4. The maximum measured single discharge thrust is equal to $125 \mathrm{mN} / \mathrm{m}$ with a consumed power of $4 \mathrm{~W} / \mathrm{cm}$.

5. The best effectiveness is equal to $0.6 \mathrm{mN} / \mathrm{W}$ at about $1 \mathrm{~W} / \mathrm{cm}$.

6. In the case of single DBD, the maximum measured electric wind velocity is equal to $7 \mathrm{~m} / \mathrm{s}$, and it is more effective to increase the HV amplitude instead of the $\mathrm{HV}$ frequency to enhance the produced body force and the resulting velocity.

7. Time-resolved velocity measurements highlight that a positive horizontal electric wind is measured for both voltage half-cycles, but the negative-going cycle presents a faster velocity.

8. The high-voltage waveform plays a key role in the time history of the produced electric wind, as the induced flow is the mirror of the applied voltage, showing that the voltage-to-velocity conversion is quasi-linear.

9. The fast response time of discharge provides an exceptional actuator for multi-frequency excitations.

10. At the discharge initiation, a starting vortex is first created and convected by the produced wall flow.

11. The time evolution of the produced EHD force is strongly unsteady with peak values 10 times higher than its time-averaged value. The EHD force seems to be positive during both going cycles of an $\mathrm{AC}$ period, but its amplitude is higher during the negative-going cycle.

12. The local maximum force can reach about $10^{4} \mathrm{~N} / \mathrm{m}^{3}$.

13. The electromechanical efficiency of surface DBD range typically from 0.1 to $0.2 \%$ in quiescent air.

14. In a second part of the paper, it has been shown that the mechanical performances of DBD actuators could be enhanced in optimizing several parameters:

15. The use of a thin wire active electrode allows us to increase the force effectiveness up to $1 \mathrm{mN} / \mathrm{W}$ because both half-cycles produce a fluid acceleration.

16. Multi-electrode arrangements of DBDs in series increase the electric wind velocity, up to about $11 \mathrm{~m} / \mathrm{s}$ in the case of wire-to-plate reversed electrodes.

17. The highest force measured with a multi-DBD actuator is up to $350 \mathrm{mN} / \mathrm{W}$.

18. Sliding discharge can produce large-scale surface plasma actuators, with a slight electric wind enhancement.
Finally, in the case of a single DBD, the best design consists in using a thick dielectric (a few $\mathrm{mm}$ ) at a voltage frequency around $1 \mathrm{kHz}$. With a thick dielectric, the plasma extension is enhanced, the total body force is higher, the resulting electric wind is increased and the actuator is more robust. Furthermore, it has been shown that the spatial and temporal characteristics of the produced electric wind could be optimized for each targeted flow application by modifying the actuator design and by adjusting the highvoltage waveform. Indeed, it is well known that an effective actuation must take place at the right location, at the right moment and at the right frequency in order to interact efficiently with the baseline incoming flow.

Open Access This article is distributed under the terms of the Creative Commons Attribution License which permits any use, distribution, and reproduction in any medium, provided the original author(s) and the source are credited.

\section{References}

Abe T, Takizawa Y, Sato S (2007) A parametric experimental study for momentum transfer by plasma actuator. AIAA paper 2007-0187

Adamovich I, Little J, Nishihara M, Takashima K, Samimy M (2012) Nanosecond pulse surface discharges for high-speed flow control. AIAA Paper 2012-3137

Arad B, Gazit Y, Ludmirsky A (1987) À sliding discharge device for producing cylindrical shock waves. J Phys D Appl Phys 20:360

Ashpis DE, Laun MC, Griebeleer EL (2012) Progress toward accurate measurements of power consumptions of DBD plasma actuators. AIAA paper 2012-0823

Balcon N, Benard N, Moreau E (2009a) Formation process of the electric wind produced by a plasma actuator. IEEE Trans Dielectr Electr Insul 16:140-145

Balcon N, Benard N, Lagmich Y, Boeuf JP, Touchard G, Moreau E (2009b) Positive and negative sawtooth signals applied to a DBD plasma actuator-influence on the electric wind. J Electrost 67:140-145

Baranov VY, Borisov VM, Khristoforov OB (1981) Excimer electricdischarge laser with plasma electrodes. Sov J Quantum Electron 11:93-94

Benard N, Moreau E (2009) Electric wind produced by a surface plasma discharge energized by a burst modulated high voltage. In: Proceedings of the 29th international conference on plasma ionized gazes, ICPIG, 12-17 July, 2009, Cancun

Benard N, Moreau E (2010) Capabilities of the dielectric barrier discharge plasma actuator for multi-frequency excitations. J Phys D Appl Phys 43:145201

Benard N, Moreau E (2011) On the vortex dynamic of airflow reattachment forced by a single non-thermal plasma discharge actuator. Flow Turbul Combust 87:1-37

Benard N, Moreau E (2012) Role of the electric waveform supplying a dielectric barrier discharge plasma actuator. Appl Phys Lett 100:193503

Benard N, Bonnet JP, Touchard G, Moreau E (2008) Flow control by dielectric barrier discharge actuators: jet mixing enhancement. AIAA J 46:2293-2305

Benard N, Mizuno A, Moreau E (2009) A large-scale multiple dielectric barrier discharge actuator based on an innovative three-electrode design. J Phys D Appl Phys 42:235204 
Benard N, Moreau E, Griffin J, Cattafesta L (2010) Slope seeking for autonomous lift improvement by plasma surface discharge. Exp Fluids 48:791-808

Benard N, Cattafesta L, Moreau E, Griffin J, Bonnet J-P (2011) On the benefits of hysteresis effects for closed-loop separation control using plasma actuation. Phys Fluids 23:083601

Benard N, Debien A, Moreau E (2013) Time-dependent volume force produced by a non-thermal plasma actuator from experimental velocity field. J Phys D Appl Phys 46:245201

Berendt A, Podliński J, Mizeraczyk J (2011a) Elongated DBD with floating interelectrodes for actuators. Eur Phys J Appl Phys 55:13804

Berendt A, Podlinski J, Myzeraczyk J (2011b) Comparison of airflow patterns produced by DBD actuators with smooth and saw-like electrode. J Phys Conf Ser 301(1):012018. doi:10.1088/1742-6596/301/1/012018

Boeuf JP, Lagmich Y, Pitchford LC (2009a) Contribution of positive and negative ions to the elecrtrohydrodynamic force in a dielectric barrier discharge operating in air. J Appl Phys 106:023115

Boeuf JP, Lagmich Y, Pitchford LC (2009b) Contribution of positive and negative ions to the electrohydrodynamic force in a dielectric barrier discharge operating in air. J Appl Phys 106:023115

Borghi A, Carraro MR, Cristofolini A, Neretti G (2008) Electrohydrodynamic interaction induced by a dielectric barrier discharge. $\mathrm{J}$ Appl Phys 103:063304

Corke TC, He C (2004) Plasma flaps and slats: an application of weakly-ionized plasma actuator. AIAA Paper 2004-2127

Corke TC, Matlis E (2000) Phased plasma arrays for unsteady flow control. AIAA paper 2000-2323

Corke TC, Post ML (2005) Overview of plasma flow control: concepts, optimization and applications. AIAA paper 2005-563

Corke TC, Post ML, Orlov DM (2009) Single dielectric barrier discharge plasma enhanced aerodynamics: physics, modeling and applications. Exp Fluids 46:1-26

Corke TC, Enloe CL, Wilkinson SP (2010) Dielectric barrier discharge plasma actuators for flow control. Annu Rev Fluid Mech 42:505-529

Debien A, Benard N, Moreau E (2011) Electric wind produced by sliding discharges. In: Proceeding of 2nd ISNPEDADM new electrical technologies for environment, Nouméa

Debien A, Benard N, Moreau E (2012a) Streamer inhibition for improving force and electric wind produced by DBD actuators. J Phys D Appl Phys 45:215201

Debien A, Benard N, David L, Moreau E (2012b) Unsteady aspect of the electrohydrodynamic force produced by surface dielectric barrier discharge actuators. Appl Phys Lett 100:013901

Debien A, Benard N, David L, Moreau E (2012c) Unsteady aspect of the electrohydrodynamic force produced by surface dielectric barrier discharge actuators. Appl Phys Lett 101:229903

Deng J, Matsuoka S, Kumada A, Hidaka K (2010) The influence of residual charge on surface discharge propagation. J Phys D Appl Phys 43:495203

Do A, Kim W, Cappelli MA, Mungal MG (2008) Cross-talk in multiple dielectric barrier discharge actuators. Appl Phys Lett 92:071504

Dong B, Bauchire JM, Pouvesle JM, Magnier P, Hong D (2008) Experimental study of a DBD surface discharge for the active control of subsonic airflow. J Phys D Appl Phys 41:155201

Durscher R, Roy S (2011) Force measurements techniques and preliminary results using aerogels and ferroelectrics for dielectric barrier discharge actuators. AIAA 2011-3735

Durscher R, Roy S (2012) Three-dimensional Flow measurements induced from serpentine plasma actuators in quiescent air. $\mathrm{J}$ Phys D Appl Phys 45:035205
Enloe CL, McLaughlin T, Van Dyken R, Kachner K, Jumper E, Corke TC (2004a) Mechanisms and responses of a single dielectric barrier plasma actuator: plasma morphology. AIAA J 42:589-594

Enloe CL, McLaughlin TE, VanDyken RD, Kachner KD, Jumper EJ, Corke TC, Post M, Haddad O (2004b) Mechanisms and responses of a single dielectric barrier plasma actuator: geometric effects. AIAA J 42:595-604

Enloe CL, McHarg MG, Font GI, McLaughlin TE (2009) Plasmainduced force and self-induced drag in the dielectric barrier discharge aerodynamic plasma actuator. AIAA 2009-1622

Fine NE, Brickner SJ (2010) Plasma catalysis for enhanced-thrust single dielectric barrier discharge plasma actuators. AIAA J 48:2979-2982

Font GI, Enloe CL, McLaughlin TE, Orlov D (2007) Plasma discharge characteristics and experimentally determined boundary conditions for a plasma actuator. AIAA paper 2007-188

Font GI, Enloe CL, McLaughlin TE (2009) Effect of volumetric momentum addition on the total force production of a plasma actuator. AIAA paper 2009-4285

Forte M (2007) Contrôle d'écoulement par actionneur plasma de type décharge à barrière diélectrique-Application à l'amélioration de la pulvérisation dans les injecteurs de turboréacteurs, $\mathrm{PhD}$ Thesis, Poitiers University

Forte M, Léger L, Pons J, Moreau E, Touchard G (2005) DC and pulse plasma actuator for airflow control: measurement of the instationary ionic wind velocity. J Electrost 63:929

Forte M, Jolibois J, Moreau E, Touchard G, Cazalens M (2006) Optimization of a dielectric barrier discharge actuator by stationary and instationary measurements of the induced flow velocity, application to airflow control. AIAA paper 2006-2863

Forte M, Jolibois J, Pons J, Moreau E, Touchard G, Cazalens M (2007) Optimization of a dielectric barrier discharge actuator by stationary and non-stationary measurements of the induced flow velocity: application to airflow control. Exp Fluids 43:917-928

Giepman R, Kotsonis M (2011) On the mechanical efficiency of dielectric barrier discharge plasma actuators. Appl Phys Lett 98:221504

Grundmann S, Tropea C (2007) Experimental transition delay using glow-discharge plasma actuators. Exp Fluids 42:653-657

Grundmann S, Tropea C (2008) Active cancellation of artificially introduced Tollmien-Schlichting waves using plasma actuators. Exp Fluids 44:795-806

Guo S, Burman D, Poon D, Mamunuru M, Simon T, Ernie D, Kortshagen U (2009) Separation control using DBD plasma actuators: designs for thrust enhancement. AIAA paper 2009-4184

Hanson RE, Lavoie P, Naguib A, Morrison JF (2010) Transient growth instability cancelation by a plasma actuator array. Exp Fluids 49:1339-1348

Hollick M, Arjomandi M, Cazzolato B (2011) An investigation into the sensory application of DBD plasma actuators for pressure measurement. Sens Act A Phys 171:102-108

Hong D, Rabat H, Pu YK, Leroy A (2013) Measurement of the surface charging of a plasma actuator using surface DBD. J Electrost 71:547-550

Hoskinson AR, Hershkowitz N (2010) Differences between dielectric barrier discharge plasma actuators with cylindrical and rectangular exposed electrodes. J Phys D Appl Phys 43:065205

Hoskinson AR, Oksuz L, Hershkowitz N (2008a) Microdischarge propagation and expansion in a surface dielectric barrier discharge. Appl Phys Lett 93:221501

Hoskinson AR, Hershkowitz N, Ashpis DE (2008b) Force measurements of single and double barrier DBD plasma actuators in quiescent air. J Phys D Appl Phys 41:245209 
Hoskinson R, Hcrshkowitz N, Ashpis DE (2009) Force measurements of single and double barrier DBD plasma actuators in quiescent air. AIAA paper 2009-485

Jolibois J, Moreau E (2009) Enhancement of the electromechanical performances of a single dielectric barrier discharge actuator. IEEE Trans Dielectr Electr Insul 16:758-767

Joussot R (2011) Etude, caractérisation et amélioration d'un actionneur plasma: application au contrôle de la transition d'une couche limite de Blasius. $\mathrm{PhD}$ Thesis, University of Orléans, France

Joussot R, Hong D, Weber-Rozenbaum R, Leroy-Chesneau A (2010) Modification of the laminar-to-turbulent transition on a flat plate using DBD plasma actuator. AIAA paper 2010-4708

Joussot R, Leroy A, Weber R, Rabat H, Loyer S, Hong D (2013) Plasma morphology and induced airflow characterization of a DBD actuator with serrated electrode. J Phys D Appl Phys 46:125204

Jukes TN, Choi KS (2009) Flow control around a circular cylinder using pulsed dielectric barrier discharge surface plasma. Phys Fluids 21:084103

Kelley C, Bowles P, Cooney J, He C, Corke TC, Osborne B, Silkey J, Zehnle J (2012) High mach number leading-edge flow separation control using AC DBD plasma actuators. AIAA paper 2012-906

Kotsonis K, Ghaemi S (2011) Forcing mechanisms of dielectric barrier discharge plasma actuators at carrier frequency of $625 \mathrm{~Hz}$. J Appl Phys 110:113301

Kotsonis M, Ghaemi S (2012) Performance improvement of plasma actuators using asymmetric high voltage waveforms. J Phys D Appl Phys 45:045204

Kriegseis J, Schroeter D, Grundmann S, Tropea C (2011) Onlinecharacterization of dielectrical barrier discharge plasma actuators for optimized efficiency of aerodynamical flow control applications. J Phys Conf Ser 301(1):012020. doi:10.1088/1742-6596/301/1/012020

Kriegseis J, Grundmann S, Tropea C (2011b) Power consumption, discharge capacitance and light emission as measures for thrust production of dielectric barrier discharge plasma actuators. J Appl Phys 110:013305

Kriegseis J, Schwarz C, Duchmann A, Grundmann S, Tropea C (2012) PIV-based estimation of DBD plasma-actuator force Terms. AIAA paper 2012-0411

Laurentie JC, Jolibois J, Moreau E (2009) Surface dielectric barrier discharge: effect of encapsulation of the grounded electrode on the electromechanical characteristics of the plasma actuator. J Electrost 67:93-98

Léger L, Moreau E, Touchard G (2001) Control of low velocity airflow along a flat plate with a DC electrical discharge. In: Proceedings of the IEEE-IAS world conference on industrial applications of electrical energy (CD-ROM), Chicago, USA, 30 Septembre-4 October

Léger L, Moreau E, Touchard G (2002) Electrohydrodynamic airflow control along a flat plate by a DC surface corona dischargevelocity profile and wall pressure measurements. AIAA paper 2002-2833

Likhanskii A, Shneider M, Macheret S, Miles R (2008) Modeling of dielectric barrier discharge plasma actuator in air. J Appl Phys 103:053305

Likhanskii AV, Shneider M, Opaits DF, Miles R, Macheret S (2010) Limitations of the DBD effects on the external flow. AIAA paper 2010-470

Little J, Samimy S (2010) High-lift airfoil separation control with dielectric barrier discharge plasma actuation. AIAA J 48:521-537

Lombardi AJ, Bowles P, Corke TC (2012) Closed-loop dynamic stall control using a plasma actuator. AIAA-2012-918

Louste C, Artana G, Moreau E, Touchard G (2005) Sliding discharge in air at atmospheric pressure: electrical properties. J Electrost 63:605
Manley C (1943) The electric characteristics of the ozonator discharge. Trans Electrochem Soc 84:83-96

Masuda S, Washizu M (1979) Ionic charging of very high resistivity spherical particle. J Electrost 6:57-67

Matlis EH, Corke TC, Cameron J, Morris S, Fay P (2008) High-bandwidth plasma sensor suite for high-speed high-enthalpy measurements. AIAA-2008-243

McLaughlin TE, Felker B, Avery JC, Enloe CL (2006) Further experiments in cylinder wake modification with dielectric barrier discharge forcing. AIAA paper 2006-1409

Moreau E (2007) Airflow control by non-thermal plasma actuators. J Phys D Appl Phys 40:605

Moreau E, Touchard G (2008) Enhancing the mechanical efficiency of electric wind in corona discharges. J Electrost 66:36-41

Moreau E, Labergue A, Touchard G (2005) About the kinetic power induced by $\mathrm{AC}$ and $\mathrm{DC}$ discharges. In: Proceedings of the IEEE-CEIDP (Nashville, USA, Oct 2005), pp 490-494

Moreau E, Sosa R, Artana G (2008a) Electric wind produced by surface plasma actuators: a new dielectric barrier discharge based on a three-electrode geometry. J Phys D Appl Phys 41:115205

Moreau E, Sosa R, Artana G (2008b) Electric wind produced by surface plasma actuators: a new dielectric barrier discharge based on a three-electrode geometry. J Phys D Appl Phys 41:115204

Moreau E, Louste C, Touchard G (2008c) Electric wind induced by sliding discharge in air at atmospheric pressure. J Electrost 66:107-114

Moreau E, Debien A, Benard N, Jukes T, Whalley R, Choi K-S, Berendt A, Podliński J, Mizeraczyk J (2013) Surface dielectric barrier discharge plasma actuators. ERCOFTAC Bulletin 94:5-10

Nakamura K, Yukawa N, Mochizuki T, Horiguchi S, Nakaya T (1986) Optimization of the discharge characteristics of a laser device employing a plasma electrode. Appl Phys Lett 49:1493-1495

Neiswander BW, Matlis EC, Corke TC (2010) Development of a plasma lens for adaptive optics. AIAA-2010-4843

Neumann A, Friedrich J, Czarske J, Kriegseis J, Grundmann S (2013) Determination of the phase-resolved volume force produced by a dielectric barrier discharge plasma actuator. J Phys D Appl Phys 46:042001

Opaits DF (2012) Dielectric barrier discharge plasma actuator for flow control. NASA final report NASA/CR-2012-217655

Opaits DF, Shneider MN, Miles RB, Likhanskii AV, Macheret SO (2008) Surface charge in dielectric barrier discharge plasma actuators. Phys Plasmas 15:073505

Opaits DF, Zaidi S, Shneider MN, Miles RB, Likhanskii AV, Macheret SO (2009) Improving thrust by suppressing charge build-up in pulsed DBD plasma actuators. AIAA paper 2009-487

Orlov DM (2006) Modelling and simulation of single dielectric barrier discharge plasma actuators. Dissertation, University of Notre Dame, USA

Pons J, Moreau E, Touchard G (2004) Electrical and aerodynamic characteristics of atmospheric pressure barrier discharges inambient air. In: Proceedings of ISNTPT2004 (Floride, USA, 10-14 May, 2004), pp 307-310

Pons J, Moreau E, Touchard G (2005) Asymmetric surface barrier discharge in air at atmospheric pressure: electric properties and induced airflow characteristics. J Phys D Appl Phys 38:3635

Pons J, Moreau E, Touchard G (2007) Electrohydrodynamic properties of surface dielectric barrier discharges in ambient air for aerodynamic airflow control. In: 28th ICPIG, July 15-20, 2007, Prague, Czech Republic

Rethmel C, Little J, Takashima K, Sinha A, Adamovich I, Samimy M (2011) Flow separation control over an airfoil with nanosecond pulse driven DBD plasma actuators. AIAA paper 2011-0487

Roth JR (2003) Aerodynamic flow acceleration using paraelectric and peristaltic electrohydrodynamic effects of a one atmosphere uniform glow discharge plasma. Phys Plasma 10:2117-2126 
Roth JR, Dai X (2006) Optimization of the aerodynamic plasma actuator as an electrohydrodynamic (EHD) electrical device. AIAA paper 2006-1203

Roth JR, Sherman DM (1998) Boundary layer flow control with a one atmosphere uniform glow discharge surface plasma. AIAA Paper 98-0.328

Roth JR, Dai X, Rahel J, and Shermann DM (2005) The physics and phenomenology of paraelectric one atmosphere uniform glow discharge plasma (OAUGDP ${ }^{\mathrm{TM}}$ ) actuators for aerodynamic flow control. AIAA 2005-781

Seney SD, Huffman RE, Bailey W, Lui D, Reeder ME, Stults J (2011) Experimental study on the induced velocity of a three potential sliding discharge DBD actuator. AIAA paper 2011-3732

Sosa R, Kelly H, Grondona D, Marquez A, Lago V, Artana G (2008) Electrical and plasma characteristics of a quasi-steady sliding discharge. J Phys D Appl Phys 41:035202

Sosa R, d'Adamo J, Artana G (2009a) Circular cylinder drag reduction by three-electrode plasma actuators. J Phys: Conf Ser 166:012015

Sosa R, Arnaud E, Memin E, Artana G (2009b) Study of the flow induced by a sliding discharge. IEEE Trans Dielectr Electr Insul $16: 305-311$

Starikovskiy A, Miles R (2013) Dielectric barrier discharge control and flow acceleration enhancement by diode surface. AIAA paper 2013-0754

Takashima K, Zouzou N, Moreau E, Mizuno A, Touchard G (2007) Generation of extended surface barrier discharge on dielectric surface-electrical properties. IJPEST 1:14-20

Takeuchi N, Hamasaki T, Yasuoka K, Sakurai T (2011) Surface charge measurement in surface dielectric barrier discharge by laser polarimetry. J Electrost 69:87-91
Taylor RS, Leopold KE (1996) Surface corona discharge excited rare gas halide lamps. Rev Sci Instrum 67:2100-2106

Thomas FO, Kozlov A, Corke TC (2008) Plasma actuators for cylinder flow control and noise reduction. AIAA J 46:1921-1931

Thomas FO, Corke TC, Iqbal M, Kozlov A, Schatzman D (2009) Optimization of dielectric discharge plasma actuators for active aerodynamic flow control. AIAA J 47:2169-2179

Tsikrikas GN, Serafetinides A (1996) Effect of voltage pulse polarity on the performance of a sliding discharge pumped HF laser. $\mathbf{J}$ Phys D Appl Phys 29:2806

Van Dyken R, McLaughlin TE, Enloe CL (2004) Parametric investigations of a single dielectric barrier plasma actuator. AIAA paper 2004-0846

Wagner HE, Brandenburg R, Kozlov KV, Sonnenfeld A, Michel P, Behnke JF (2003) The barrier discharge: basic properties and applications to surface treatment. Vacuum 71:417-436

Wang JJ, Choi KS, Feng LH, Jukes TN (2013) Recent developments in DBD plasma flow control. Prog Aerosp Sci 62:52-78

Whalley R, Choi KS (2010) Starting, traveling, and colliding vortices: dielectric-barrier-discharge plasma in quiescent air. Phys Fluids 22:091105

Widmann A, Duchmann A, Kurz A, Grundmann S, Tropea C (2012) Measuring Tollmien-Schlichting waves using phase-averaged particle image velocimetry. Exp Fluids. doi:10.1007/ s00348-012-1315-3

Zhu Y, Takada T, Tu D (1995) An optical measurement technique for studying residual surface charge distribution. J Phys D Appl Phys 28:1468 